\title{
Selective Rhodium-Catalyzed C-H Amidation of Azobenzenes with Dioxazolones under Mild Conditions
}

Bomi Jeon, Uiseong Yeon, Jeong-Yu Son, and Phil Ho Lee*

National Creative Research Initiative Center for Catalytic Organic Reactions, Department of Chemistry, Kangwon National University, Chuncheon 24341, Republic of Korea

FAX: (+)-82-33-259-5667. E-mail: phlee@kangwon.ac.kr

Contents

1. General

2. Preparation of substrates

3. General procedure for $\mathrm{Rh}$-catalyzed $\mathrm{C}-\mathrm{H}$ amidation of azobenzenes with dioxazolones ….......... S4

4. General procedure for diamidation of azobenzene with dioxazolones ….............................. S17

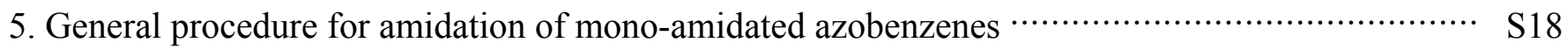

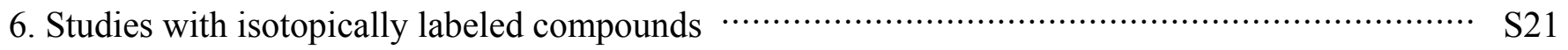

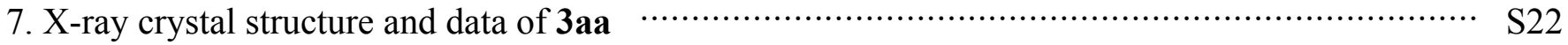

8. References

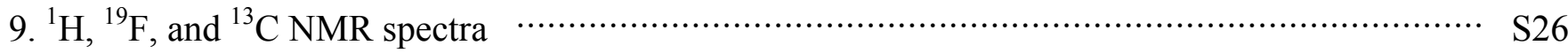




\section{Experimental Section}

1. General: $\left[\mathrm{Cp}^{*} \mathrm{RhCl}_{2}\right]_{2}, \mathrm{AgSbF}_{6}$ and $\mathrm{LiOAc}$ were purchased and were used as received. Commercial available reagents were used without purification. DCE was dried with $\mathrm{CaH}_{2}$. All reaction mixtures were stirred magnetically and were monitored by thin-layer chromatography using silica gel pre-coated glass plates, which were visualized with UV light and then, developed using either iodine or a solution of anisaldehyde. Flash column chromatography was carried out using silica gel (230-400 mesh). ${ }^{1} \mathrm{H}$ NMR (400 MHz), ${ }^{13} \mathrm{C}$ NMR (100 MHz) and ${ }^{19}$ F NMR (376 MHz)spectra were recorded on NMR spectrometer. Deuterated chloroform was used as the solvent, and chemical shift values $(\delta)$ are reported in parts per million relative to the residual signals of this solvent $\left(\delta 7.26\right.$ for ${ }^{1} \mathrm{H}$ and $\delta 77.0$ for ${ }^{13} \mathrm{C}$ ). Infrared spectra were recorded on FT-IR spectrometer as either a thin film pressed between two sodium chloride plates or as a solid suspended in a potassium bromide disk. Mass spectra were obtained from the KBSI on high resolution mass spectrometer. Melting points were determined in open capillary tube using Electrothermal 9100 apparatus. 


\section{Preparation of substrates}

Azobenzenes (1) and 3-substituted 1,4,2-dioxazol-5-ones(2) were prepared by reported method. ${ }^{1,2}$

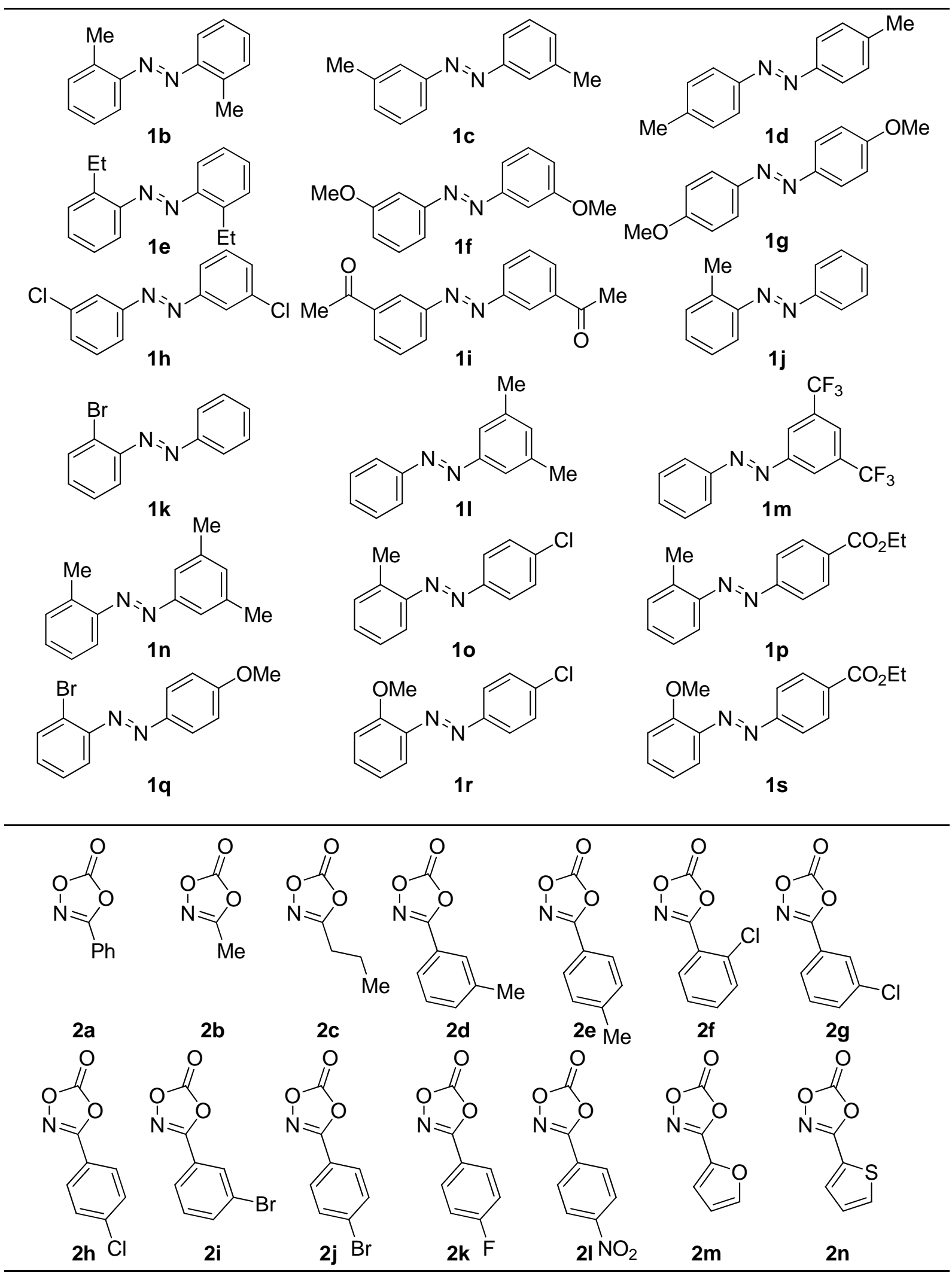


3. General procedure for $\mathrm{Rh}$-catalyzed $\mathrm{C}-\mathrm{H}$ amidation of azobenzenes with dioxazolones

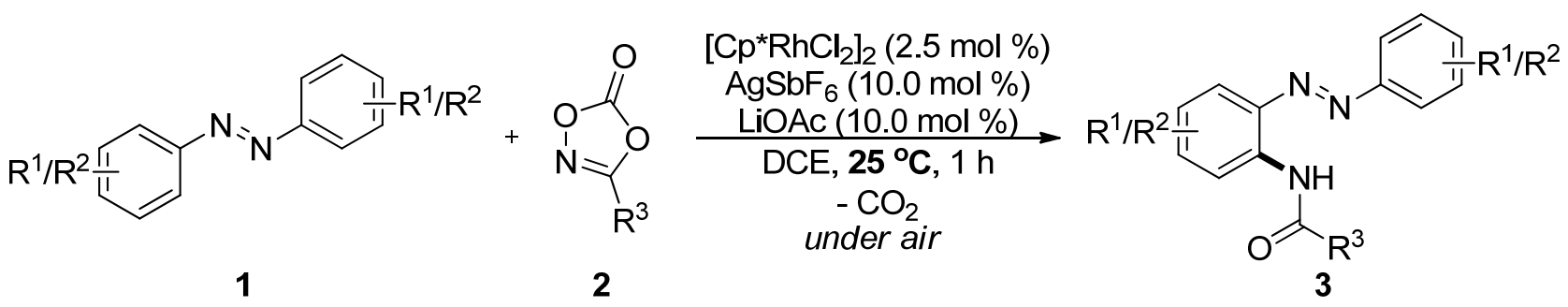

To a test tube were added azobenzene (1) (72.9 mg, $0.4 \mathrm{mmol})$, dioxazolone (2) (32.6 mg, $0.2 \mathrm{mmol})$, $\left[\mathrm{Cp}^{*} \mathrm{RhCl}_{2}\right]_{2}(3.1 \mathrm{mg}, 0.005 \mathrm{mmol}), \mathrm{AgSbF}_{6}(6.9 \mathrm{mg}, 0.02 \mathrm{mmol})$, and LiOAc $(1.3 \mathrm{mg}, 0.02 \mathrm{mmol}) \mathrm{in}$ DCE $(1.0 \mathrm{~mL})$. The resulting mixture was stirred at $25{ }^{\circ} \mathrm{C}$ for $1 \mathrm{~h}$ under air. After celite filtration and evaporation of the solvent in vacuo, the crude product was purified by column chromatography on silica gel using EtOAc:Hexane = 1:7.

(E)-N-(2-(phenyldiazenyl)phenyl)benzamide (3aa): Yield: $50.6 \mathrm{mg}(84 \%) ; R_{f}=0.5$ (EtOAc:Hexane $=$ 1:7); Orange solid; Melting point: $126-128{ }^{\circ} \mathrm{C}$; ${ }^{1} \mathrm{H}$ NMR (400 MHz, $\left.\mathrm{CDCl}_{3}\right) \delta 11.49$ (br, $1 \mathrm{H}$ ), 8.90 (dd, $J$ $=8.4,1.2 \mathrm{~Hz}, 1 \mathrm{H}), 8.01-7.96(\mathrm{~m}, 3 \mathrm{H}), 7.90-7.87(\mathrm{~m}, 2 \mathrm{H}), 7.62-7.48(\mathrm{~m}, 7 \mathrm{H}), 7.25(\mathrm{td}, J=7.7,1.3 \mathrm{~Hz}$, $1 \mathrm{H}) ;{ }^{13} \mathrm{C} \mathrm{NMR}\left(100 \mathrm{MHz}, \mathrm{CDCl}_{3}\right) \delta 165.9,152.3,139.0,135.4,135.1,133.1,132.1,131.4,129.4,128.9$, 127.3, 124.7, 123.6, 122.5, 120.3; IR (film): 3345, 1652, 1592, 1519, 1449, $1316 \mathrm{~cm}^{-1}$; HRMS (EI): $\mathrm{m} / \mathrm{z}$ calcd for $\mathrm{C}_{19} \mathrm{H}_{15} \mathrm{~N}_{3} \mathrm{O}$ : 301.1215; found: 301.1216 .<smiles>O=C(N=Nc1ccccc1)Nc1ccccc1</smiles>

(E)- $N$-(3-methyl-2-(o-tolyldiazenyl)phenyl)benzamide (3ba): Yield: $60.5 \mathrm{mg}$ (92\%); $R_{f}=0.2$ (EtOAc:Hexane = 1:20); Orange solid; Melting point: $110-112{ }^{\circ} \mathrm{C} ;{ }^{1} \mathrm{HNMR}\left(400 \mathrm{MHz}, \mathrm{CDCl}_{3}\right) \delta 12.33$ (br, 1H), $8.71(\mathrm{~d}, J=8.4 \mathrm{~Hz}, 1 \mathrm{H}), 7.86(\mathrm{~d}, J=7.2 \mathrm{~Hz}, 2 \mathrm{H}), 7.56-7.52(\mathrm{~m}, 2 \mathrm{H}), 7.48-7.35(\mathrm{~m}, 4 \mathrm{H}), 7.31(\mathrm{t}$, $J=7.6 \mathrm{~Hz}, 2 \mathrm{H}), 7.15(\mathrm{~d}, J=7.5 \mathrm{~Hz}, 1 \mathrm{H}), 2.74(\mathrm{~s}, 3 \mathrm{H}), 2.36(\mathrm{~s}, 3 \mathrm{H}) ;{ }^{13} \mathrm{C} \mathrm{NMR}\left(100 \mathrm{MHz}, \mathrm{CDCl}_{3}\right) \delta 167.4$, 151.6, 141.1, 137.3, 135.98, 135.96, 132.7, 131.8, 131.5, 131.4, 130.7, 128.7, 127.7, 126.8, 126.2, 118.8, 116.7, 19.9, 18.0; IR (film): 3380, 3184, 3060, 2924, 1680, 1598, 1483, 1212, $707 \mathrm{~cm}^{-1}$; HRMS (EI): $\mathrm{m} / z$ calcd for $\mathrm{C}_{21} \mathrm{H}_{19} \mathrm{~N}_{3} \mathrm{O}$ : 329.1528 ; found: 329.1526 . 


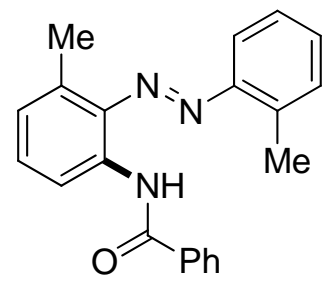

$3 \mathrm{ba}$

(E)- $N$-(4-methyl-2-(m-tolyldiazenyl)phenyl)benzamide (3ca): Yield: $64.4 \mathrm{mg}(98 \%) ; \quad R_{f}=0.2$ (EtOAc:Hexane = 1:20); Orange solid; Melting point: 90-92 ${ }^{\circ} \mathrm{C} ;{ }^{1} \mathrm{H} \mathrm{NMR}\left(400 \mathrm{MHz}, \mathrm{CDCl}_{3}\right) \delta 11.40$ (br, $1 \mathrm{H}), 8.78(\mathrm{~d}, J=8.5 \mathrm{~Hz}, 1 \mathrm{H}), 8.02-7.99(\mathrm{~m}, 2 \mathrm{H}), 7.77(\mathrm{~d}, J=1.5 \mathrm{~Hz}, 1 \mathrm{H}), 7.71(\mathrm{~s}, 1 \mathrm{H}), 7.70(\mathrm{~d}, J=8.6$ $\mathrm{Hz}, 1 \mathrm{H}), 7.62-7.52(\mathrm{~m}, 3 \mathrm{H}), 7.43(\mathrm{t}, J=7.6 \mathrm{~Hz}, 1 \mathrm{H}), 7.36(\mathrm{~d}, J=8.6 \mathrm{~Hz}, 1 \mathrm{H}), 7.34(\mathrm{t}, J=8.8 \mathrm{~Hz}, 1 \mathrm{H})$, $2.48(\mathrm{~s}, 3 \mathrm{H}), 2.43(\mathrm{~s}, 3 \mathrm{H}) ;{ }^{13} \mathrm{C} \mathrm{NMR}\left(100 \mathrm{MHz}, \mathrm{CDCl}_{3}\right) \delta 165.6,152.4,139.2,138.8,135.4,133.7,133.3$, 132.9, 132.1, 131.9, 129.2, 128.8, 127.2, 124.3, 122.7, 120.1, 120.0, 21.4, 20.8; IR (film): 3390, 2917, 1677, 1591, 1448, 1310, $795 \mathrm{~cm}^{-1}$; HRMS (EI): $\mathrm{m} / z$ calcd for $\mathrm{C}_{21} \mathrm{H}_{19} \mathrm{~N}_{3} \mathrm{O}: 329.1528$; found: 329.1529 .

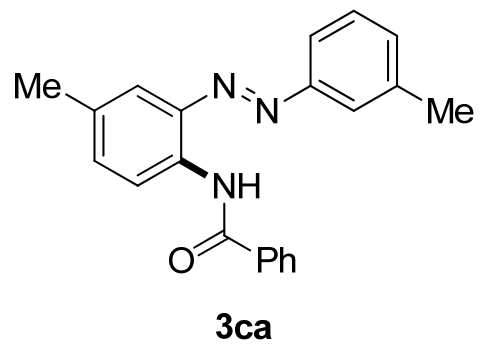

(E)- $N$-(5-methyl-2-(p-tolyldiazenyl)phenyl)benzamide (3da): Yield: $46.0 \mathrm{mg}$ (70\%); $R_{f}=0.4$ (EtOAc:Hexane = 1:7); Yellow solid; Melting point: 198-200 ${ }^{\circ} \mathrm{C} ;{ }^{1} \mathrm{H}$ NMR $\left(400 \mathrm{MHz}, \mathrm{CDCl}_{3}\right) \delta 11.62$ (br, $1 \mathrm{H}), 8.72(\mathrm{~d}, J=0.8 \mathrm{~Hz}, 1 \mathrm{H}), 7.98-7.95(\mathrm{~m}, 2 \mathrm{H}), 7.81(\mathrm{~d}, J=8.1 \mathrm{~Hz}, 1 \mathrm{H}), 7.73(\mathrm{~d}, J=8.3 \mathrm{~Hz}, 2 \mathrm{H}), 7.58-$ $7.49(\mathrm{~m}, 3 \mathrm{H}), 7.29(\mathrm{~d}, J=8.1 \mathrm{~Hz}, 2 \mathrm{H}), 7.02(\mathrm{dd}, J=8.2,1.2 \mathrm{~Hz}, 1 \mathrm{H}), 2.45(\mathrm{~s}, 3 \mathrm{H}), 2.41(\mathrm{~s}, 3 \mathrm{H}) ;{ }^{13} \mathrm{C}$ NMR $\left(100 \mathrm{MHz}, \mathrm{CDCl}_{3}\right) \delta 165.9,150.4,143.8,141.7,137.1,135.4,134.6,132.0,130.0,128.8,127.3,125.3$, 124.6, 122.2, 120.4, 22.2, 21.5; IR (film): 3387, 3029, 2919, 1679, 1603, 1426, 1150, $825 \mathrm{~cm}^{-1}$; HRMS (EI): $m / z$ calcd for $\mathrm{C}_{21} \mathrm{H}_{19} \mathrm{~N}_{3} \mathrm{O}: 329.1528$; found : 329.1525 .<smiles>Cc1ccc(/N=N/c2ccc(C)cc2NC(=O)c2ccccc2)cc1</smiles>

(E)- $N$-(3-ethyl-2-((2-ethylphenyl)diazenyl)phenyl)benzamide (3ea): Yield: $61.4 \mathrm{mg}(86 \%) ; R_{f}=0.3$ (EtOAc: Hexane $=1: 20$ ); Orange solid; Melting point: $72-74{ }^{\circ} \mathrm{C} ;{ }^{1} \mathrm{HNMR}\left(400 \mathrm{MHz}, \mathrm{CDCl}_{3}\right) \delta 12.27$ (br, 
$1 \mathrm{H}), 8.66(\mathrm{dd}, J=8.4,1.3 \mathrm{~Hz}, 1 \mathrm{H}), 7.86-7.83(\mathrm{~m}, 2 \mathrm{H}), 7.56-7.52(\mathrm{~m}, 2 \mathrm{H}), 7.47-7.39(\mathrm{~m}, 4 \mathrm{H}), 7.33-7.29$ $(\mathrm{m}, 2 \mathrm{H}), 7.17(\mathrm{dd}, J=7.5,1.2 \mathrm{~Hz}, 1 \mathrm{H}), 3.17(\mathrm{q}, J=7.5 \mathrm{~Hz}, 2 \mathrm{H}), 2.75(\mathrm{q}, J=7.6 \mathrm{~Hz}, 2 \mathrm{H}), 1.30(\mathrm{t}, J=7.5$ $\mathrm{Hz}, 3 \mathrm{H}), 0.98(\mathrm{t}, J=7.6 \mathrm{~Hz}, 3 \mathrm{H}) ;{ }^{13} \mathrm{C} \mathrm{NMR}\left(100 \mathrm{MHz}, \mathrm{CDCl}_{3}\right) \delta 167.7,151.1,147.4,142.1,136.6,136.1$, $132.8,131.8,131.1,130.9,129.4,128.7,127.7,126.8,124.9,119.0,116.4,26.4,24.5,16.7,15.5$; IR (film): 3378, 3221, 3063, 2968, 1681, 1580, 1455, $1307 \mathrm{~cm}^{-1}$; HRMS (EI): $\mathrm{m} / z$ calcd for $\mathrm{C}_{23} \mathrm{H}_{23} \mathrm{~N}_{3} \mathrm{O}$ : 357.1841; found: 357.1841 .

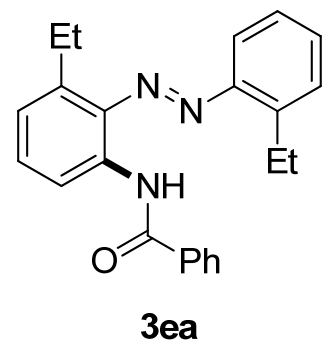

(E)-N-(4-methoxy-2-((3-methoxyphenyl)diazenyl)phenyl)benzamide (3fa): Yield: $41.9 \mathrm{mg}(58 \%) ; R_{f}$ $=0.3$ (EtOAc: Hexane = 1:7); Yellow solid; Melting point: $150-152{ }^{\circ} \mathrm{C} ;{ }^{1} \mathrm{H}$ NMR $\left(400 \mathrm{MHz}, \mathrm{CDCl}_{3}\right) \delta$ 10.88 (br, 1H), 8.81 (d, $J=9.2 \mathrm{~Hz}, 1 \mathrm{H}), 7.98(\mathrm{~d}, J=7.4 \mathrm{~Hz}, 2 \mathrm{H}), 7.60-7.42(\mathrm{~m}, 7 \mathrm{H}), 7.15(\mathrm{dd}, J=9.2,2.8$ $\mathrm{Hz}, 1 \mathrm{H}), 7.08(\mathrm{dt}, J=8.2,1.2 \mathrm{~Hz}, 1 \mathrm{H}), 3.90(\mathrm{~s}, 6 \mathrm{H}) ;{ }^{13} \mathrm{C} \mathrm{NMR}\left(100 \mathrm{MHz}, \mathrm{CDCl}_{3}\right) \delta 165.2,160.5,155.8$, 153.6, 139.8, 135.4, 131.9, 130.2, 130.1, 128.8, 127.1, 121.6, 120.4, 118.4, 117.1, 105.1, 104.9, 55.7, 55.4; IR (film): $3315,2837,1645,1595,1521,1265,1033 \mathrm{~cm}^{-1}$; HRMS (EI): $m / z$ calcd for $\mathrm{C}_{21} \mathrm{H}_{19} \mathrm{~N}_{3} \mathrm{O}_{3}$ : 361.1426; found: 361.1425 .<smiles>COc1cccc(/N=N/c2cc(OC)ccc2NC(=O)c2ccccc2)c1</smiles>

(E)- $N$-(5-methoxy-2-((4-methoxyphenyl)diazenyl)phenyl)benzamide (3ga): Yield: $46.3 \mathrm{mg}(64 \%) ; R_{f}$ $=0.2$ (EtOAc:Hexane = 1:7); Yellow solid; Melting point: $128-130{ }^{\circ} \mathrm{C} ;{ }^{1} \mathrm{H} \mathrm{NMR}\left(400 \mathrm{MHz}, \mathrm{CDCl}_{3}\right) \delta$ $12.06(\mathrm{br}, 1 \mathrm{H}), 8.56(\mathrm{~d}, J=2.7 \mathrm{~Hz}, 1 \mathrm{H}), 8.00(\mathrm{~d}, J=7.1 \mathrm{~Hz}, 2 \mathrm{H}), 7.88(\mathrm{dd}, J=8.9,1.4 \mathrm{~Hz}, 1 \mathrm{H}), 7.82(\mathrm{~d}, J$ $=8.8 \mathrm{~Hz}, 2 \mathrm{H}), 7.63-7.54(\mathrm{~m}, 3 \mathrm{H}), 7.02(\mathrm{~d}, J=8.9 \mathrm{~Hz}, 2 \mathrm{H}), 6.78(\mathrm{dd}, J=8.9,2.7 \mathrm{~Hz}, 1 \mathrm{H}), 3.95(\mathrm{~s}, 3 \mathrm{H})$, $3.90(\mathrm{~s}, 3 \mathrm{H}) ;{ }^{13} \mathrm{C}$ NMR $\left(100 \mathrm{MHz}, \mathrm{CDCl}_{3}\right) \delta 166.3,162.7,161.7,146.5,136.1,135.4,133.6,132.1,128.9$, 128.0, 127.3, 123.7, 114.4, 110.9, 103.5, 55.8, 55.6; IR (film): 3376, 3060, 1676, 1600, 1289, 1254, 836 $\mathrm{cm}^{-1}$; HRMS (EI): $m / z$ calcd for $\mathrm{C}_{21} \mathrm{H}_{19} \mathrm{~N}_{3} \mathrm{O}_{3}: 361.1426$; found: 361.1423 . 


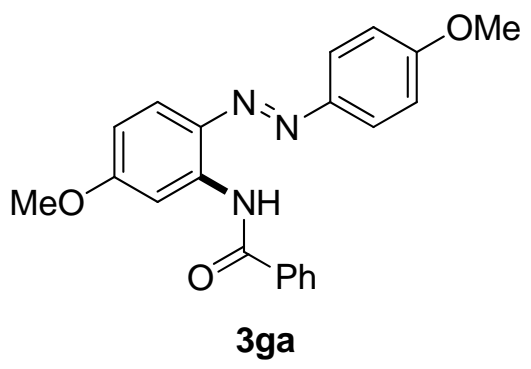

(E)-N-(4-chloro-2-((3-chlorophenyl)diazenyl)phenyl)benzamide (3ha): Yield: $58.4 \mathrm{mg}(79 \%) ; R_{f}=0.5$ (EtOAc:Hexane = 1:7); Yellow solid; Melting point: $162-164{ }^{\circ} \mathrm{C} ;{ }^{1} \mathrm{HNMR}\left(400 \mathrm{MHz}, \mathrm{CDCl}_{3}\right) \delta 10.96$ (br, $1 \mathrm{H}), 8.88(\mathrm{~d}, J=9.0 \mathrm{~Hz}, 1 \mathrm{H}), 7.98-7.96(\mathrm{~m}, 2 \mathrm{H}), 7.93(\mathrm{~d}, J=2.4 \mathrm{~Hz}, 1 \mathrm{H}), 7.87(\mathrm{~s}, 1 \mathrm{H}), 7.84-7.80(\mathrm{~m}, 1 \mathrm{H})$, 7.65-7.51 (m, 6H); $\left.{ }^{13} \mathrm{C} \mathrm{NMR} \mathrm{(100} \mathrm{MHz,} \mathrm{CDCl}_{3}\right) \delta 165.5,152.9,139.3,135.6,134.8,134.7,133.2,132.4$, 131.6, 130.6, 129.1, 129.0, 127.1, 122.5, 122.0, 121.6, 121.3; IR (film): 3327, 1651, 1587, 1515, 1197 , 789, $687 \mathrm{~cm}^{-1}$; HRMS (EI): $\mathrm{m} / z$ calcd for $\mathrm{C}_{19} \mathrm{H}_{13} \mathrm{Cl}_{2} \mathrm{~N}_{3} \mathrm{O}$ : 369.0436 ; found: 369.0433 .<smiles>O=C(Nc1ccc(Cl)cc1/N=N/c1cccc(Cl)c1)c1ccccc1</smiles>

3ha

(E)- $N$-(4-acetyl-2-((3-acetylphenyl)diazenyl)phenyl)benzamide (3ia): Yield: $52.4 \mathrm{mg}(68 \%) ; R_{f}=0.1$ (EtOAc:Hexane = 1:7); Yellow solid; Melting point: 172-174 ${ }^{\circ} \mathrm{C} ;{ }^{1} \mathrm{HNMR}\left(400 \mathrm{MHz}, \mathrm{CDCl}_{3}\right) \delta 11.65$ (br, 1H), $9.04(\mathrm{~d}, J=8.8 \mathrm{~Hz}, 1 \mathrm{H}), 8.61$ (d, $J=2.1 \mathrm{~Hz}, 1 \mathrm{H}), 8.54$ (t, $J=1.8 \mathrm{~Hz}, 1 \mathrm{H}), 8.19-8.12$ (m, 3H), 8.06$8.03(\mathrm{~m}, 2 \mathrm{H}), 7.70(\mathrm{t}, J=7.8 \mathrm{~Hz}, 1 \mathrm{H}), 7.67-7.60(\mathrm{~m}, 3 \mathrm{H}), 2.72(\mathrm{~s}, 3 \mathrm{H}), 2.70(\mathrm{~s}, 3 \mathrm{H}) ;{ }^{13} \mathrm{C} \mathrm{NMR}(100 \mathrm{MHz}$, $\left.\mathrm{CDCl}_{3}\right) \delta 197.0,196.4,166.0,152.1,139.1,138.3,138.0,134.6,133.2,132.7,132.3,131.2,129.9,129.1$, 127.3, 127.1, 125.2, 121.9, 120.1, 26.8, 26.6; IR (film): 3420, 1674, 1586, 1276, 798, $696 \mathrm{~cm}^{-1}$; HRMS (EI): $m / z$ calcd for $\mathrm{C}_{23} \mathrm{H}_{19} \mathrm{~N}_{3} \mathrm{O}_{3}$ : 385.1426; found: 385.1424 .<smiles>O=C(N=Nc1cccc(C(F)(F)F)c1)Nc1ccc(C(F)(F)F)cc1</smiles>

3ia

(E)- $N$-(3-methyl-2-(phenyldiazenyl)phenyl)benzamide (3ja): Yield: $61.2 \mathrm{mg}$ (97\%); $R_{f}=0.5$ (EtOAc:Hexane = 1:10); Orange solid; Melting point: 102-104 ${ }^{\circ} \mathrm{C} ;{ }^{1} \mathrm{HNMR}\left(400 \mathrm{MHz}, \mathrm{CDCl}_{3}\right) \delta 13.13$ (br, 1H), $8.81(\mathrm{~d}, J=8.2 \mathrm{~Hz}, 1 \mathrm{H}), 8.01(\mathrm{~d}, J=6.8 \mathrm{~Hz}, 2 \mathrm{H}), 7.86(\mathrm{~d}, J=7.2 \mathrm{~Hz}, 2 \mathrm{H}), 7.62-7.47$ (m, 6H), 
$7.40(\mathrm{t}, J=8.0 \mathrm{~Hz}, 1 \mathrm{H}), 7.13(\mathrm{~d}, J=7.4 \mathrm{~Hz}, 1 \mathrm{H}), 2.76(\mathrm{~s}, 3 \mathrm{H}) ;{ }^{13} \mathrm{C} \mathrm{NMR}\left(100 \mathrm{MHz}, \mathrm{CDCl}_{3}\right) \delta 167.1$, $152.3,142.5,136.2$, 135.8, 133.2, 132.0, 131.7, 131.1, 129.4, 128.8, 127.6, 125.9, 122.2, 118.5, 19.5; IR (film): 3339, 3062, 2925, 1677, 1582, 1459, 1293, $771 \mathrm{~cm}^{-1}$; HRMS (EI): $\mathrm{m} / z$ calcd for $\mathrm{C}_{20} \mathrm{H}_{17} \mathrm{~N}_{3} \mathrm{O}$ : 315.1372; found: 315.1369 .<smiles>Cc1cccc(NC(=O)c2ccccc2)c1N=Nc1ccccc1</smiles>

3ja

(E)- $N$-(3-bromo-2-(phenyldiazenyl)phenyl)benzamide (3ka): Yield: $57.8 \mathrm{mg} \quad(76 \%) ; \quad R_{f}=0.5$ (EtOAc:Hexane = 1:7); Yellow solid; Melting point: $130-132{ }^{\circ} \mathrm{C} ;{ }^{1} \mathrm{HNMR}\left(400 \mathrm{MHz}, \mathrm{CDCl}_{3}\right) \delta 13.09$ (br, $1 \mathrm{H}), 8.95(\mathrm{dd}, J=8.5,1.2 \mathrm{~Hz}, 1 \mathrm{H}), 8.02-7.99(\mathrm{~m}, 2 \mathrm{H}), 7.97-7.93(\mathrm{~m}, 2 \mathrm{H}), 7.65-7.53(\mathrm{~m}, 7 \mathrm{H}), 7.34(\mathrm{t}, J=$ $8.2 \mathrm{~Hz}, 1 \mathrm{H}) ;{ }^{13} \mathrm{C} \mathrm{NMR}\left(100 \mathrm{MHz}, \mathrm{CDCl}_{3}\right) \delta 167.0,152.1,135.4,134.6,133.4,133.0,132.3,131.9,131.0$, 129.6, 128.9, 128.5, 127.6, 122.8, 120.1; IR (film): 3297, 3062, 1682, 1575, 1515, 1288, 769, $605 \mathrm{~cm}^{-1}$; HRMS (EI): $m / z$ calcd for $\mathrm{C}_{19} \mathrm{H}_{14} \mathrm{BrN}_{3} \mathrm{O}: 379.0320$; found: 379.0323 .<smiles>O=C(Nc1cccc(Br)c1N=Nc1ccccc1)c1ccccc1</smiles>

3ka

(E)- $N$-(2-((3,5-dimethylphenyl)diazenyl)phenyl)benzamide (3la): Yield: $38.2 \mathrm{mg}(58 \%) ; R_{f}=0.4$ (EtOAc:Hexane = 1:7); Orange solid; Melting point: 95-97 ${ }^{\circ} \mathrm{C} ;{ }^{1} \mathrm{HNMR}\left(400 \mathrm{MHz}, \mathrm{CDCl}_{3}\right) \delta 11.62$ (br, $1 \mathrm{H}), 8.90(\mathrm{dd}, J=8.4,1.2 \mathrm{~Hz}, 1 \mathrm{H}), 8.03-8.00(\mathrm{~m}, 2 \mathrm{H}), 7.95(\mathrm{dd}, J=8.0,1.5 \mathrm{~Hz}, 1 \mathrm{H}), 7.63-7.50(\mathrm{~m}, 6 \mathrm{H})$, 7.27-7.23 (m, 1H), 7.16 (br, 1H), $2.43(\mathrm{~s}, 6 \mathrm{H}) ;{ }^{13} \mathrm{C} \mathrm{NMR}\left(100 \mathrm{MHz}, \mathrm{CDCl}_{3}\right) \delta 165.8,152.4,139.1,138.9$, 135.4, 135.1, 133.2, 132.8, 132.1, 128.8, 127.3, 124.7, 123.6, 120.4, 120.2, 21.3; IR (film): 3387, 3060, 2918, 1679, 1595, 1523, 1449, $852 \mathrm{~cm}^{-1}$; HRMS (EI): $\mathrm{m} / z$ calcd for $\mathrm{C}_{21} \mathrm{H}_{19} \mathrm{~N}_{3} \mathrm{O}$ : 329.1528; found: 329.1528. 


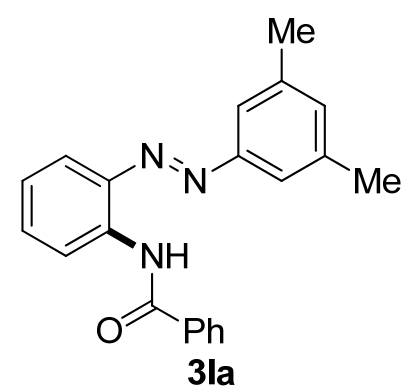

(E)- $N$-(2-((3,5-bis(trifluoromethyl)phenyl)diazenyl)phenyl)benzamide (3ma): Yield: $38.5 \mathrm{mg}$ (44\%); $\mathrm{R}_{f}=0.5$ (EtOAc:Hexane = 1:7); Orange solid; Melting point: $133-135{ }^{\circ} \mathrm{C} ;{ }^{1} \mathrm{H}$ NMR $\left(400 \mathrm{MHz}, \mathrm{CDCl}_{3}\right) \delta$ 11.16 (br, 1H), 8.93 (dd, $J=8.4,2.2 \mathrm{~Hz}, 1 \mathrm{H}), 8.35$ (s, 2H), 8.01-7.97 (m, 4H), 7.64-7.61 (m, 2H), 7.57$7.54(\mathrm{~m}, 2 \mathrm{H}), 7.29-7.25(\mathrm{~m}, 1 \mathrm{H}) ;{ }^{13} \mathrm{C} \mathrm{NMR}\left(100 \mathrm{MHz}, \mathrm{CDCl}_{3}\right) \delta 165.5,152.6,138.6,136.4,135.0,134.9$, $133.1\left(\mathrm{q}, J_{\mathrm{CF}}=34.0 \mathrm{~Hz}\right), 132.5,129.0,127.1,124.1\left(\mathrm{q}, J_{\mathrm{CF}}=3.5 \mathrm{~Hz}\right), 123.8,123.4,122.9\left(\mathrm{q}, J_{\mathrm{CF}}=267.2\right.$ $\mathrm{Hz}), 122.4\left(\mathrm{q}, J_{\mathrm{CF}}=3.7 \mathrm{~Hz}\right), 120.4 ;{ }^{19} \mathrm{~F}$ NMR $\left(376 \mathrm{MHz}, \mathrm{CDCl}_{3}\right) \delta-63.0$; IR (film): 3329, 1653, 1592, 1368, 1278, 1133, $897 \mathrm{~cm}^{-1}$; HRMS (EI): $\mathrm{m} / z$ calcd for $\mathrm{C}_{21} \mathrm{H}_{13} \mathrm{~F}_{6} \mathrm{~N}_{3} \mathrm{O}: 437.0963$; found: 437.0965 .<smiles>O=C(N=Nc1cc(C(F)(F)F)cc(C(F)(F)F)c1)Nc1ccccc1</smiles>

(E)-N-(2-((3,5-dimethylphenyl)diazenyl)-3-methylphenyl)benzamide (3na): Yield: $48.8 \mathrm{mg}(71 \%) ; R_{f}$ $=0.4$ (EtOAc:Hexane = 1:7); Orange solid; Melting point: $118-120{ }^{\circ} \mathrm{C} ;{ }^{1} \mathrm{H}$ NMR $\left(400 \mathrm{MHz}, \mathrm{CDCl}_{3}\right) \delta$ 13.18 (br, 1H), $8.80(\mathrm{dd}, J=8.4,0.5 \mathrm{~Hz}, 1 \mathrm{H}), 8.03-8.00(\mathrm{~m}, 2 \mathrm{H}), 7.62-7.52(\mathrm{~m}, 3 \mathrm{H}), 7.48(\mathrm{br}, 2 \mathrm{H}), 7.38(\mathrm{t}$, $J=8.0 \mathrm{~Hz}, 1 \mathrm{H}), 7.13(\mathrm{~s}, 1 \mathrm{H}), 7.12(\mathrm{~d}, J=7.6 \mathrm{~Hz}, 1 \mathrm{H}), 2.76(\mathrm{~s}, 3 \mathrm{H}), 2.42(\mathrm{~s}, 6 \mathrm{H}) ;{ }^{13} \mathrm{C} \mathrm{NMR}(100 \mathrm{MHz}$, $\left.\mathrm{CDCl}_{3}\right) \delta 167.0,152.4,142.2,139.1,136.3,135.9,132.9,132.8,131.9,131.8,128.6,127.7,125.9,120.1$, 118.5, 21.4, 19.5; IR (film): 3428, 3060, 2920, 1673, 1610, 1584, 1262, $701 \mathrm{~cm}^{-1}$; HRMS (EI): $m / z$ calcd for $\mathrm{C}_{22} \mathrm{H}_{21} \mathrm{~N}_{3} \mathrm{O}$ : 343.1685 ; found: 343.1683 .<smiles>Cc1cc(C)cc(/N=N/c2c(C)cccc2NC(=O)c2ccccc2)c1</smiles> 
(E)- $N$-(2-((4-chlorophenyl)diazenyl)-3-methylphenyl)benzamide (3oa): Yield: $55.9 \mathrm{mg}(80 \%)$; $\mathrm{R}_{f}=$ 0.4 (EtOAc:Hexane = 1:7); Orange solid; Melting point: $148-150{ }^{\circ} \mathrm{C} ;{ }^{1} \mathrm{H}$ NMR $\left(400 \mathrm{MHz}, \mathrm{CDCl}_{3}\right) \delta$ 13.02 (br, 1H), 8.80 (d, $J=8.4 \mathrm{~Hz}, 1 \mathrm{H}), 7.98$ (d, $J=7.1 \mathrm{~Hz}, 2 \mathrm{H}), 7.79$ (d, $J=8.7 \mathrm{~Hz}, 2 \mathrm{H}), 7.63-7.49$ (m, $5 \mathrm{H}), 7.42(\mathrm{t}, J=8.0 \mathrm{~Hz}, 1 \mathrm{H}), 7.14(\mathrm{~d}, J=7.4 \mathrm{~Hz}, 1 \mathrm{H}), 2.74(\mathrm{~s}, 3 \mathrm{H}) ;{ }^{13} \mathrm{C} \mathrm{NMR}\left(100 \mathrm{MHz}, \mathrm{CDCl}_{3}\right) \delta 167.0$, $150.7,142.6$, 137.0, 136.1 135.8, 133.6, 132.1, 131.8, 129.7, 128.8, 127.5, 126.0, 123.3, 118.5, 19.5; IR (film): 3166, 3055, 1675, 1581, 1262, 1085, 784, $706 \mathrm{~cm}^{-1}$; HRMS (EI): $\mathrm{m} / z$ calcd for $\mathrm{C}_{20} \mathrm{H}_{16} \mathrm{ClN}_{3} \mathrm{O}$ : 349.0982; found: 349.0983 .

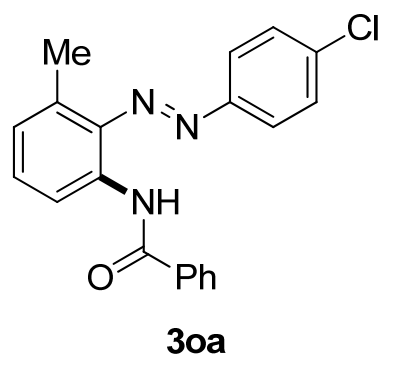

(E)-ethyl 4-((2-benzamido-6-methylphenyl)diazenyl)benzoate (3pa): Yield: $63.5 \mathrm{mg}(82 \%)$; $\mathrm{R}_{f}=0.3$ (EtOAc:Hexane = 1:7); Orange solid; Melting point: $114-116{ }^{\circ} \mathrm{C} ;{ }^{1} \mathrm{H}$ NMR $\left(400 \mathrm{MHz}, \mathrm{CDCl}_{3}\right) \delta 13.05$ (br, 1H), $8.81(\mathrm{~d}, J=8.4 \mathrm{~Hz}, 1 \mathrm{H}), 8.20(\mathrm{~d}, J=8.4 \mathrm{~Hz}, 2 \mathrm{H}), 7.99(\mathrm{~d}, J=7.5 \mathrm{~Hz}, 2 \mathrm{H}), 7.87$ (d, $J=8.4 \mathrm{~Hz}$, 2H), 7.62-7.53 (m, 3H), $7.43(\mathrm{t}, J=8.0 \mathrm{~Hz}, 1 \mathrm{H}), 7.14(\mathrm{~d}, J=7.4 \mathrm{~Hz}, 1 \mathrm{H}), 4.42(\mathrm{q}, J=7.1 \mathrm{~Hz}, 2 \mathrm{H}), 2.76(\mathrm{~s}$, $3 \mathrm{H}), 1.43(\mathrm{t}, J=7.1 \mathrm{~Hz}, 3 \mathrm{H}) ;{ }^{13} \mathrm{C} \mathrm{NMR}\left(100 \mathrm{MHz}, \mathrm{CDCl}_{3}\right) \delta 167.1,165.9,154.7,143.2,136.4,135.7$, $134.2,132.2$, 132.1, 131.8, 130.9, 128.8, 127.6, 126.0, 121.9, 118.5, 61.4, 19.5, 14.3; IR (film): 3162, 3076, 2941, 1733, 1681, 1606, 1587, 1273, $770 \mathrm{~cm}^{-1}$; HRMS (EI): $\mathrm{m} / z$ calcd for $\mathrm{C}_{23} \mathrm{H}_{21} \mathrm{~N}_{3} \mathrm{O}_{3}: 387.1583$; found: 387.1586 .<smiles>CCOC(=O)c1ccc(/N=N/c2c(C)cccc2NC(=O)c2ccccc2)cc1</smiles>

3pa

(E)- $N$-(3-bromo-2-((4-methoxyphenyl)diazenyl)phenyl)benzamide (3qa) : Yield: $60.7 \mathrm{mg}(74 \%) ; R_{f}=$ 0.3 (EtOAc:Hexane = 1:7); Yellow solid; Melting point: 172-174 ${ }^{\circ} \mathrm{C} ;{ }^{1} \mathrm{H}$ NMR $\left(400 \mathrm{MHz}, \mathrm{CDCl}_{3}\right) \delta$ 13.07 (br, 1H), $8.91(\mathrm{dd}, J=8.5,1.1 \mathrm{~Hz}, 1 \mathrm{H}), 7.99-7.97(\mathrm{~m}, 2 \mathrm{H}), 7.94-7.90(\mathrm{~m}, 2 \mathrm{H}), 7.63-7.54(\mathrm{~m}, 4 \mathrm{H})$, $7.28(\mathrm{t}, J=8.2 \mathrm{~Hz}, 1 \mathrm{H}), 7.03(\mathrm{~d}, J=9.0 \mathrm{~Hz}, 2 \mathrm{H}), 3.91(\mathrm{~s}, 3 \mathrm{H}) ;{ }^{13} \mathrm{C} \mathrm{NMR}\left(100 \mathrm{MHz}, \mathrm{CDCl}_{3}\right) \delta 166.9$, $162.8,146.5,135.5,134.6,132.8,132.5,132.2$, 130.1, 128.8, 128.5, 127.6, 124.7, 120.1, 114.7, 55.7; IR (film): 3127, 3063, 1685, 1602, 1578, 1256, 1026, 782, $550 \mathrm{~cm}^{-1}$; HRMS (EI): $\mathrm{m} / z$ calcd for $\mathrm{C}_{20} \mathrm{H}_{16} \mathrm{BrN}_{3} \mathrm{O}_{2}$ : 409.0426; found: 409.0428 . 


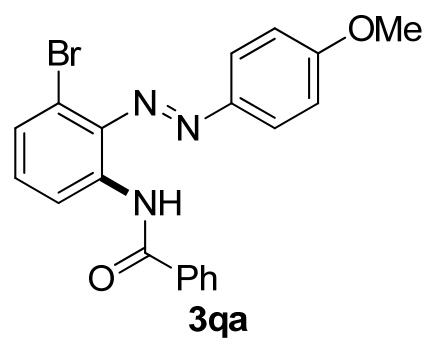

(E)-N-(2-((4-chlorophenyl)diazenyl)-3-methoxyphenyl)benzamide (3ra): Yield: $69.5 \mathrm{mg}(95 \%) ; R_{f}=$ 0.2 (EtOAc:Hexane = 1:7); Orange solid; Melting point: 151-153 ${ }^{\circ} \mathrm{C} ;{ }^{1} \mathrm{H}$ NMR $\left(400 \mathrm{MHz}, \mathrm{CDCl}_{3}\right) \delta$ 13.19 (br, 1H), 8.56 (dd, $J=8.5,1.0 \mathrm{~Hz}, 1 \mathrm{H}), 7.99-7.96(\mathrm{~m}, 2 \mathrm{H}), 7.81-7.78(\mathrm{~m}, 2 \mathrm{H}), 7.63-7.47$ (m, 6H), $6.83(\mathrm{dd}, J=8.3,0.7 \mathrm{~Hz}, 1 \mathrm{H}), 4.02(\mathrm{~s}, 3 \mathrm{H}) ;{ }^{13} \mathrm{C} \mathrm{NMR}\left(100 \mathrm{MHz}, \mathrm{CDCl}_{3}\right) \delta 167.2,159.2,150.9,136.8$, 135.6, 135.0, 132.9, 132.1, 129.6, 128.8, 128.5, 127.6, 123.3, 112.5, 106.6, 56.5; IR (film): 3119, 3062, 1677, 1584, 1275, 1122, $701 \mathrm{~cm}^{-1}$; HRMS (EI): $\mathrm{m} / z$ calcd for $\mathrm{C}_{20} \mathrm{H}_{16} \mathrm{ClN}_{3} \mathrm{O}_{2}$ : 365.0931 ; found: 365.0929 .

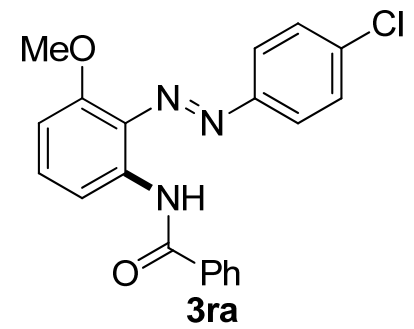

(E)-ethyl 4-((2-benzamido-6-methoxyphenyl)diazenyl)benzoate (3sa): Yield: $60.5 \mathrm{mg}(75 \%) ; R_{f}=0.1$ (EtOAc:Hexane = 1:7); Orange solid; Melting point: $142-143{ }^{\circ} \mathrm{C} ;{ }^{1} \mathrm{HNMR}\left(400 \mathrm{MHz}, \mathrm{CDCl}_{3}\right) \delta 13.27$ (br, $1 \mathrm{H}), 8.58(\mathrm{dd}, J=8.6,1.2 \mathrm{~Hz}, 1 \mathrm{H}), 8.19$ (d, $J=8.4 \mathrm{~Hz}, 2 \mathrm{H}), 8.00(\mathrm{~d}, J=7.2 \mathrm{~Hz}, 2 \mathrm{H}), 7.89$ (dt, $J=8.5$, $1.5 \mathrm{~Hz}, 2 \mathrm{H}), 7.63-7.49(\mathrm{~m}, 4 \mathrm{H}), 6.84(\mathrm{~d}, J=8.3 \mathrm{~Hz}, 1 \mathrm{H}), 4.42$ (q, $J=7.1 \mathrm{~Hz}, 2 \mathrm{H}), 4.04$ (s, 1H), 1.43 (t, $J$ $=7.1 \mathrm{~Hz}, 3 \mathrm{H}) ;{ }^{13} \mathrm{C} \mathrm{NMR}\left(100 \mathrm{MHz}, \mathrm{CDCl}_{3}\right) \delta 167.2,165.9,159.6,155.0,135.6,135.5,133.0,132.2$, 132.0, 130.8, 128.8, 128.7, 127.6, 121.9, 112.4, 106.6, 61.3, 56.6, 14.3; IR (film): 3116, 2982, 1682, 1596, 1481, 1274, 1123, $773 \mathrm{~cm}^{-1}$; HRMS (EI): $\mathrm{m} / z$ calcd for $\mathrm{C}_{23} \mathrm{H}_{21} \mathrm{~N}_{3} \mathrm{O}_{4}$ : 403.1532; found: 403.1535 .<smiles>CCOC(=O)c1ccc(/N=N/c2c(NC(=O)c3ccccc3)cccc2OC)cc1</smiles>

(E)- $N$-(4-methyl-2-(m-tolyldiazenyl)phenyl)acetamide (3cb): Yield: $52.4 \mathrm{mg} \quad(98 \%) ; \quad R_{f}=0.2$ (EtOAc:Hexane = 1:7); Yellow solid; Melting point: $123-125{ }^{\circ} \mathrm{C} ;{ }^{1} \mathrm{HNMR}\left(400 \mathrm{MHz}, \mathrm{CDCl}_{3}\right) \delta 10.02$ (br, $1 \mathrm{H}), 8.55(\mathrm{~d}, J=8.4 \mathrm{~Hz}, 1 \mathrm{H}), 7.66-7.64(\mathrm{~m}, 3 \mathrm{H}), 7.43(\mathrm{t}, J=7.6 \mathrm{~Hz}, 1 \mathrm{H}), 7.32(\mathrm{~d}, J=7.5 \mathrm{~Hz}, 1 \mathrm{H}), 7.29$ $(\mathrm{dd}, J=8.5,1.9 \mathrm{~Hz}, 1 \mathrm{H}), 2.48(\mathrm{~s}, 3 \mathrm{H}), 2.38(\mathrm{~s}, 3 \mathrm{H}), 2.27(\mathrm{~s}, 3 \mathrm{H}) ;{ }^{13} \mathrm{C} \mathrm{NMR}\left(100 \mathrm{MHz}, \mathrm{CDCl}_{3}\right) \delta 168.4$, 
$152.5,139.3$, 138.6, 133.59, 133.56, 133.1, 132.1, 129.1, 123.3, 121.2, 120.1, 119.6, 25.4, 21.5, 20.8; IR (film): 3296, 2918, 1660, 1594, 1309, 1292, 818, $687 \mathrm{~cm}^{-1}$; HRMS (EI): $\mathrm{m} / z$ calcd for $\mathrm{C}_{16} \mathrm{H}_{17} \mathrm{~N}_{3} \mathrm{O}$ : 267.1372; found: 267.1371 .

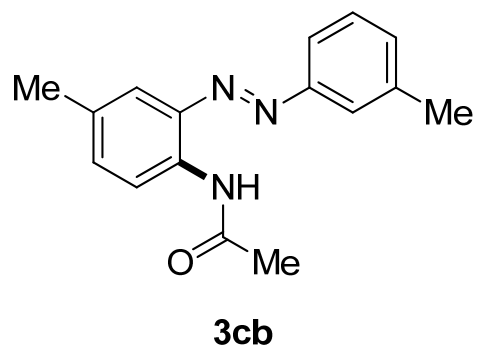

(E)-N-(4-methyl-2-(m-tolyldiazenyl)phenyl)butyramide (3cc): Yield: $43.7 \mathrm{mg}(74 \%) ; R_{f}=0.4$ (EtOAc:Hexane = 1:7); Orange solid; Melting point: 123-125 ${ }^{\circ} \mathrm{C} ;{ }^{1} \mathrm{HNMR}\left(400 \mathrm{MHz}, \mathrm{CDCl}_{3}\right) \delta 10.05$ (br, $1 \mathrm{H}), 8.58(\mathrm{~d}, J=8.5 \mathrm{~Hz}, 1 \mathrm{H}), 7.66-7.64(\mathrm{~m}, 3 \mathrm{H}), 7.43$ (t, $J=7.6 \mathrm{~Hz}, 1 \mathrm{H}), 7.32(\mathrm{~d}, J=7.5 \mathrm{~Hz}, 1 \mathrm{H}), 7.29$ $(\mathrm{dd}, J=8.5,1.9 \mathrm{~Hz}, 1 \mathrm{H}), 2.48(\mathrm{~s}, 3 \mathrm{H}), 2.45(\mathrm{t}, J=7.5 \mathrm{~Hz}, 2 \mathrm{H}), 2.38(\mathrm{~s}, 3 \mathrm{H}), 1.82$ (sextet, $J=7.4 \mathrm{~Hz}, 2 \mathrm{H})$, $1.05(\mathrm{t}, J=7.4 \mathrm{~Hz}, 3 \mathrm{H}) ;{ }^{13} \mathrm{C} \mathrm{NMR}\left(100 \mathrm{MHz}, \mathrm{CDCl}_{3}\right) \delta 171.4,152.6,139.3,138.7,133.64,133.59,133.0$, 132.0, 129.1, 123.2, 121.3, 120.2, 119.7, 40.4, 21.5, 20.8, 19.0, 13.8; IR (film): 3298, 2959, 1656, 1593, 1523, 1251, $687 \mathrm{~cm}^{-1}$; HRMS (EI): $m / z$ calcd for $\mathrm{C}_{18} \mathrm{H}_{21} \mathrm{~N}_{3} \mathrm{O}: 295.1685$; found: 295.1687.

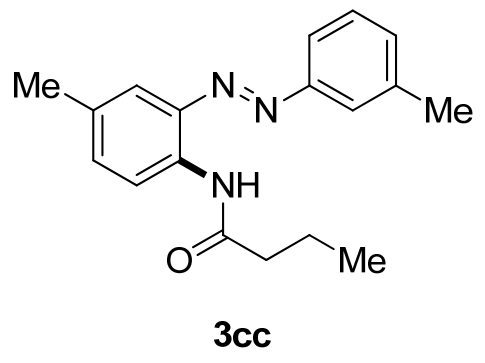

(E)-3-methyl-N-(4-methyl-2-(m-tolyldiazenyl)phenyl)benzamide (3cd): Yield: $48.1 \mathrm{mg}(70 \%) ; R_{f}=$ 0.5 (EtOAc:Hexane = 1:7); Orange solid; Melting point: $118-120{ }^{\circ} \mathrm{C} ;{ }^{1} \mathrm{H} \mathrm{NMR}\left(400 \mathrm{MHz}, \mathrm{CDCl}_{3}\right) \delta$ $11.41(\mathrm{br}, 1 \mathrm{H}), 8.79$ (d, $J=8.5 \mathrm{~Hz}, 1 \mathrm{H}), 7.81-7.80(\mathrm{~m}, 2 \mathrm{H}), 7.77(\mathrm{~d}, J=1.5 \mathrm{~Hz}, 1 \mathrm{H}), 7.72(\mathrm{~s}, 1 \mathrm{H}), 7.71(\mathrm{~d}$, $J=6.1 \mathrm{~Hz}, 1 \mathrm{H}), 7.45-7.31(\mathrm{~m}, 5 \mathrm{H}), 2.48(\mathrm{~s}, 3 \mathrm{H}), 2.47(\mathrm{~s}, 3 \mathrm{H}), 2.43(\mathrm{~s}, 3 \mathrm{H}) ;{ }^{13} \mathrm{C} \mathrm{NMR}\left(100 \mathrm{MHz}, \mathrm{CDCl}_{3}\right)$ $\delta 165.8,152.4,139.2,138.8,138.6,135.4,133.7,133.2,132.8,132.7,132.1,129.1,128.7,127.8,124.5$, 124.4, 122.7, 120.1, 120.0, 21.5, 21.4, 20.8; IR (film): 3395, 3028, 2920, 1674, 1591, 1310, $825 \mathrm{~cm}^{-1}$; HRMS (EI): $m / z$ calcd for $\mathrm{C}_{22} \mathrm{H}_{21} \mathrm{~N}_{3} \mathrm{O}: 343.1685$; found: 343.1686 . 
<smiles>Cc1cccc(/N=N/c2cc(C)ccc2NC(=O)c2cccc(C)c2)c1</smiles>

3cd

(E)-4-methyl-N-(4-methyl-2-(m-tolyldiazenyl)phenyl)benzamide (3ce): Yield: $39.8 \mathrm{mg}(58 \%)$; $\mathrm{R}_{f}=$ 0.5 (EtOAc:Hexane = 1:7); Orange solid; Melting point: $105-107{ }^{\circ} \mathrm{C} ;{ }^{1} \mathrm{H} \mathrm{NMR}\left(400 \mathrm{MHz}, \mathrm{CDCl}_{3}\right) \delta$ 11.32 (br, 1H), 8.78 (d, $J=8.5 \mathrm{~Hz}, 1 \mathrm{H}), 7.89$ (d, $J=8.2 \mathrm{~Hz}, 2 \mathrm{H}), 7.76$ (d, $J=1.3 \mathrm{~Hz}, 1 \mathrm{H}), 7.71(\mathrm{~s}, 1 \mathrm{H})$, $7.70(\mathrm{~d}, J=8.9 \mathrm{~Hz}, 1 \mathrm{H}), 7.43(\mathrm{t}, J=7.6 \mathrm{~Hz}, 1 \mathrm{H}), 7.35-7.32(\mathrm{~m}, 4 \mathrm{H}), 2.48(\mathrm{~s}, 3 \mathrm{H}), 2.45(\mathrm{~s}, 3 \mathrm{H}), 2.42(\mathrm{~s}$, $3 \mathrm{H}) ;{ }^{13} \mathrm{C} \mathrm{NMR}\left(100 \mathrm{MHz}, \mathrm{CDCl}_{3}\right) \delta 165.6,152.5,142.5,139.3,138.8,133.8,133.1,133.1,132.6,132.1$, 129.5, 129.2, 127.3, 124.2, 122.7, 120.1, 120.0, 21.6, 21.5, 20.8; IR (film): 3395, 3027, 2917, 1670, 1611, 1590, 1258, $741 \mathrm{~cm}^{-1}$; HRMS (EI): $m / z$ calcd for $\mathrm{C}_{22} \mathrm{H}_{21} \mathrm{~N}_{3} \mathrm{O}: 343.1685$; found: 343.1685 .<smiles>Cc1ccc(C(=O)Nc2ccc(C)cc2/N=N/c2cccc(C)c2)cc1</smiles>

(E)-2-chloro- $N$-(4-methyl-2-(m-tolyldiazenyl)phenyl)benzamide (3cf): Yield: $30.6 \mathrm{mg}(42 \%)$; $\mathrm{R}_{f}=0.4$ (EtOAc:Hexane = 1:7); Orange solid; Melting point: 100-102 ${ }^{\circ} \mathrm{C} ;{ }^{1} \mathrm{H}$ NMR $\left(400 \mathrm{MHz}, \mathrm{CDCl}_{3}\right) \delta 10.72$ (br, 1H), $8.77(\mathrm{~d}, J=8.5 \mathrm{~Hz}, 1 \mathrm{H}), 7.83(\mathrm{dd}, J=7.2,2.1 \mathrm{~Hz}, 1 \mathrm{H}), 7.70$ (d, $J=1.1 \mathrm{~Hz}, 1 \mathrm{H}), 7.66$ (s, 1H), $7.63(\mathrm{~d}, J=8.4 \mathrm{~Hz}, 1 \mathrm{H}), 7.51-7.49(\mathrm{~m}, 1 \mathrm{H}), 7.47-7.35(\mathrm{~m}, 4 \mathrm{H}), 7.29(\mathrm{~d}, J=7.5 \mathrm{~Hz}, 1 \mathrm{H}), 2.44$ (s, 3H), 2.42 $(\mathrm{s}, 3 \mathrm{H}) ;{ }^{13} \mathrm{C} \mathrm{NMR}\left(100 \mathrm{MHz}, \mathrm{CDCl}_{3}\right) \delta 164.2,152.4,139.3,139.1,135.5,133.81,133.76,133.6,132.1$, 131.8, 130.8, 130.7, 130.6, 129.0, 127.3, 123.1, 120.3 (2C), 120.0, 21.3, 20.9; IR (film): 3367, 3064, 1674, 1592, 1514, 1311, $745 \mathrm{~cm}^{-1}$; HRMS (EI): $\mathrm{m} / z$ calcd for $\mathrm{C}_{21} \mathrm{H}_{18} \mathrm{ClN}_{3} \mathrm{O}: 363.1138$; found: 363.1139 .<smiles>Cc1cccc(/N=N/c2ccc(C)cc2NC(=O)c2ccccc2Cl)c1</smiles> 
(E)-3-chloro- $N$-(4-methyl-2-(m-tolyldiazenyl)phenyl)benzamide (3cg): Yield: $65.5 \mathrm{mg}(90 \%) ; R_{f}=0.6$ (EtOAc: Hexane = 1:7); Orange solid; Melting point: $117-119{ }^{\circ} \mathrm{C} ;{ }^{1} \mathrm{H} \mathrm{NMR}\left(400 \mathrm{MHz}, \mathrm{CDCl}_{3}\right) \delta 11.59$ (br, 1H), $8.74(\mathrm{~d}, J=8.5 \mathrm{~Hz}, 1 \mathrm{H}), 7.95$ (br, 1H), 7.89 (d, $J=7.7 \mathrm{~Hz}, 1 \mathrm{H}), 7.78$ (br, 1H), 7.72 (br, 1H), $7.71(\mathrm{~d}, J=5.9 \mathrm{~Hz}, 1 \mathrm{H}), 7.57-7.54(\mathrm{~m}, 1 \mathrm{H}), 7.47(\mathrm{t}, J=7.9 \mathrm{~Hz}, 1 \mathrm{H}), 7.43(\mathrm{t}, J=8.0 \mathrm{~Hz}, 1 \mathrm{H}), 7.34-7.31$ $(\mathrm{m}, 2 \mathrm{H}), 2.47$ (s, 3H), $2.42(\mathrm{~s}, 3 \mathrm{H}) ;{ }^{13} \mathrm{C} \mathrm{NMR}\left(100 \mathrm{MHz}, \mathrm{CDCl}_{3}\right) \delta 164.2,152.2,139.4,138.7,137.2$, $134.9,133.68,133.65,132.3,132.0,131.9,130.2$, 129.2, 127.2, 126.0, 125.8, 122.8, 120.1, 119.9, 21.4, 20.8; IR (film): 3391, 3067, 2921, 1680, 1589, 1573, 1312, 736, $685 \mathrm{~cm}^{-1}$; HRMS (EI): $\mathrm{m} / z$ calcd for $\mathrm{C}_{21} \mathrm{H}_{18} \mathrm{ClN}_{3} \mathrm{O}$ : 363.1138; found: 363.1136.

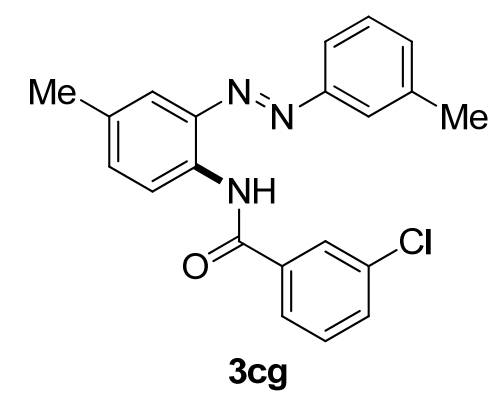

(E)-4-chloro- $N$-(4-methyl-2-(m-tolyldiazenyl)phenyl)benzamide (3ch): Yield: $70.6 \mathrm{mg}(97 \%)$; $\mathrm{R}_{f}=0.6$ (EtOAc:Hexane = 1:7); Orange solid; Melting point: $127-129{ }^{\circ} \mathrm{C} ;{ }^{1} \mathrm{H}$ NMR $\left(400 \mathrm{MHz}, \mathrm{CDCl}_{3}\right) \delta 11.41$ (br, 1H), 8.74 (d, $J=8.5 \mathrm{~Hz}, 1 \mathrm{H}), 7.92$ (d, $J=8.6 \mathrm{~Hz}, 2 \mathrm{H}), 7.77$ (d, $J=1.2 \mathrm{~Hz}, 1 \mathrm{H}), 7.68$ (s, 1H), 7.66 (d, $J=9.9 \mathrm{~Hz}, 1 \mathrm{H}), 7.50$ (d, $J=8.6 \mathrm{~Hz}, 2 \mathrm{H}), 7.44$ (t, $J=7.7 \mathrm{~Hz}, 1 \mathrm{H}), 7.36-7.33(\mathrm{~m}, 2 \mathrm{H}), 2.48(\mathrm{~s}, 3 \mathrm{H}), 2.43(\mathrm{~s}$, $3 \mathrm{H}) ;{ }^{13} \mathrm{C} \mathrm{NMR}\left(100 \mathrm{MHz}, \mathrm{CDCl}_{3}\right) \delta 164.6,152.4,139.4,138.8,138.2,133.8,133.7,133.6,132.5,132.2$, 129.3, 129.1, 128.7, 124.9, 122.6, 120.1, 120.0, 21.5, 20.8; IR (film): 3393, 3029, 2919, 1678, 1595, 1095 , 823, $746 \mathrm{~cm}^{-1}$; HRMS (EI): $m / z$ calcd. for $\mathrm{C}_{21} \mathrm{H}_{18} \mathrm{ClN}_{3} \mathrm{O}$ : 363.1138 ; found: 363.1135 .<smiles>Cc1cccc(/N=N/c2cc(C)ccc2NC(=O)c2ccc(Cl)cc2)c1</smiles>

(E)-3-bromo- $N$-(4-methyl-2-(m-tolyldiazenyl)phenyl)benzamide (3ci): Yield: $75.1 \mathrm{mg}(92 \%) ; \mathrm{R}_{f}=0.6$ (EtOAc:Hexane = 1:7); Orange solid; Melting point: $108-110{ }^{\circ} \mathrm{C} ;{ }^{1} \mathrm{H}$ NMR $\left(400 \mathrm{MHz}, \mathrm{CDCl}_{3}\right) \delta 11.54$ (br, 1H), 8.72 (d, $J=8.5 \mathrm{~Hz}, 1 \mathrm{H}), 8.07$ (t, $J=1.8 \mathrm{~Hz}, 1 \mathrm{H}), 7.92$ (dq, $J=7.8,0.7 \mathrm{~Hz}, 1 \mathrm{H}), 7.75$ (d, $J=1.4$ $\mathrm{Hz}, 1 \mathrm{H}), 7.70-7.68$ (m, 3H), 7.40 (q, J=7.8 Hz, 2H), 7.31 (br, 1H), 7.29 (d, J=1.2 Hz, 1H), 2.47 (s, 3H), $2.40(\mathrm{~s}, 3 \mathrm{H}) ;{ }^{13} \mathrm{C} \mathrm{NMR}\left(100 \mathrm{MHz}, \mathrm{CDCl}_{3}\right) \delta 164.1,152.2,139.4,138.7,137.4,134.9,133.7,133.6,132.3$, 132.0, 130.5, 130.0, 129.2, 126.3, 126.0, 122.9, 122.8, 120.1, 119.9, 21.5, 20.8; IR (film): 3389, 3063, 
2920, 1678, 1596, 1247, 737, $577 \mathrm{~cm}^{-1}$; HRMS (EI): $\mathrm{m} / z$ cald for $\mathrm{C}_{21} \mathrm{H}_{18} \mathrm{BrN}_{3} \mathrm{O}$ : 407.0633; found: 407.0631 .<smiles>Cc1cccc(/N=N/c2cc(C)ccc2NC(=O)c2cccc(Br)c2)c1</smiles>

(E)-4-bromo- $N$-(4-methyl-2-(m-tolyldiazenyl)phenyl)benzamide (3cj): Yield: $71.9 \mathrm{mg}(88 \%)$; $\mathrm{R}_{f}=0.6$ (EtOAc:Hexane = 1:7); Orange solid; Melting point: $142-144{ }^{\circ} \mathrm{C} ;{ }^{1} \mathrm{H}$ NMR $\left(400 \mathrm{MHz}, \mathrm{CDCl}_{3}\right) \delta 11.38$ (br, 1H), $8.72(\mathrm{~d}, J=8.5 \mathrm{~Hz}, 1 \mathrm{H}), 7.83(\mathrm{~d}, J=8.5 \mathrm{~Hz}, 2 \mathrm{H}), 7.75(\mathrm{br}, 1 \mathrm{H}), 7.66-7.63(\mathrm{~m}, 4 \mathrm{H}), 7.42(\mathrm{t}, J=$ $7.6 \mathrm{~Hz}, 1 \mathrm{H}), 7.32(\mathrm{~d}, J=8.0 \mathrm{~Hz}, 2 \mathrm{H}), 2.47(\mathrm{~s}, 3 \mathrm{H}), 2.41(\mathrm{~s}, 3 \mathrm{H}) ;{ }^{13} \mathrm{C} \mathrm{NMR}\left(100 \mathrm{MHz}, \mathrm{CDCl}_{3}\right) \delta 164.6$, $152.3,139.3,138.8,134.2,133.7,133.6,132.4,132.2,132.0,129.3,128.8,126.7,124.9,122.6,120.1$, 119.9, 21.5, 20.8; IR (film): 3330, 2917, 1653, 1594, 1521, 1315, 745, $588 \mathrm{~cm}^{-1}$; HRMS (EI): m/z calcd for $\mathrm{C}_{21} \mathrm{H}_{18} \mathrm{BrN}_{3} \mathrm{O}$ : 407.0633; found: 407.0631 .<smiles>Cc1cccc(/N=N/c2cc(C)ccc2NC(=O)c2ccc(Br)cc2)c1</smiles>

(E)-4-fluoro- $N$-(4-methyl-2-(m-tolyldiazenyl)phenyl)benzamide (3ck): Yield: $68.1 \mathrm{mg}(98 \%) ; R_{f}=0.6$ (EtOAc:Hexane = 1:7); Orange solid; Melting point: $123-125{ }^{\circ} \mathrm{C} ;{ }^{1} \mathrm{HNMR}\left(400 \mathrm{MHz}, \mathrm{CDCl}_{3}\right) \delta 11.37$ (br, $1 \mathrm{H}), 8.73(\mathrm{~d}, J=8.5 \mathrm{~Hz}, 1 \mathrm{H}), 8.01-7.96(\mathrm{~m}, 2 \mathrm{H}), 7.75(\mathrm{~d}, J=1.4 \mathrm{~Hz}, 1 \mathrm{H}), 7.66(\mathrm{br}, 1 \mathrm{H}), 7.65(\mathrm{~d}, J=8.9$ $\mathrm{Hz}, 1 \mathrm{H}), 7.42(\mathrm{t}, J=7.6 \mathrm{~Hz}, 1 \mathrm{H}), 7.34-7.31(\mathrm{~m}, 2 \mathrm{H}), 7.23-7.17(\mathrm{~m}, 2 \mathrm{H}), 2.46(\mathrm{~s}, 3 \mathrm{H}), 2.41(\mathrm{~s}, 3 \mathrm{H}) ;{ }^{13} \mathrm{C}$ NMR $\left(100 \mathrm{MHz}, \mathrm{CDCl}_{3}\right) \delta 165.0\left(\mathrm{~d}, J_{\mathrm{CF}}=252.9 \mathrm{~Hz}\right), 164.6,152.4,139.3,138.8,133.7,133.4,132.6$, $132.2,131.6\left(\mathrm{~d}, J_{\mathrm{CF}}=3.0 \mathrm{~Hz}\right), 129.6\left(\mathrm{~d}, J_{\mathrm{CF}}=8.9 \mathrm{~Hz}\right), 129.2,124.9,122.6,120.1,119.9,115.9\left(\mathrm{~d}, J_{\mathrm{CF}}=\right.$ 21.9 Hz), 21.5, 20.8; ${ }^{19} \mathrm{~F}$ NMR (376 MHz, $\mathrm{CDCl}_{3}$ ) $\delta$-107.4; IR (film): 3353, 3074, 1677, 1597, 1315, 1225, $791 \mathrm{~cm}^{-1}$; HRMS (EI): $m / z$ calcd for $\mathrm{C}_{21} \mathrm{H}_{18} \mathrm{FN}_{3} \mathrm{O}$ : 347.1434; found: 347.1436 . 


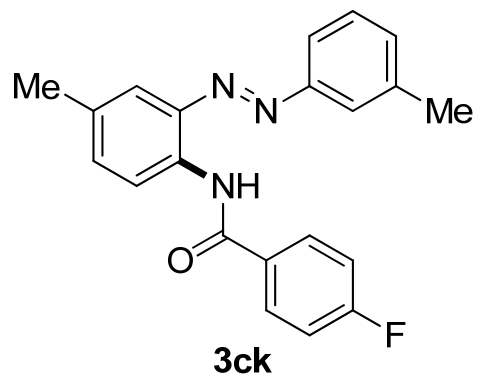

(E)- $N$-(4-methyl-2-(m-tolyldiazenyl)phenyl)-4-nitrobenzamide (3cl): Yield: $65.9 \mathrm{mg}(88 \%) ; R_{f}=0.5$ (EtOAc:Hexane = 1:7); Orange solid; Melting point: $163-165{ }^{\circ} \mathrm{C} ;{ }^{1} \mathrm{HNMR}\left(400 \mathrm{MHz}, \mathrm{CDCl}_{3}\right) \delta 11.60$ (br, $1 \mathrm{H}), 8.72(\mathrm{~d}, J=8.4 \mathrm{~Hz}, 1 \mathrm{H}), 8.38(\mathrm{~d}, J=8.6 \mathrm{~Hz}, 2 \mathrm{H}), 8.13(\mathrm{~d}, J=8.6 \mathrm{~Hz}, 2 \mathrm{H}), 7.79(\mathrm{~s}, 1 \mathrm{H}), 7.66(\mathrm{~s}, 1 \mathrm{H})$, $7.63(\mathrm{~d}, J=8.0 \mathrm{~Hz}, 1 \mathrm{H}), 7.45(\mathrm{t}, J=7.6 \mathrm{~Hz}, 1 \mathrm{H}), 7.35(\mathrm{~d}, J=6.9 \mathrm{~Hz}, 2 \mathrm{H}), 2.48(\mathrm{~s}, 3 \mathrm{H}), 2.44(\mathrm{~s}, 3 \mathrm{H}) ;{ }^{13} \mathrm{C}$ NMR $\left(100 \mathrm{MHz}, \mathrm{CDCl}_{3}\right) \delta 163.4,152.2,149.8,140.9,139.5,138.8,134.2,133.7,132.4,131.8,129.3$, 128.4, 125.6, 124.0, 122.6, 120.1, 119.8, 21.5, 20.8; IR (film): 3347, 2918, 1657, 1594, 1521, 1346, 1313, $710 \mathrm{~cm}^{-1}$; HRMS (EI): $\mathrm{m} / z$ calcd for $\mathrm{C}_{21} \mathrm{H}_{18} \mathrm{~N}_{4} \mathrm{O}_{3}: 374.1379$; found: 374.1381 .

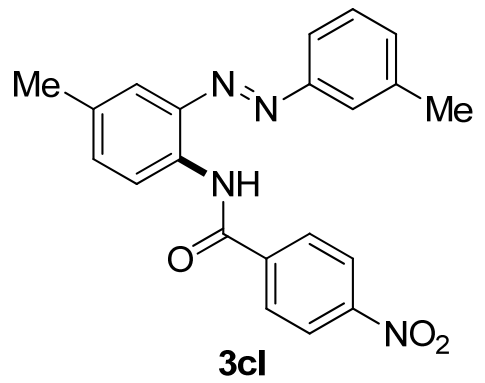

(E)- $N$-(4-methyl-2-(m-tolyldiazenyl)phenyl)furan-2-carboxamide (3cm): Yiel : $31.9 \mathrm{mg}(50 \%) ; R_{f}=$ 0.2 (EtOAc:Hexane = 1:7); Yellow solid; Melting point: $128-129{ }^{\circ} \mathrm{C} ;{ }^{1} \mathrm{H}$ NMR $\left(400 \mathrm{MHz}, \mathrm{CDCl}_{3}\right) \delta$ 11.33 (br, 1H), 8.70 (d, $J=8.4 \mathrm{~Hz}, 1 \mathrm{H}), 7.81(\mathrm{~s}, 1 \mathrm{H}), 7.80(\mathrm{~d}, J=9.0 \mathrm{~Hz}, 1 \mathrm{H}), 7.74(\mathrm{~d}, J=1.3 \mathrm{~Hz}, 1 \mathrm{H})$, $7.58(\mathrm{dd}, J=1.7,0.8 \mathrm{~Hz}, 1 \mathrm{H}), 7.45(\mathrm{t}, J=7.6 \mathrm{~Hz}, 1 \mathrm{H}), 7.34-7.32(\mathrm{~m}, 2 \mathrm{H}), 7.27(\mathrm{dd}, J=3.5,0.7 \mathrm{~Hz}, 1 \mathrm{H})$, $6.60(\mathrm{q}, J=1.7 \mathrm{~Hz}, 1 \mathrm{H}), 2.50(\mathrm{~s}, 3 \mathrm{H}), 2.41(\mathrm{~s}, 3 \mathrm{H}) ;{ }^{13} \mathrm{C} \mathrm{NMR}\left(100 \mathrm{MHz}, \mathrm{CDCl}_{3}\right) \delta 156.2,152.5,148.5$, $144.2,139.1,138.9,133.6,133.4,132.9,132.1,129.1,123.0,122.5,120.3,120.2,115.2,112.7,21.5$, 20.9; IR (film): 3363, 2920, 1676, 1597, 1520, 1312, $1163 \mathrm{~cm}^{-1}$; HRMS (EI): $\mathrm{m} / z$ calcd for $\mathrm{C}_{19} \mathrm{H}_{17} \mathrm{~N}_{3} \mathrm{O}_{2}$ : 319.1321; found: 319.1321 .<smiles>Cc1cccc(N=Nc2cc(C)ccc2NC(=O)c2ccco2)c1</smiles> 
(E)- $N$-(4-methyl-2-(m-tolyldiazenyl)phenyl)thiophene-2-carboxamide (3cn): Yield: $61.0 \mathrm{mg}(91 \%) ; R_{f}$ $=0.4\left(\right.$ EtOAc:Hexane = 1:7); Orange solid; Melting point: $106-108{ }^{\circ} \mathrm{C} ;{ }^{1} \mathrm{H} \mathrm{NMR}\left(400 \mathrm{MHz}, \mathrm{CDCl}_{3}\right) \delta$ $11.08(\mathrm{br}, 1 \mathrm{H}), 8.67(\mathrm{~d}, J=8.5 \mathrm{~Hz}, 1 \mathrm{H}), 7.72-7.70(\mathrm{~m}, 4 \mathrm{H}), 7.57(\mathrm{~d}, J=4.9 \mathrm{~Hz}, 1 \mathrm{H}), 7.43$ (t, $J=7.8 \mathrm{~Hz}$, 1H), $7.32(\mathrm{~s}, 1 \mathrm{H}), 7.30(\mathrm{~s}, 1 \mathrm{H}), 7.16(\mathrm{t}, J=4.3 \mathrm{~Hz}, 1 \mathrm{H}), 2.47(\mathrm{~s}, 3 \mathrm{H}), 2.40(\mathrm{~s}, 3 \mathrm{H}) ;{ }^{13} \mathrm{C} \mathrm{NMR}(100 \mathrm{MHz}$, $\left.\mathrm{CDCl}_{3}\right) \delta 159.9,152.5,140.0,139.3,138.6,133.7,133.3,132.8,132.1,130.8,129.2,128.7,127.9,123.6$, 122.8, 120.1, 119.9, 21.5, 20.8; IR (film): 3383, 3100, 2919, 1665, 1592, 1512, 1311, $1248 \mathrm{~cm}^{-1}$; HRMS (EI): $m / z$ calcd for $\mathrm{C}_{19} \mathrm{H}_{17} \mathrm{~N}_{3} \mathrm{OS}$ : 335.1092; found: 335.1095 .

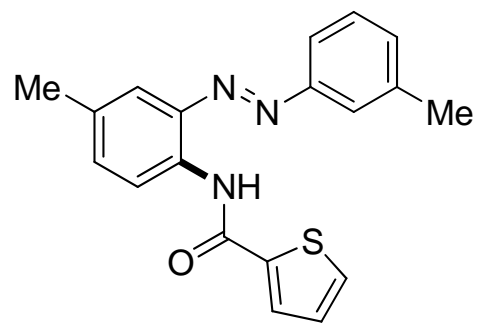

$3 \mathrm{cn}$

\section{General procedure for diamidation of azobenzene with dioxazolones}<smiles>c1ccc(/N=N/c2ccccc2)cc1</smiles>

$1 a$

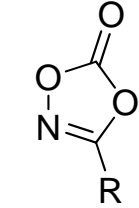

2
$\left[\mathrm{Cp}^{\star} \mathrm{RhCl}_{2}\right]_{2}(2.5 \mathrm{~mol} \%)$ $\operatorname{AgSbF}_{6}(10.0 \mathrm{~mol} \%)$ $\underset{\text { DiOAC }(10.0 \mathrm{~mol} \%)}{\stackrel{2}{\circ}{ }^{\circ} \mathrm{C}, 12 \mathrm{~h}}$ - $\mathrm{CO}_{2}$ under air<smiles>[R]C(=O)Nc1cccc(NC([R])=O)c1N=Nc1ccccc1</smiles>

To a test tube were added azobenzene (1a) $(36.5 \mathrm{mg}, 0.2 \mathrm{mmol})$, dioxazolone (2) (97.9 mg, $0.6 \mathrm{mmol})$, $\left[\mathrm{Cp}^{*} \mathrm{RhCl}_{2}\right]_{2}(3.1 \mathrm{mg}, 0.005 \mathrm{mmol}), \mathrm{AgSbF}_{6}(6.9 \mathrm{mg}, 0.02 \mathrm{mmol})$, and LiOAc $(1.3 \mathrm{mg}, 0.02 \mathrm{mmol}) \mathrm{in}$ DCE $(1.0 \mathrm{~mL})$. The resulting mixture was stirred at $25{ }^{\circ} \mathrm{C}$ for $12 \mathrm{~h}$ under air. After celite filtration and evaporation of the solvent in vacuo, the crude product was purified by column chromatography on silica gel using EtOAc:Hexane = 1:7.

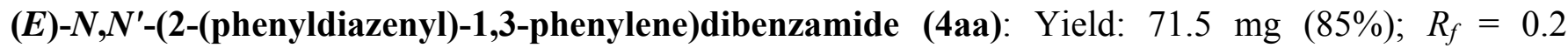
(EtOAc:Hexane = 1:7); Brown solid; Melting point: 227-229 ${ }^{\circ} \mathrm{C} ;{ }^{1} \mathrm{HNMR}\left(400 \mathrm{MHz}, \mathrm{CDCl}_{3}\right) \delta 11.76$ (br, 2H), $8.66(\mathrm{~d}, J=8.4 \mathrm{~Hz}, 2 \mathrm{H}), 8.00(\mathrm{~d}, J=7.0 \mathrm{~Hz}, 4 \mathrm{H}), 7.82(\mathrm{~d}, J=1.7 \mathrm{~Hz}, 1 \mathrm{H}), 7.80(\mathrm{~d}, J=1.3 \mathrm{~Hz}, 1 \mathrm{H})$, 7.64-7.54 (m, 10H); ${ }^{13} \mathrm{C}$ NMR $\left(100 \mathrm{MHz}, \mathrm{CDCl}_{3}\right) \delta 165.9,152.2,136.2,136.0,135.3,132.2,131.6,129.8$, 128.9, 127.3, 126.2, 121.8, 114.6; IR (film): 3362, 3063, 1684, 1594, 1579, 1302, $776 \mathrm{~cm}^{-1}$; HRMS (EI): $m / z$ calcd for $\mathrm{C}_{26} \mathrm{H}_{20} \mathrm{~N}_{4} \mathrm{O}_{2}$ : 420.1586; found: 420.1582 . 
<smiles>CC(C)(C)C(=O)Nc1cccc(NC(=O)c2ccccc2)c1N=Nc1ccccc1</smiles>

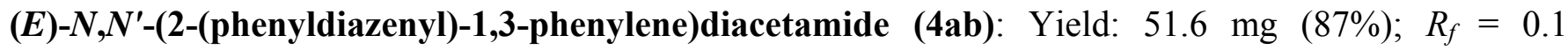
(EtOAc:Hexane = 1:7); Yellow solid; Melting point: 231-233 ${ }^{\circ} \mathrm{C} ;{ }^{1} \mathrm{HNMR}\left(400 \mathrm{MHz}, \mathrm{CDCl}_{3}\right) \delta 10.83$ (br, 2H), $8.38(\mathrm{~d}, J=8.4 \mathrm{~Hz}, 2 \mathrm{H}), 7.76-7.73(\mathrm{~m}, 2 \mathrm{H}), 7.61-7.52(\mathrm{~m}, 3 \mathrm{H}), 7.45(\mathrm{t}, J=8.4 \mathrm{~Hz}, 1 \mathrm{H}), 2.26(\mathrm{~s}, 6 \mathrm{H})$; ${ }^{13} \mathrm{C} \mathrm{NMR}\left(100 \mathrm{MHz}, \mathrm{CDCl}_{3}\right) \delta 168.8,151.8,135.7,135.5,131.6,129.7,129.2,125.2,122.0,114.4,25.6$; IR (film): 3317, 3060, 1673, 1592, 1460, 1296, $770 \mathrm{~cm}^{-1}$; HRMS (EI): $\mathrm{m} / z$ calcd for $\mathrm{C}_{16} \mathrm{H}_{16} \mathrm{~N}_{4} \mathrm{O}_{2}$ : 296.1273; found: 296.1271 .<smiles>CC(=O)Nc1cccc(NC(C)=O)c1/N=N/c1ccccc1</smiles>

$4 a b$

\section{General procedure for amidation of mono-amidated azobenzenes}<smiles>O=C(Nc1ccccc1N=Nc1ccccc1)c1ccccc1</smiles>

3aa<smiles>[R]c1noc(=O)o1</smiles>

2
$\left[\mathrm{Cp}^{*} \mathrm{RhCl}_{2}\right]_{2}(2.5 \mathrm{~mol} \%)$ $\mathrm{AgSbF}_{6}(10.0 \mathrm{~mol} \%)$ $\underset{\operatorname{LiOAC}(10.0 \mathrm{~mol} \%)}{\stackrel{\mathrm{DCE}, 25^{\circ} \mathrm{C}, 1 \mathrm{~h}}{\longrightarrow}}$ $-\mathrm{CO}_{2}$ under air<smiles>[R]C(=O)Nc1cccc(NC(=O)c2ccccc2)c1N=Nc1ccccc1</smiles>

To a test tube were added mono-amidated azobenzene (3aa) (120.5 mg, $0.4 \mathrm{mmol}$ ), dioxazolone (2) (20.2 mg, $0.2 \mathrm{mmol}),\left[\mathrm{Cp}^{*} \mathrm{RhCl}_{2}\right]_{2}$ (3.1 mg, $\left.0.005 \mathrm{mmol}\right), \mathrm{AgSbF}_{6}(6.9 \mathrm{mg}, 0.02 \mathrm{mmol})$, and LiOAc (1.3 $\mathrm{mg}, 0.02 \mathrm{mmol})$ in DCE $(1.0 \mathrm{~mL})$. The resulting mixture was stirred at $25{ }^{\circ} \mathrm{C}$ for $1 \mathrm{~h}$ under air. After celite filtration and evaporation of the solvent in vacuo, the crude product was purified by column chromatography on silica gel using EtOAc:Hexane = 1:2. 
(E)- $N$-(3-acetamido-2-(phenyldiazenyl)phenyl)benzamide (5ab): Yield: $43.8 \mathrm{mg}(61 \%) ; R_{f}=0.3$ (EtOAc:Hexane = 1:2); Brown solid; Melting point: 192-194 ${ }^{\circ} \mathrm{C} ;{ }^{1} \mathrm{HNMR}\left(400 \mathrm{MHz}, \mathrm{CDCl}_{3}\right) \delta 12.06$ (br, 1H), 10.45 (br, 1H), 8.60 (d, $J=8.4 \mathrm{~Hz}, 1 \mathrm{H}), 8.42$ (d, $J=8.4 \mathrm{~Hz}, 1 \mathrm{H}), 7.97$ (d, $J=7.9 \mathrm{~Hz}, 2 \mathrm{H}), 7.76(\mathrm{~d}, J$ $=8.1 \mathrm{~Hz}, 2 \mathrm{H}), 7.62-7.49(\mathrm{~m}, 7 \mathrm{H}), 2.28(\mathrm{~s}, 3 \mathrm{H}) ;{ }^{13} \mathrm{C} \mathrm{NMR}\left(100 \mathrm{MHz}, \mathrm{CDCl}_{3}\right) \delta 168.5,166.2,152.0,136.9$, 135.7, 135.4, 135.0, 132.1, 131.6, 129.7, 129.2, 128.9, 127.3, 125.8, 121.9, 114.5, 25.5; IR (film): 3303, 3062, 1681, 1590, 1459, 1285, $770 \mathrm{~cm}^{-1}$; HRMS (EI): $\mathrm{m} / z$ calcd for $\mathrm{C}_{21} \mathrm{H}_{18} \mathrm{~N}_{4} \mathrm{O}_{2}$ : 358.1430; found: 358.1433 .

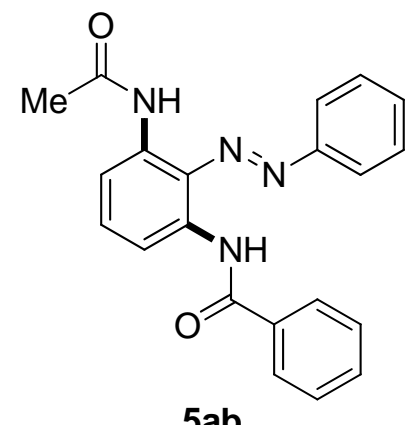

(E)-N-(3-benzamido-2-(phenyldiazenyl)phenyl)-3-chlorobenzamide (5ag): Yield: $57.1 \mathrm{mg}(63 \%) ; R_{f}=$ 0.3 (EtOAc:Hexane = 1:2); Yellow solid; Melting point: 209-211 ${ }^{\circ} \mathrm{C} ;{ }^{1} \mathrm{H} \mathrm{NMR}\left(400 \mathrm{MHz}, \mathrm{CDCl}_{3}\right) \delta$ 11.81 (br, 1H), 11.63 (br, 1H), 8.66 (dd, $J=8.4,1.0 \mathrm{~Hz}, 1 \mathrm{H}), 8.61$ (dd, $J=8.4,1.0 \mathrm{~Hz}, 1 \mathrm{H}), 8.00-7.96$ (m, 3H), $7.90(\mathrm{~d}, J=7.8 \mathrm{~Hz}, 1 \mathrm{H}), 7.83(\mathrm{dd}, J=8.1,1.4 \mathrm{~Hz}, 2 \mathrm{H}), 7.63-7.53(\mathrm{~m}, 8 \mathrm{H}), 7.49$ (t, $J=7.8 \mathrm{~Hz}, 1 \mathrm{H})$; ${ }^{13} \mathrm{C} \mathrm{NMR}\left(100 \mathrm{MHz}, \mathrm{CDCl}_{3}\right) \delta 165.9,164.3,152.1,137.0,136.5,135.9,135.7,135.3,135.0,132.23$, 132.17, 131.8, 130.3, 129.8, 128.9, 127.3, 127.2, 126.2, 125.8, 121.9, 114.9, 114.5; IR (film): 3371, 3060, 1684, 1612, 1592, 1298, 768, $680 \mathrm{~cm}^{-1}$; HRMS (EI): $\mathrm{m} / z$ calcd for $\mathrm{C}_{26} \mathrm{H}_{19} \mathrm{ClN}_{4} \mathrm{O}_{2}$ : 454.1197; found: 454.1201.<smiles>O=C(Nc1cccc(NC(=O)c2ccccc2)c1/N=N/c1ccccc1)c1ccccc1</smiles>

5 ag

(E)-N-(3-benzamido-2-(phenyldiazenyl)phenyl)-4-bromobenzamide (5aj): Yield: $75.8 \mathrm{mg}(76 \%) ; R_{f}=$ 0.3 (EtOAc:Hexane = 1:7); Yellow solid; Melting point: $215-217{ }^{\circ} \mathrm{C} ;{ }^{1} \mathrm{H} \mathrm{NMR}\left(400 \mathrm{MHz}, \mathrm{CDCl}_{3}\right) \delta$ 11.76 (br, 1H), 11.64 (br, 1H), $8.66(\mathrm{~d}, J=8.4 \mathrm{~Hz}, 1 \mathrm{H}), 8.61(\mathrm{~d}, J=8.4 \mathrm{~Hz}, 1 \mathrm{H}), 7.85(\mathrm{~d}, J=8.4 \mathrm{~Hz}, 2 \mathrm{H})$, $7.78(\mathrm{~d}, J=7.0 \mathrm{~Hz}, 2 \mathrm{H}), 7.68(\mathrm{~d}, J=8.4 \mathrm{~Hz}, 2 \mathrm{H}), 7.63-7.53(\mathrm{~m}, 7 \mathrm{H}) ;{ }^{13} \mathrm{C} \mathrm{NMR}\left(100 \mathrm{MHz}, \mathrm{CDCl}_{3}\right) \delta$ $165.9,165.0,152.2,136.5,136.0,135.8,135.3,134.2,132.25,132.16,131.7,129.9,128.93,128.86$, 
127.3, 127.0, 126.2, 121.7, 114.8, 114.6; IR (film): 3344, 3060, 1683, 1589, 1478, 1295, 697, $614 \mathrm{~cm}^{-1}$; HRMS (EI): $m / z$ calcd for $\mathrm{C}_{26} \mathrm{H}_{19} \mathrm{BrN}_{4} \mathrm{O}_{2}$ : 498.0691; found: 498.0689.<smiles>O=C(Nc1cccc(NC(=O)c2ccc(Br)cc2)c1N=Nc1ccccc1)c1ccccc1</smiles>

5aj

(E)- $N$-(3-benzamido-2-(phenyldiazenyl)phenyl)thiophene-2-carboxamide (5an): Yield: $47.7 \mathrm{mg}$ $(56 \%) ; R_{f}=0.2$ (EtOAc:Hexane $\left.=1: 2\right)$; Yellow solid; Melting point: $214-216{ }^{\circ} \mathrm{C} ;{ }^{1} \mathrm{H}$ NMR $(400 \mathrm{MHz}$, $\left.\mathrm{CDCl}_{3}\right) \delta 11.84$ (br, 1H), $11.43(\mathrm{br}, 1 \mathrm{H}), 8.63(\mathrm{dd}, J=8.4,1.1 \mathrm{~Hz}, 1 \mathrm{H}), 8.54(\mathrm{dd}, J=8.4,1.1 \mathrm{~Hz}, 1 \mathrm{H})$, $7.98(\mathrm{~d}, J=7.0 \mathrm{~Hz}, 2 \mathrm{H}), 7.85(\mathrm{dd}, J=8.1,1.4 \mathrm{~Hz}, 2 \mathrm{H}), 7.74(\mathrm{dd}, J=3.7,1.1 \mathrm{~Hz}, 1 \mathrm{H}), 7.63-7.53(\mathrm{~m}, 8 \mathrm{H})$, $7.18(\mathrm{dd}, J=4.9,3.8 \mathrm{~Hz}, 1 \mathrm{H}) ;{ }^{13} \mathrm{C} \mathrm{NMR}\left(100 \mathrm{MHz}, \mathrm{CDCl}_{3}\right) \delta 166.0,160.1,152.3,139.7,136.2,135.91$, $135.85,135.3$, 132.2, 131.6, 131.1, 129.8, 129.2, 128.9, 128.0, 127.3, 126.0, 121.8, 114.62, 114.59; IR (film): 3363, 3063, 1682, 1592, 1298, $703 \mathrm{~cm}^{-1}$; HRMS (EI): $\mathrm{m} / z$ calcd for $\mathrm{C}_{24} \mathrm{H}_{18} \mathrm{~N}_{4} \mathrm{O}_{2} \mathrm{~S}: 426.1150$; found: 426.1154 .<smiles>O=C(Nc1cccc(NC(=O)c2cccs2)c1N=Nc1ccccc1)c1ccccc1</smiles>

5an 


\section{Studies with isotopically labeled compounds}
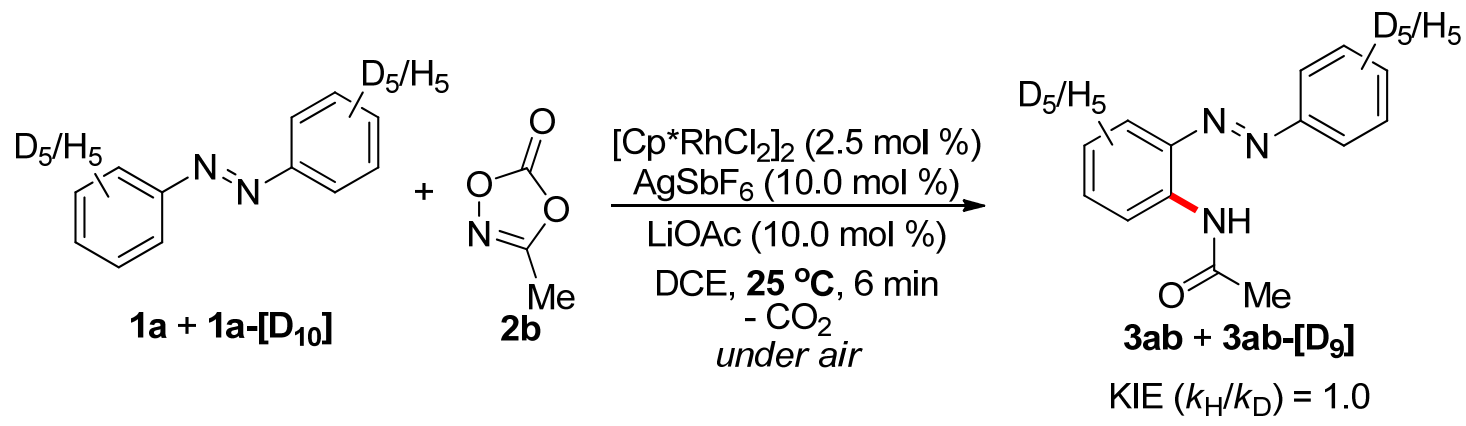

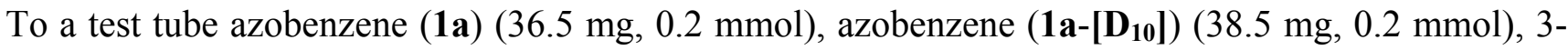
methyl-1,4,2-dioxazol-5-one (2b) (20.2 mg, $0.2 \mathrm{mmol}),\left[\mathrm{Cp} * \mathrm{RhCl}_{2}\right]_{2}$ (3.1 mg, 0.005 mmol), AgSbF 6 (6.9 $\mathrm{mg}, 0.02 \mathrm{mmol})$, and LiOAc $(1.3 \mathrm{mg}, 0.02 \mathrm{mmol})$ in DCE $(1.0 \mathrm{~mL})$ were added. The resulting mixture was stirred under air at $25{ }^{\circ} \mathrm{C}$ for $6 \mathrm{~min}$. After celite filtration and evaporation of the solvent in vacuo, the crude product was purified by column chromatography on silica gel using EtOAc:Hexane = 1:7 to yield

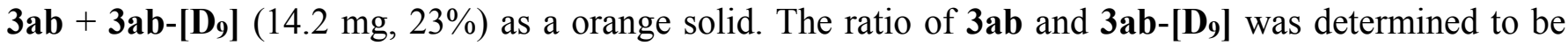
50/50 (1.0) by ${ }^{1} \mathrm{H}$ NMR spectroscopy. 


\section{X-ray crystallography of 3aa}

A pink block-like specimen of $\mathrm{C}_{19} \mathrm{H}_{15} \mathrm{~N}_{3} \mathrm{O}$, approximate dimensions $0.080 \mathrm{~mm} \times 0.200 \mathrm{~mm} \times 0.360 \mathrm{~mm}$, was used for the X-ray crystallographic analysis. The X-ray intensity data were measured. Structural information was deposited at the Cambridge Crystallographic Data Center (CCDC 1477470).

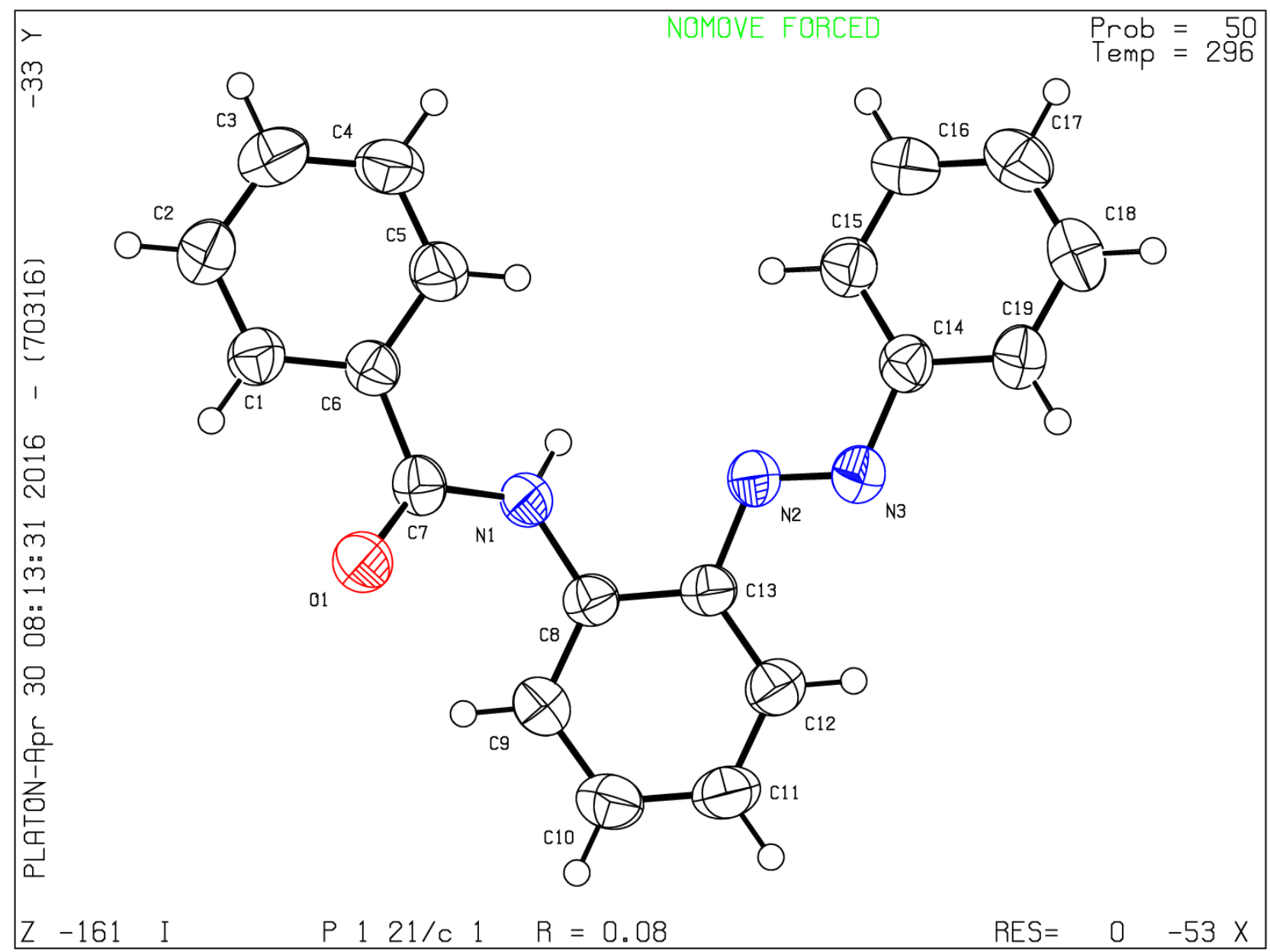

Table 1. Sample and crystal data for 3aa.

\begin{tabular}{|c|c|c|}
\hline Identification code & \multicolumn{2}{|l|}{$3 \mathrm{aa}$} \\
\hline Chemical formula & \multicolumn{2}{|l|}{$\mathrm{C}_{19} \mathrm{H}_{15} \mathrm{~N}_{3} \mathrm{O}$} \\
\hline Formula weight & \multicolumn{2}{|l|}{301.34} \\
\hline Temperature & \multicolumn{2}{|l|}{$296(2) \mathrm{K}$} \\
\hline Wavelength & \multicolumn{2}{|l|}{$0.71073 \AA$} \\
\hline Crystal size & \multicolumn{2}{|c|}{$0.080 \times 0.200 \times 0.360 \mathrm{~mm}$} \\
\hline Crystal habit & \multicolumn{2}{|l|}{ pink block } \\
\hline Crystal system & \multicolumn{2}{|l|}{ monoclinic } \\
\hline Space group & \multicolumn{2}{|l|}{$\mathrm{P} 122_{1} / \mathrm{c} 1$} \\
\hline \multirow[t]{2}{*}{ Unit cell dimensions } & $\mathrm{a}=17.259(2) \AA$ & $\alpha=90^{\circ}$ \\
\hline & $\mathrm{b}=4.9915(6) \AA$ & $\beta=111.712(4)^{\circ}$ \\
\hline
\end{tabular}




\begin{tabular}{|c|c|c|}
\hline & $\mathrm{c}=18.893(2) \AA$ & $\gamma=90^{\circ}$ \\
\hline Volume & $1512.1(3) \AA^{3}$ & \\
\hline $\mathbf{Z}$ & 4 & \\
\hline Density (calculated) & $1.324 \mathrm{~g} / \mathrm{cm}^{3}$ & \\
\hline Absorption coefficient & $0.084 \mathrm{~mm}^{-1}$ & \\
\hline$F(\mathbf{0 0 0 )}$ & 632 & \\
\hline
\end{tabular}

Table 2. Data collection and structure refinement for $3 \mathrm{aa}$.

\begin{tabular}{|c|c|c|}
\hline Theta range for data collection & \multicolumn{2}{|l|}{2.32 to $28.42^{\circ}$} \\
\hline Index ranges & \multicolumn{2}{|c|}{$-23<=\mathrm{h}<=23,-6<=\mathrm{k}<=6,-25<=\mathrm{l}<=24$} \\
\hline Reflections collected & \multicolumn{2}{|l|}{30648} \\
\hline Independent reflections & \multicolumn{2}{|c|}{$3524[\mathrm{R}(\mathrm{int})=0.1233]$} \\
\hline Coverage of independent reflections & \multicolumn{2}{|l|}{$97.4 \%$} \\
\hline Absorption correction & \multicolumn{2}{|l|}{ multi-scan } \\
\hline Max. and min. transmission & \multicolumn{2}{|l|}{0.9930 and 0.9700} \\
\hline Refinement method & \multicolumn{2}{|c|}{ Full-matrix least-squares on $\mathrm{F}^{2}$} \\
\hline Refinement program & \multicolumn{2}{|c|}{ SHELXL-2013 (Sheldrick, 2013) } \\
\hline Function minimized & \multicolumn{2}{|l|}{$\sum \mathrm{w}\left(\mathrm{F}_{\mathrm{o}}^{2}-\mathrm{F}_{\mathrm{c}}^{2}\right)^{2}$} \\
\hline Data / restraints / parameters & \multicolumn{2}{|l|}{$3524 / 0 / 209$} \\
\hline Goodness-of-fit on $\mathbf{F}^{2}$ & \multicolumn{2}{|l|}{1.034} \\
\hline \multirow[t]{2}{*}{ Final $\mathbf{R}$ indices } & 2021 data; $\mathrm{I}>2 \sigma(\mathrm{I})$ & $\mathrm{R} 1=0.0777, \mathrm{wR} 2=0.1899$ \\
\hline & all data & $\mathrm{R} 1=0.1369, \mathrm{wR} 2=0.2149$ \\
\hline Weighting scheme & \multicolumn{2}{|c|}{$\begin{array}{l}\mathrm{W}=1 /\left[\sigma^{2}\left(\mathrm{~F}_{\mathrm{o}}^{2}\right)+(0.1184 \mathrm{P})^{2}+0.0366 \mathrm{P}\right] \\
\text { where } \mathrm{P}=\left(\mathrm{F}_{\mathrm{o}}{ }^{2}+2 \mathrm{~F}_{\mathrm{c}}{ }^{2}\right) / 3\end{array}$} \\
\hline Extinction coefficient & \multicolumn{2}{|l|}{$0.0270(50)$} \\
\hline Largest diff. peak and hole & \multicolumn{2}{|c|}{0.276 and $-0.227 \mathrm{e}^{-3}$} \\
\hline R.M.S. deviation from mean & \multicolumn{2}{|l|}{$0.074 \mathrm{e}^{-3}$} \\
\hline
\end{tabular}

Table 3. Bond lengths (Å) for 3aa.

\begin{tabular}{|c|c|c|c|}
\hline $\mathrm{O} 1-\mathrm{C} 7$ & $1.233(2)$ & N2-N3 & $1.257(3)$ \\
\hline N2-C13 & $1.427(3)$ & N3-C14 & $1.429(3)$ \\
\hline $\mathrm{N} 1-\mathrm{C} 7$ & $1.351(3)$ & N1-C8 & $1.414(3)$ \\
\hline N1-H1 & 0.86 & С8-C9 & $1.389(3)$ \\
\hline C8-C13 & $1.398(3)$ & C14-C19 & $1.379(3)$ \\
\hline C14-C15 & $1.386(3)$ & C13-C12 & $1.395(3)$ \\
\hline C6-C1 & $1.382(3)$ & C6-C5 & $1.389(3)$ \\
\hline C6-C7 & $1.500(3)$ & C9-C10 & $1.388(3)$ \\
\hline C9-H9 & 0.93 & $\mathrm{C} 1-\mathrm{C} 2$ & $1.375(3)$ \\
\hline C1-H1A & 0.93 & C16-C17 & $1.372(4)$ \\
\hline C16-C15 & $1.380(3)$ & C16-H16 & 0.93 \\
\hline C15-H15 & 0.93 & C12-C11 & $1.375(4)$ \\
\hline C12-H12 & 0.93 & C5-C4 & $1.373(4)$ \\
\hline C5-H5 & 0.93 & C19-C18 & $1.387(4)$ \\
\hline C19-H19 & 0.93 & C17-C18 & $1.373(4)$ \\
\hline C17-H17 & 0.93 & C10-C11 & $1.381(4)$ \\
\hline C10-H10 & 0.93 & C18-H18 & 0.93 \\
\hline
\end{tabular}




\begin{tabular}{||l|l|l|l||}
\hline C2-C3 & $1.380(4)$ & C2-H2 & 0.93 \\
\hline \hline C11-H11 & 0.93 & C3-C4 & $1.374(4)$ \\
\hline C3-H3 & 0.93 & C4-H4 & 0.93 \\
\hline
\end{tabular}

Table 4. Bond angles $\left({ }^{\circ}\right)$ for 3 aa.

\begin{tabular}{|l||l|l|l||}
\hline N3-N2-C13 & $114.06(18)$ & N2-N3-C14 & $114.67(19)$ \\
\hline C7-N1-C8 & $126.72(19)$ & C7-N1-H1 & 116.6 \\
\hline C8-N1-H1 & 116.6 & C9-C8-C13 & $119.7(2)$ \\
\hline C9-C8-N1 & $122.1(2)$ & C13-C8-N1 & $118.17(19)$ \\
\hline C19-C14-C15 & $119.6(2)$ & C19-C14-N3 & $115.3(2)$ \\
\hline C15-C14-N3 & $125.0(2)$ & C12-C13-C8 & $119.8(2)$ \\
\hline C12-C13-N2 & $123.3(2)$ & C8-C13-N2 & $116.88(19)$ \\
\hline C1-C6-C5 & $118.9(2)$ & C1-C6-C7 & $117.3(2)$ \\
\hline C5-C6-C7 & $123.7(2)$ & C10-C9-C8 & $119.4(2)$ \\
\hline C10-C9-H9 & 120.3 & C8-C9-H9 & 120.3 \\
\hline O1-C7-N1 & $123.2(2)$ & O1-C7-C6 & $120.5(2)$ \\
\hline N1-C7-C6 & $116.33(19)$ & C2-C1-C6 & $121.2(2)$ \\
\hline C2-C1-H1A & 119.4 & C6-C1-H1A & 119.4 \\
\hline C17-C16-C15 & $120.2(2)$ & C17-C16-H16 & 119.9 \\
\hline C15-C16-H16 & 119.9 & C16-C15-C14 & $119.7(2)$ \\
\hline C16-C15-H15 & 120.2 & C14-C15-H15 & 120.2 \\
\hline C11-C12-C13 & $120.4(2)$ & C11-C12-H12 & 119.8 \\
\hline C13-C12-H12 & 119.8 & C4-C5-C6 & $120.1(2)$ \\
\hline C4-C5-H5 & 120.0 & C6-C5-H5 & 120.0 \\
\hline C14-C19-C18 & $120.6(2)$ & C14-C19-H19 & 119.7 \\
\hline \hline C18-C19-H19 & 119.7 & C16-C17-C18 & $120.8(2)$ \\
\hline C16-C17-H17 & 119.6 & C18-C17-H17 & 119.6 \\
\hline C11-C10-C9 & $121.2(2)$ & C11-C10-H10 & 119.4 \\
\hline C9-C10-H10 & 119.4 & C17-C18-C19 & $119.1(3)$ \\
\hline C17-C18-H18 & 120.5 & C19-C18-H18 & 120.5 \\
\hline C1-C2-C3 & $119.1(2)$ & C1-C2-H2 & 120.4 \\
\hline C3-C2-H2 & 120.4 & C12-C11-C10 & $119.6(2)$ \\
\hline C12-C11-H11 & 120.2 & C10-C11-H11 & 120.2 \\
\hline C4-C3-C2 & $120.4(2)$ & C4-C3-H3 & 119.8 \\
\hline C2-C3-H3 & 119.8 & C5-C4-C3 & $120.3(2)$ \\
\hline C5-C4-H4 & 119.9 & C3-C4-H4 & 119.9 \\
\hline & & \\
\hline
\end{tabular}

\section{References}

(1) (a) Zhang, C.; Jiao, N. Angew. Chem., Int. Ed. 2010, 49, 6174. (b) Ellman, J. A.; Lavis, L. D.; Bergman, R. G.; Lian. Y. J. Am. Chem. Soc. 2013, 135, 7122. (c) Wang, J.; He, J.; Zhi, C.; Luo, B.; Pan, 
Y.; Cao, X.; Gu, H. RSC Adv. 2014, 4, 16607. (d) Zhao, R.; Tan, Y.; Xie, Y.; Gao, C.; Liu, H.; Jiang, Y. Tetrahedron Lett. 2011, 52, 3805. (e) Kaiser, M.; Leitner, S. P.; Hirtenlehner, C.; List, M.; Gerisch, A.; Monkowius, U. Dalton Trans. 2013, 42, 14749. (f) Badjic, J. D.; Kostic, N. M. J. Mater. Chem. 2001, 11, 408. (g) Koduri, N. D.; Scott, H.; Hileman, B.; Cox, J. D.; Coffin, M.; Glicksberg, L.; Hussaini, S. R. Org. Lett. 2012, 14, 440.

(2) Park, Y.; Park, K. T.; Kim, J. G.; Chang, S. J. Am. Chem. Soc. 2015, 137, 4534. 
8. ${ }^{1} \mathrm{H}$ and ${ }^{13} \mathrm{C}$ NMR spectra

|<smiles>O=C(Nc1ccccc1N=Nc1ccccc1)c1ccccc1</smiles>
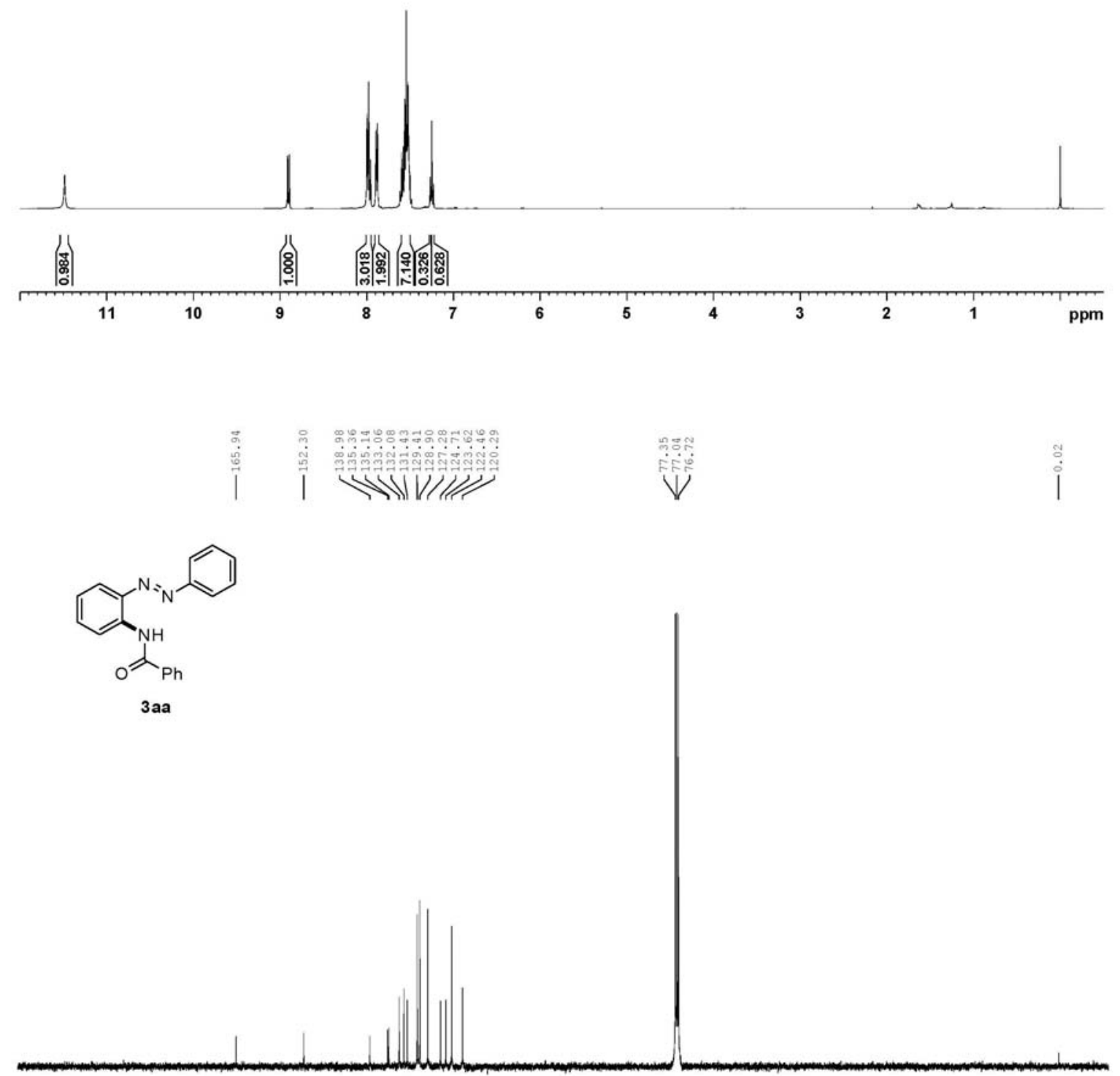

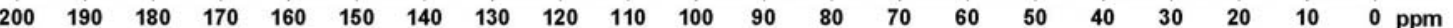




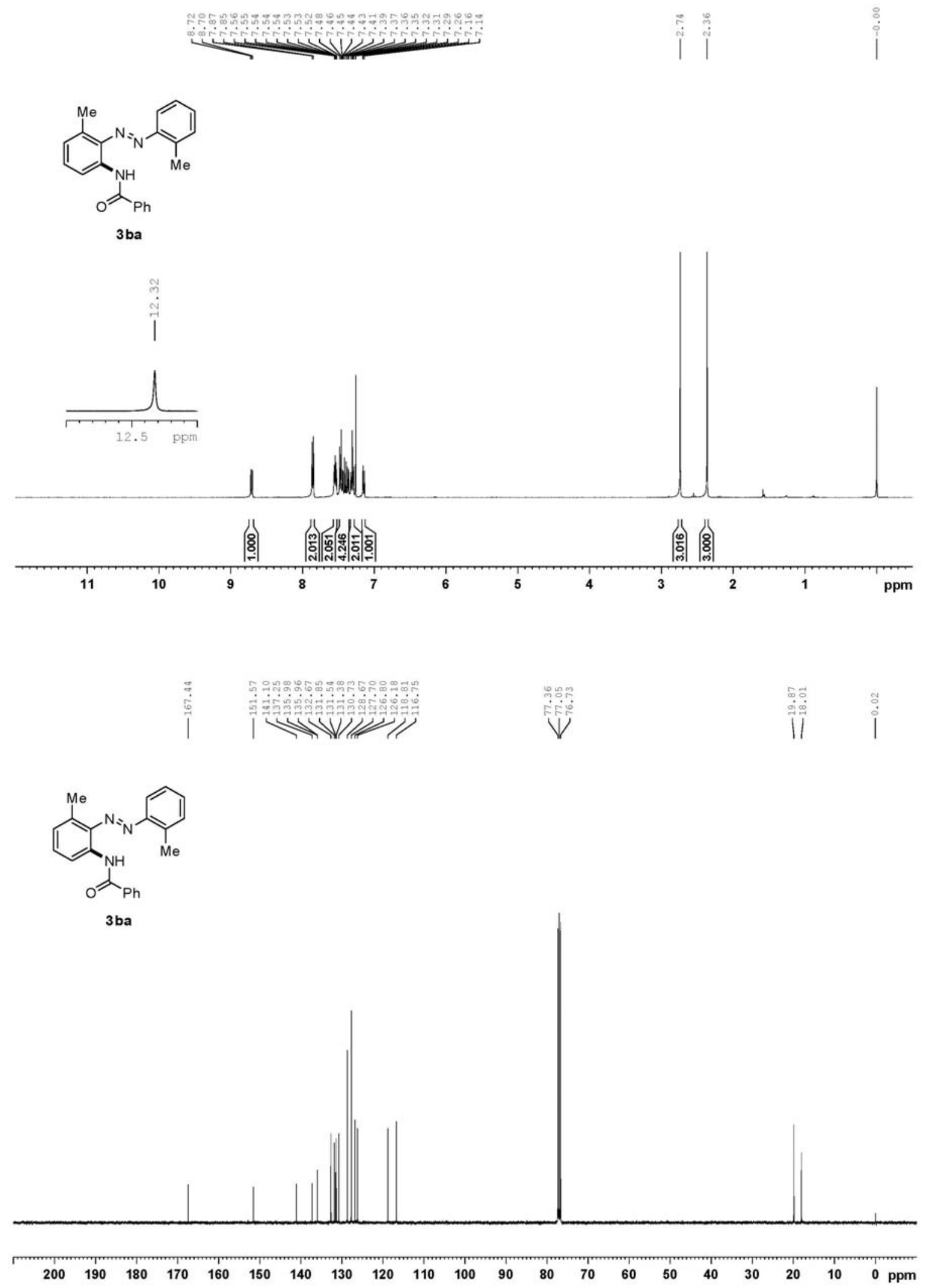


$\stackrel{?}{1}$

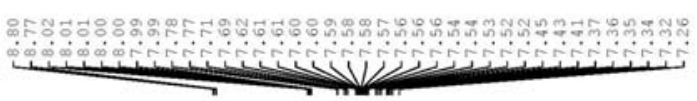

$\stackrel{9}{i=0}$

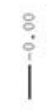
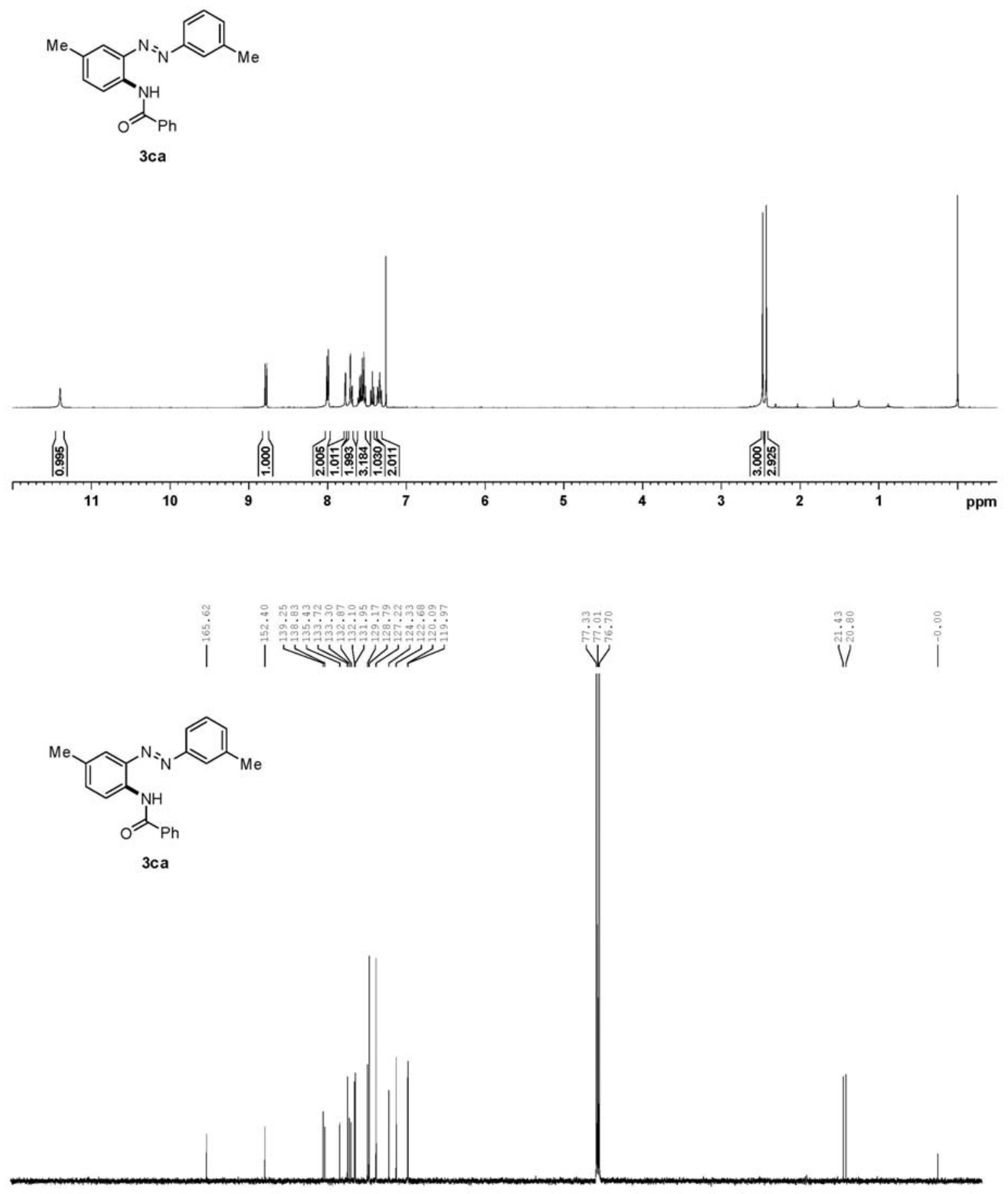

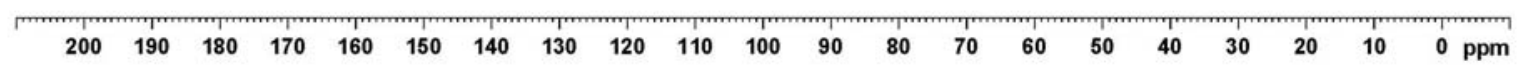

S28 

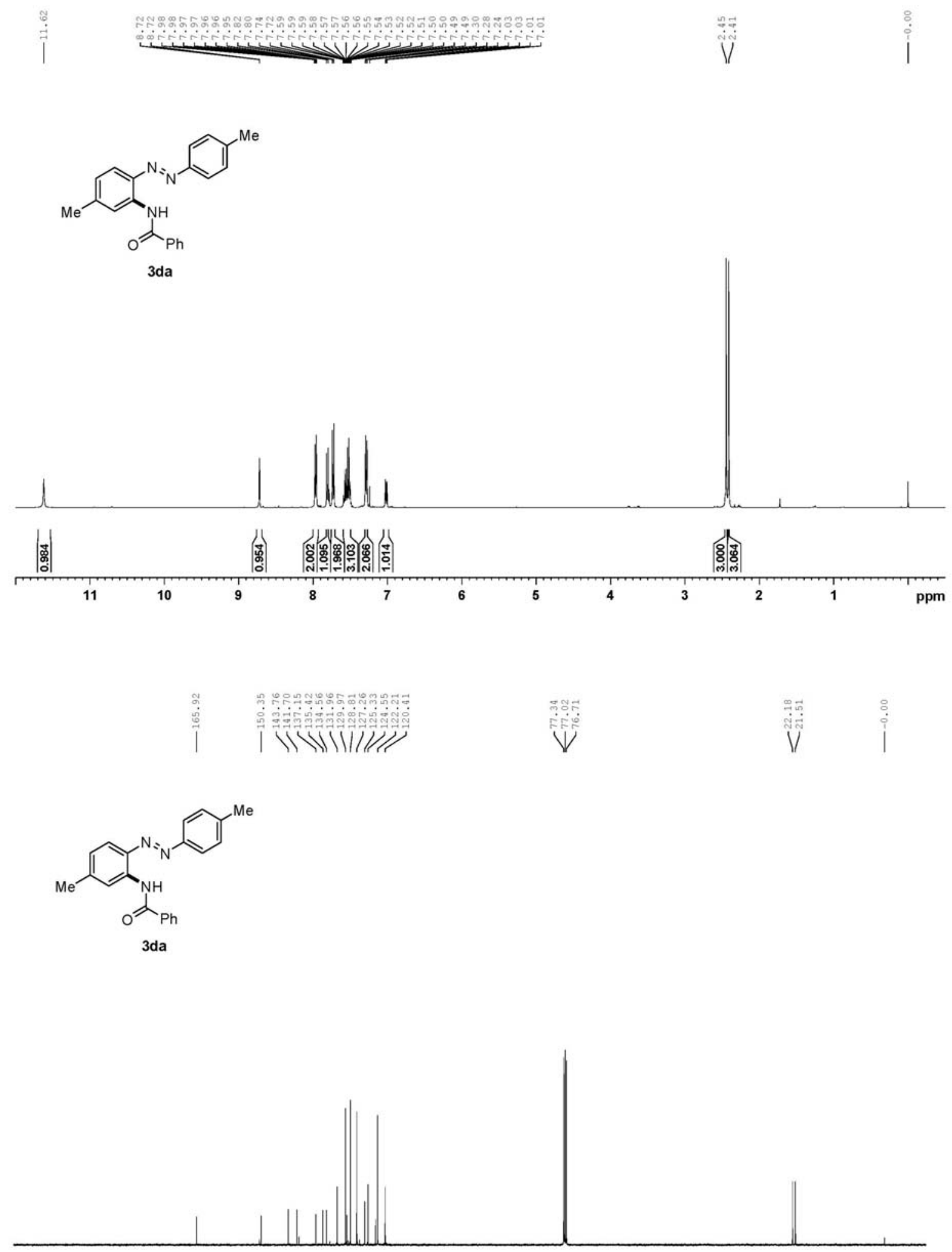

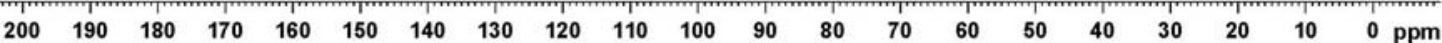




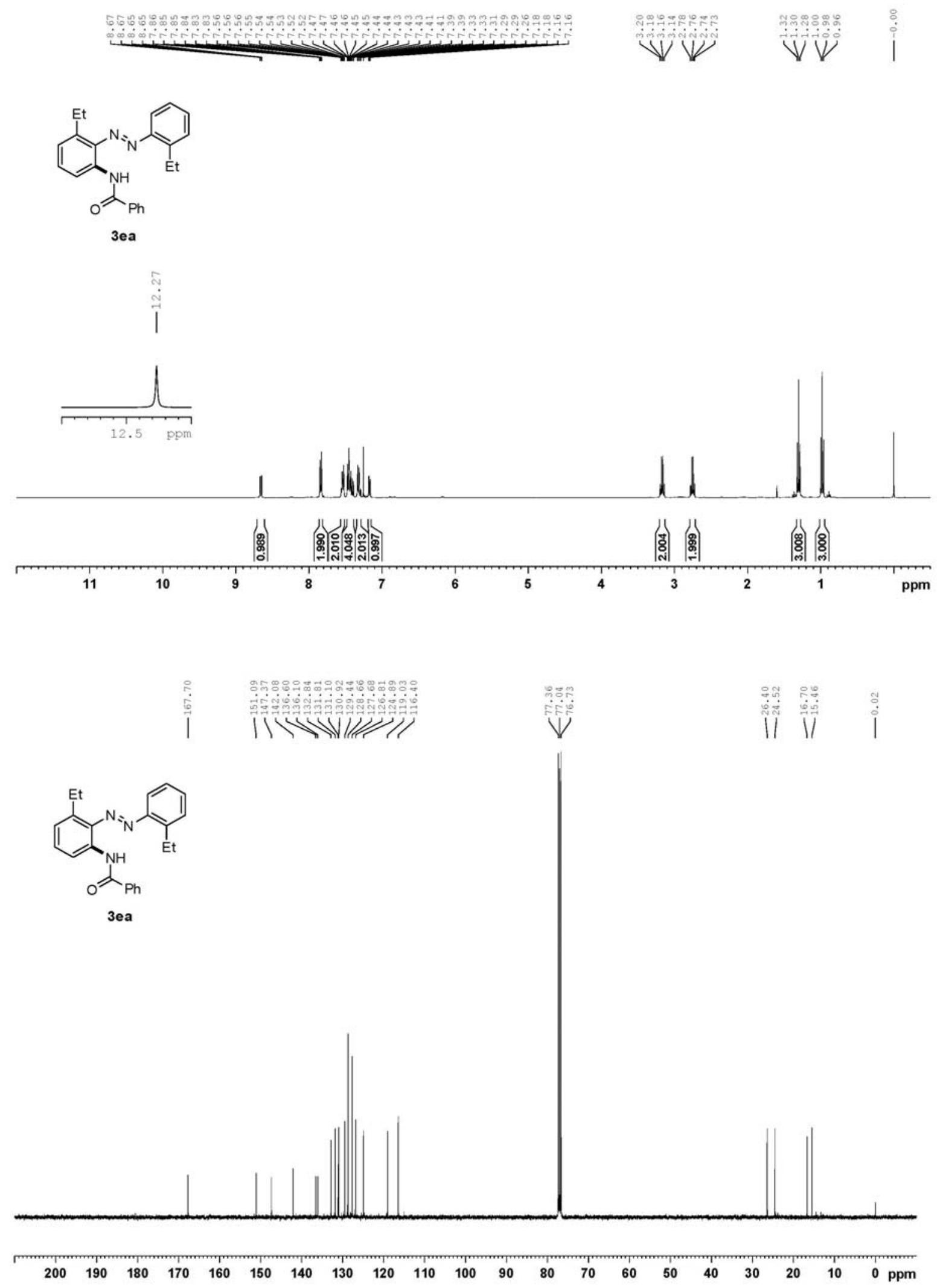




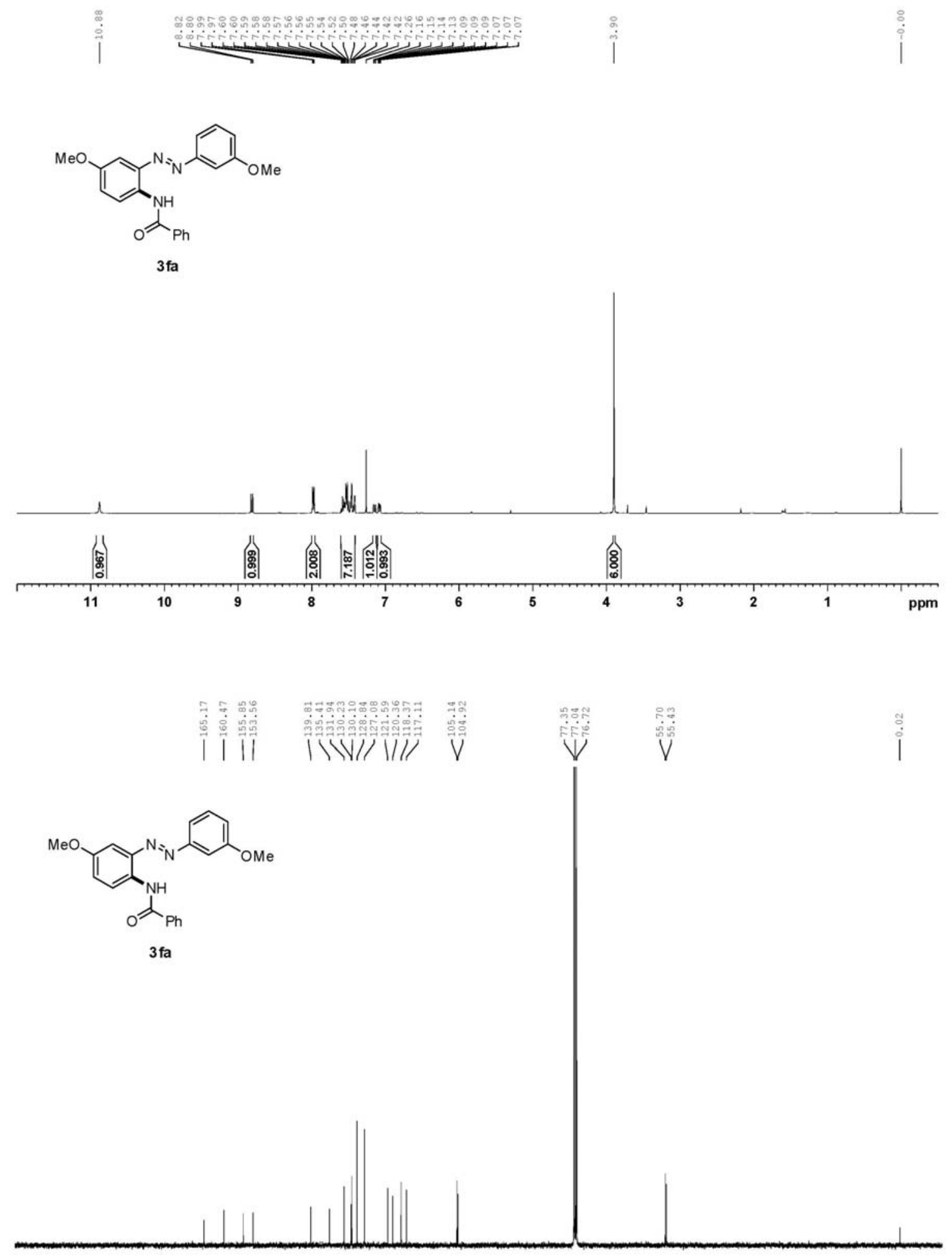

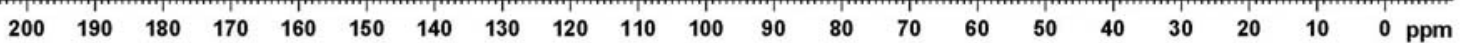




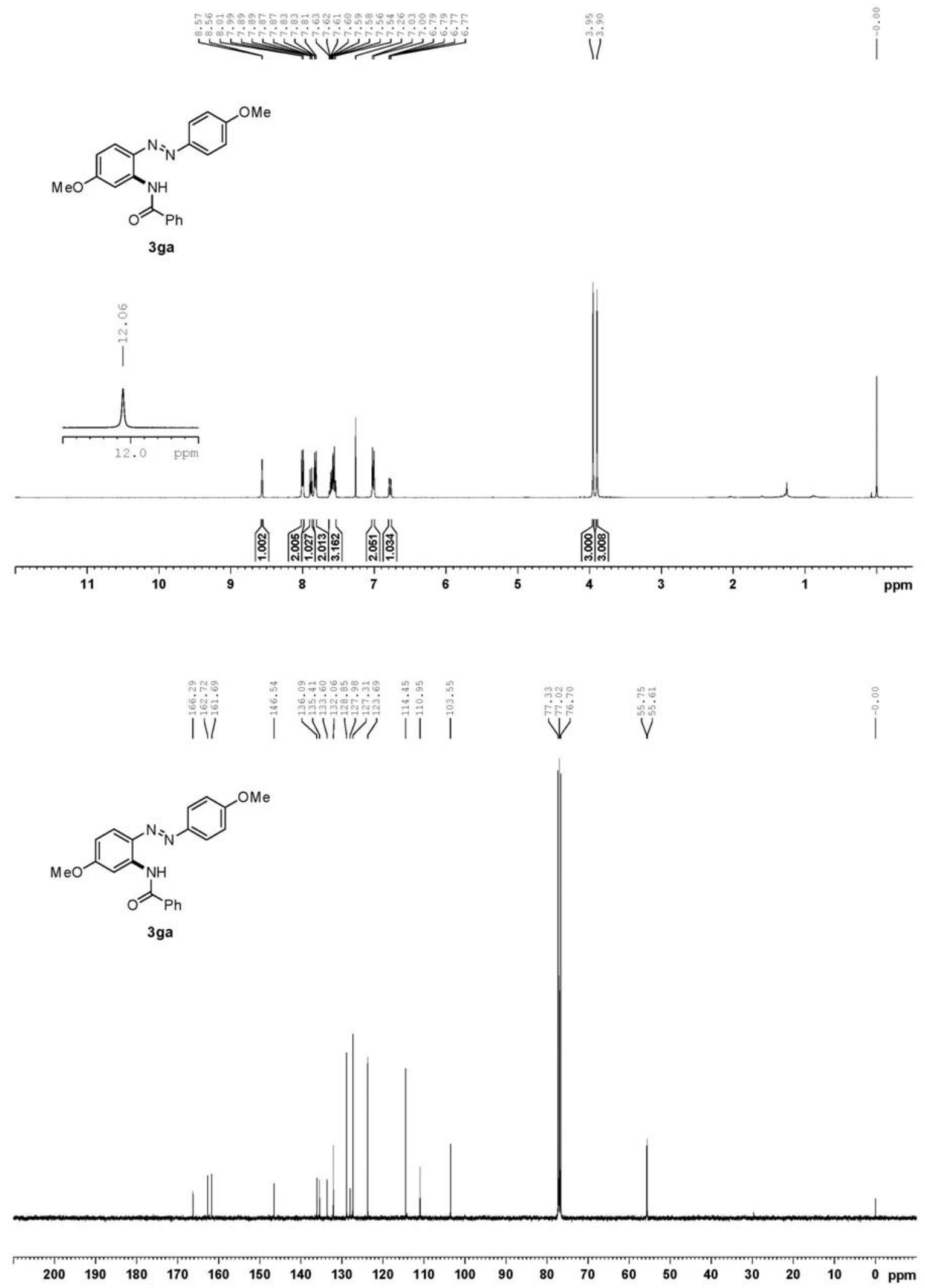




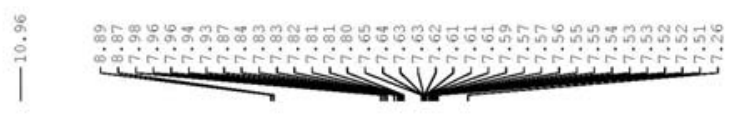

$\stackrel{\leftrightarrow}{\dot{0}}$
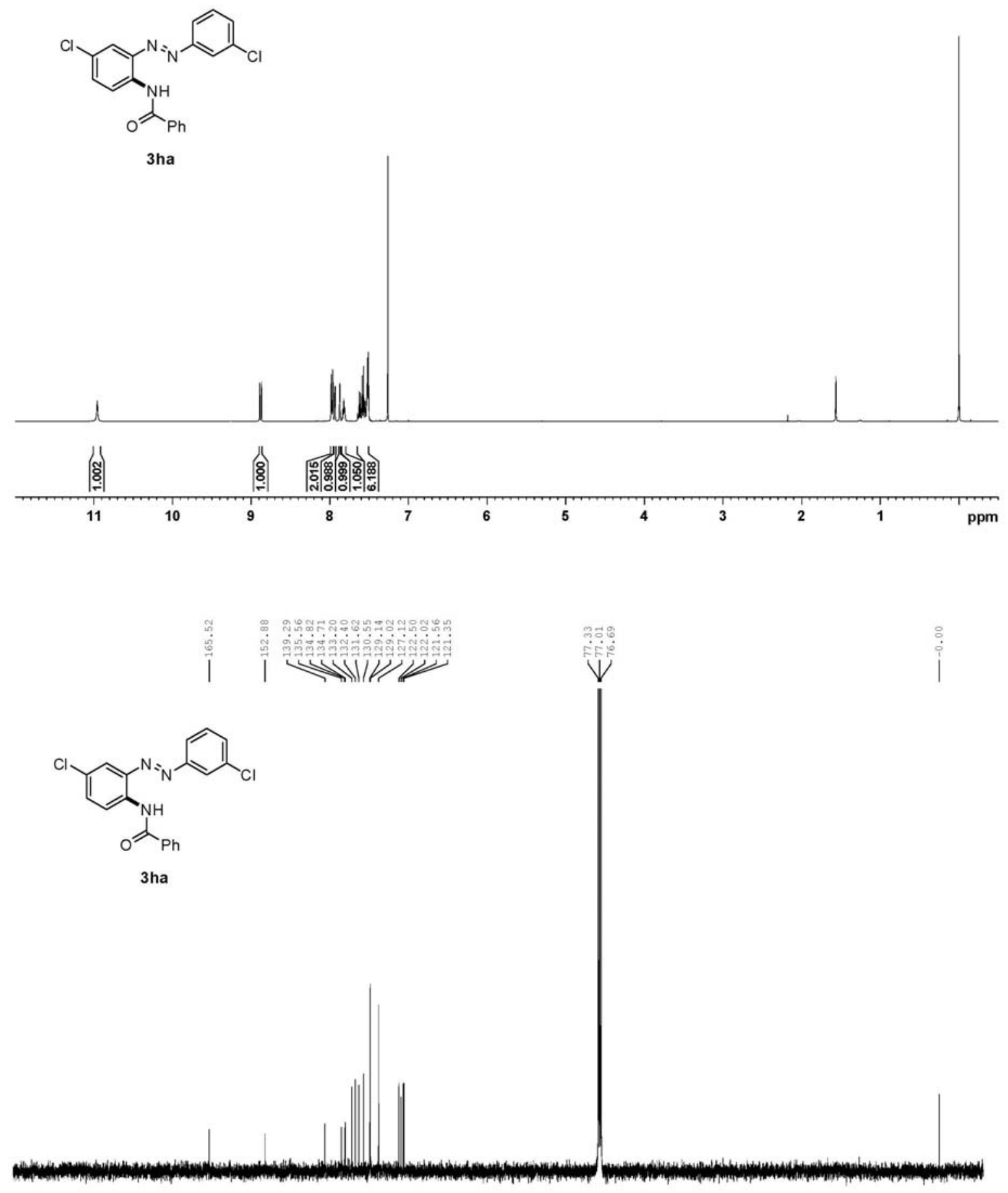

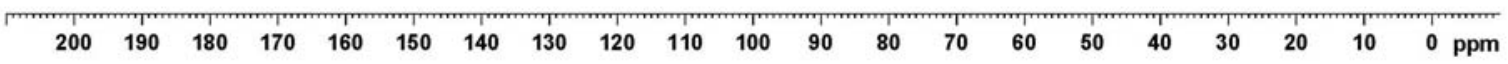

S33 

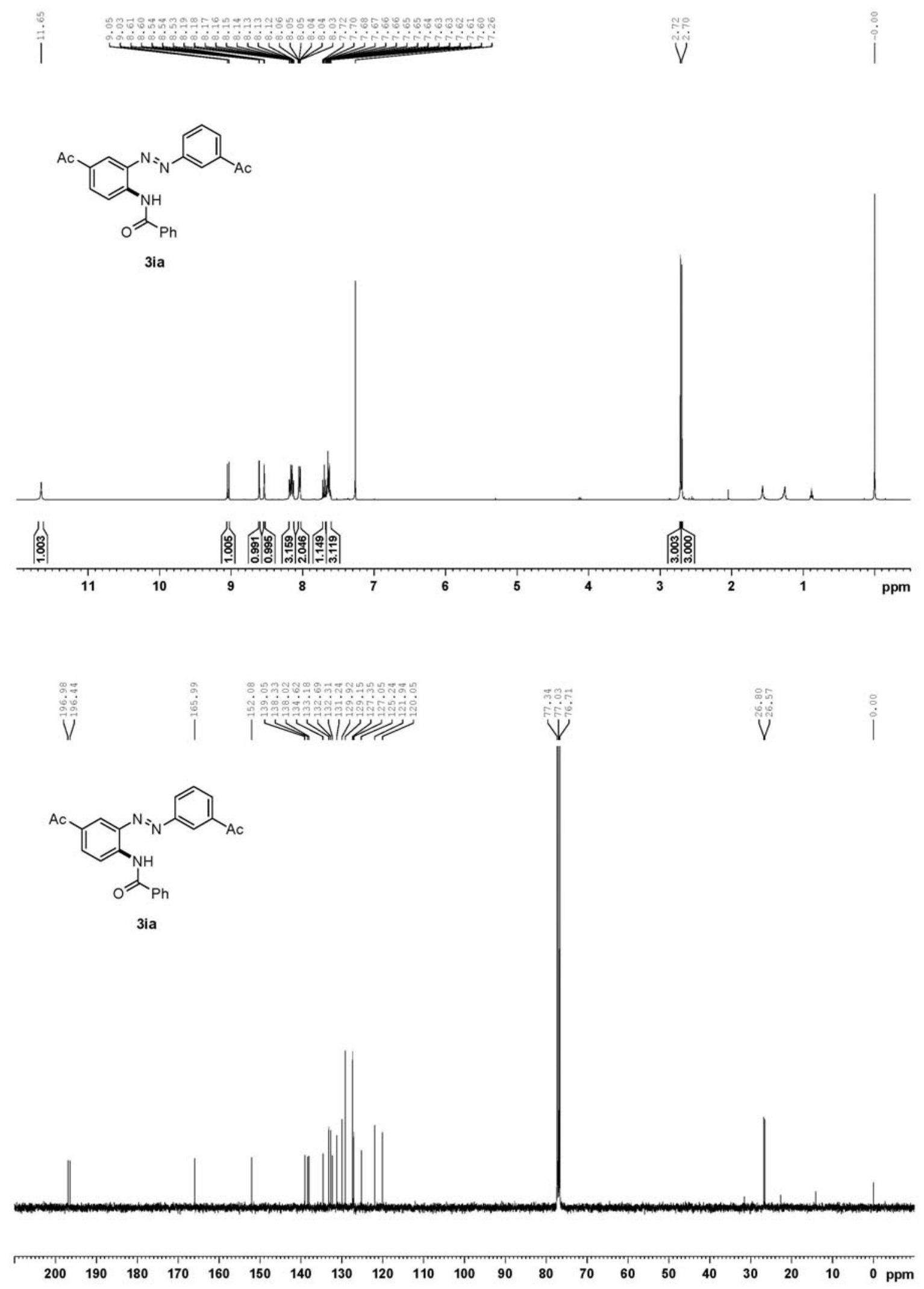

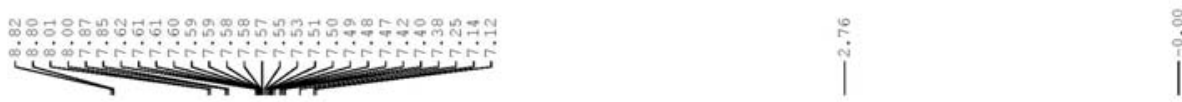<smiles>Cc1cccc(NC(=O)c2ccccc2)c1N=Nc1ccccc1</smiles>

3ja
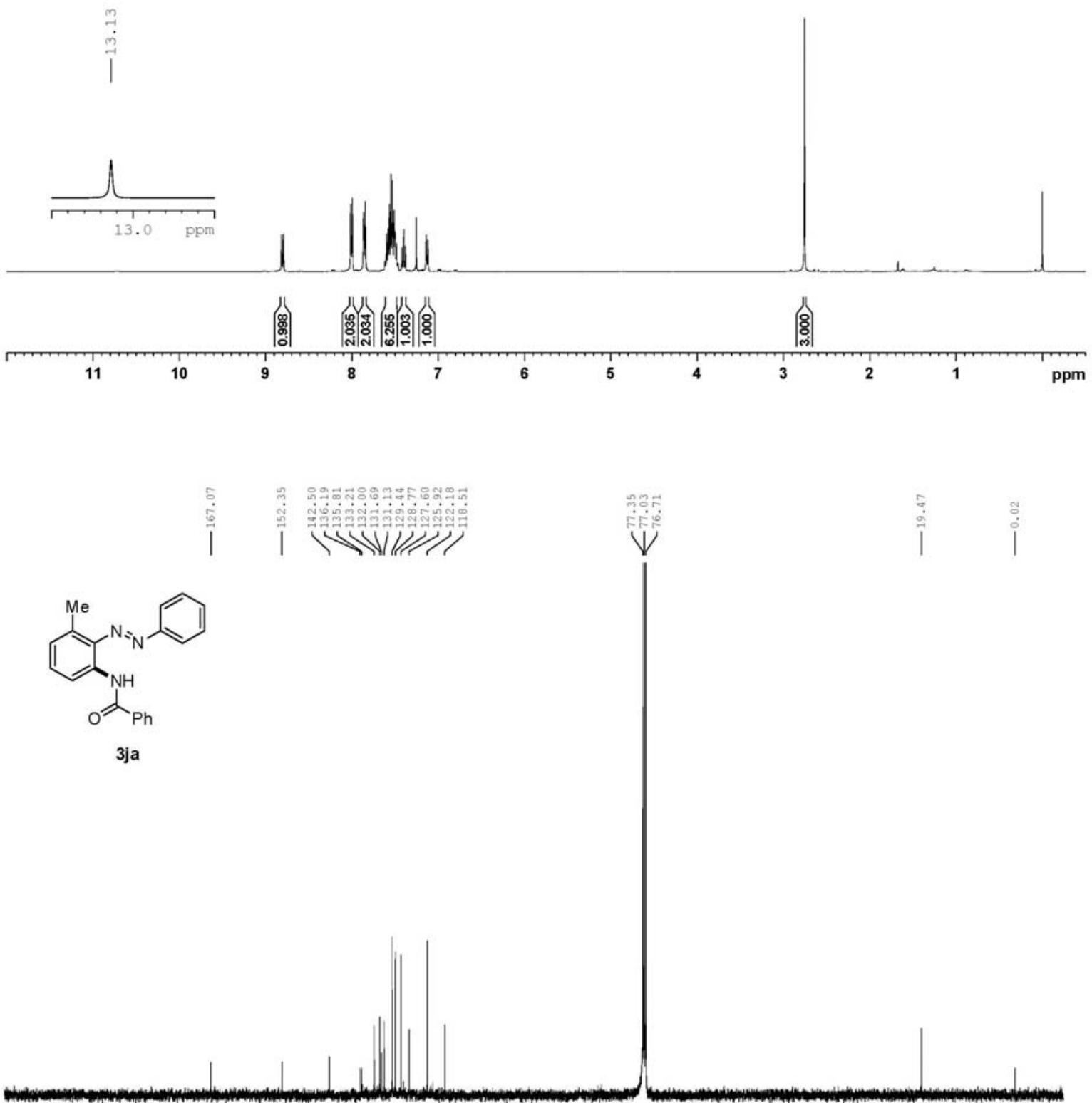

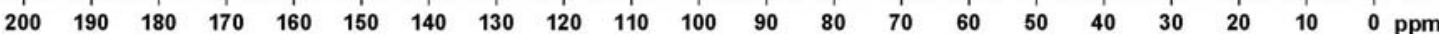




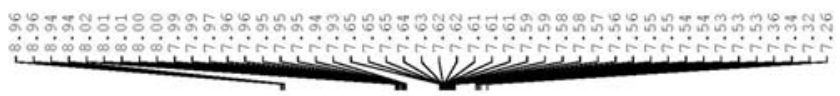

$\stackrel{\leftrightarrow}{\dot{0}}$
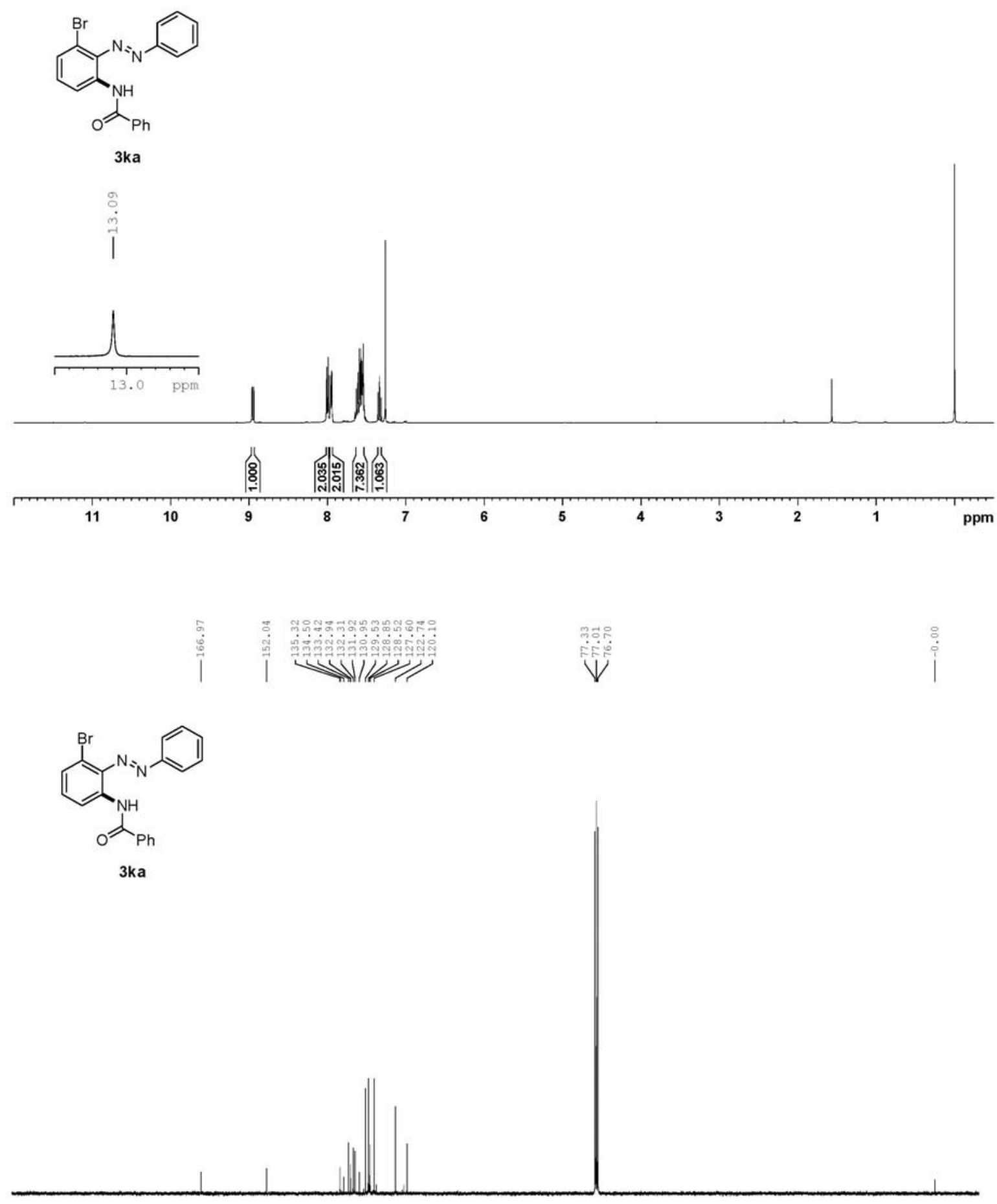

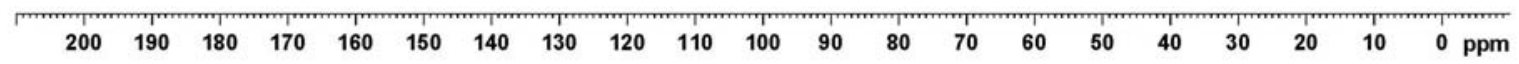

S36 
$\stackrel{\%}{\overrightarrow{1}}$

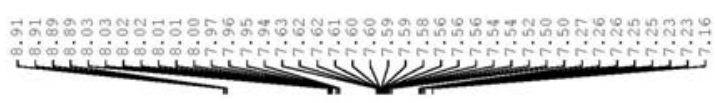

$\stackrel{\dddot{I}}{\tilde{I}}$

$\stackrel{\circ}{\grave{i}}$
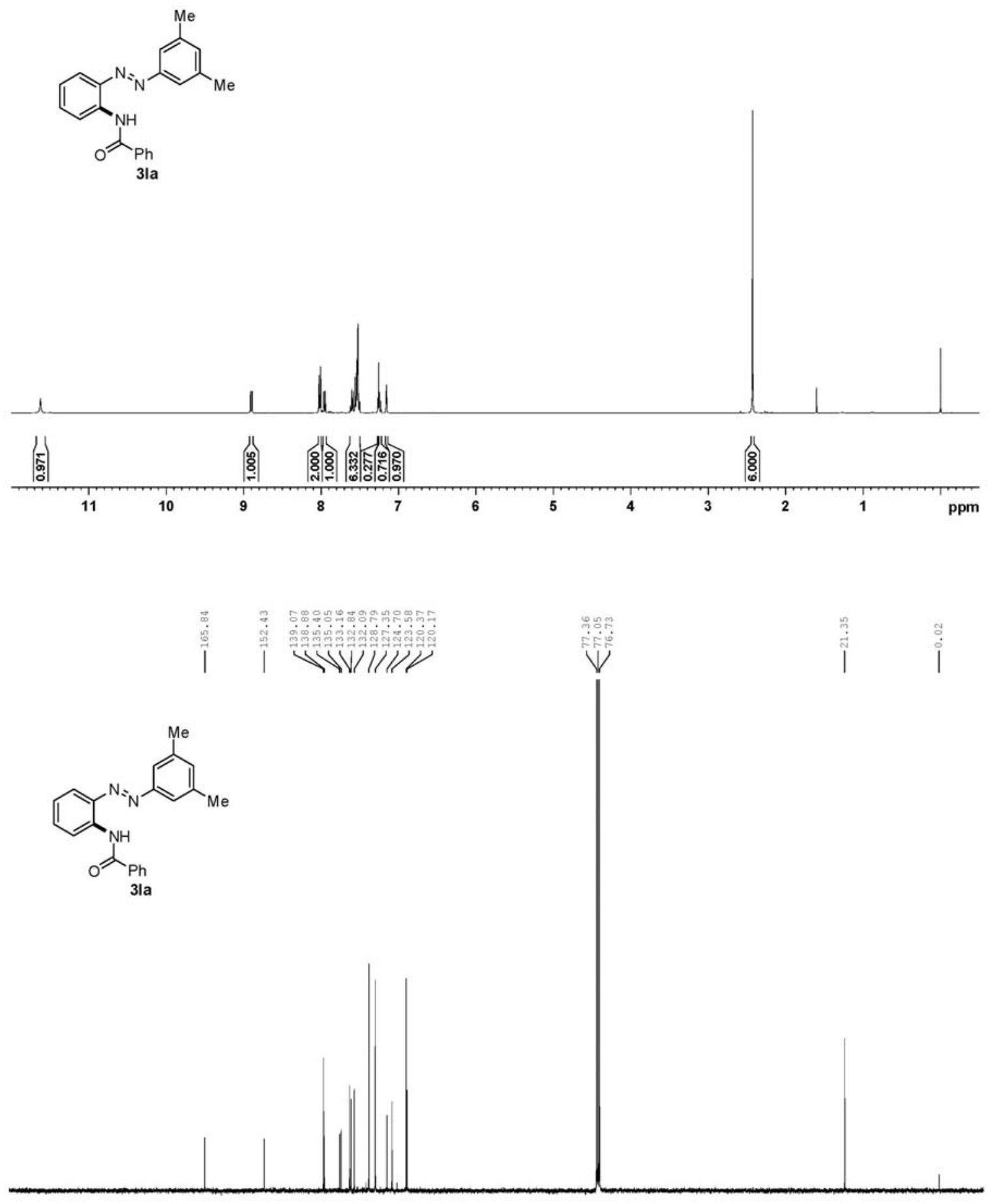

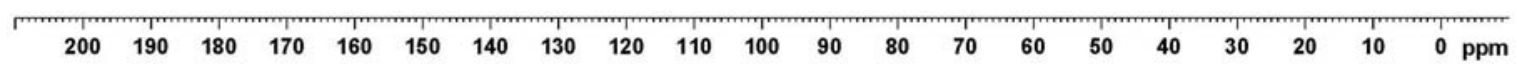

S37 


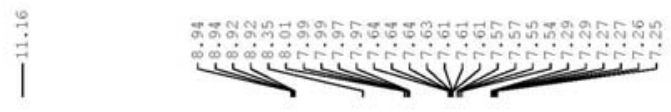<smiles>O=C(Nc1ccccc1N=Nc1cc(C(F)(F)F)cc(C(F)(F)F)c1)c1ccccc1</smiles>
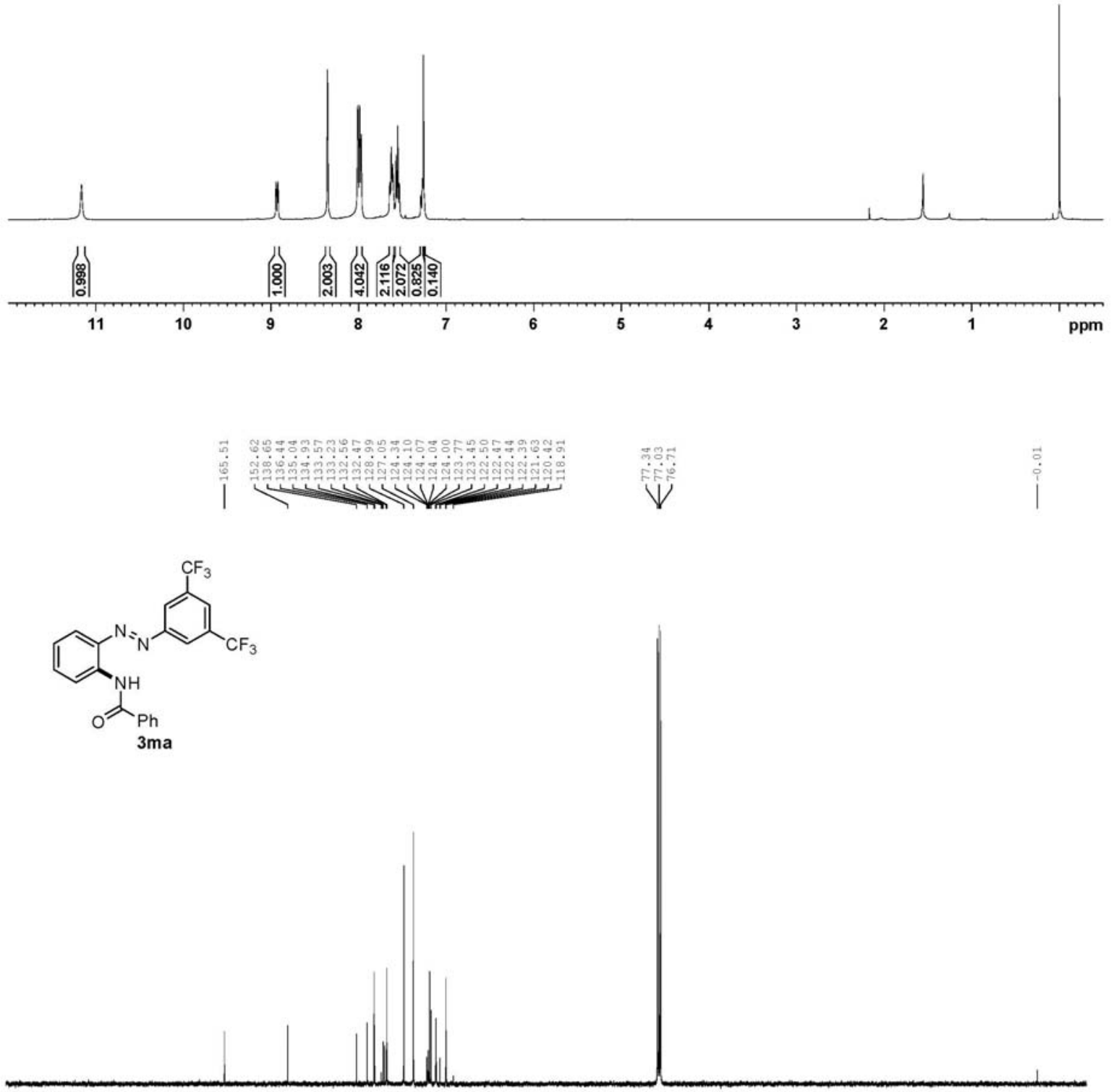

$\begin{array}{llllllllllllllllllllll}200 & 190 & 180 & 170 & 160 & 150 & 140 & 130 & 120 & 110 & 100 & 90 & 80 & 70 & 60 & 50 & 40 & 30 & 20 & 10 & 0 & \mathrm{ppm}\end{array}$ 
<smiles>O=C(Nc1ccccc1N=Nc1cc(C(F)(F)F)cc(C(F)(F)F)c1)c1ccccc1</smiles>
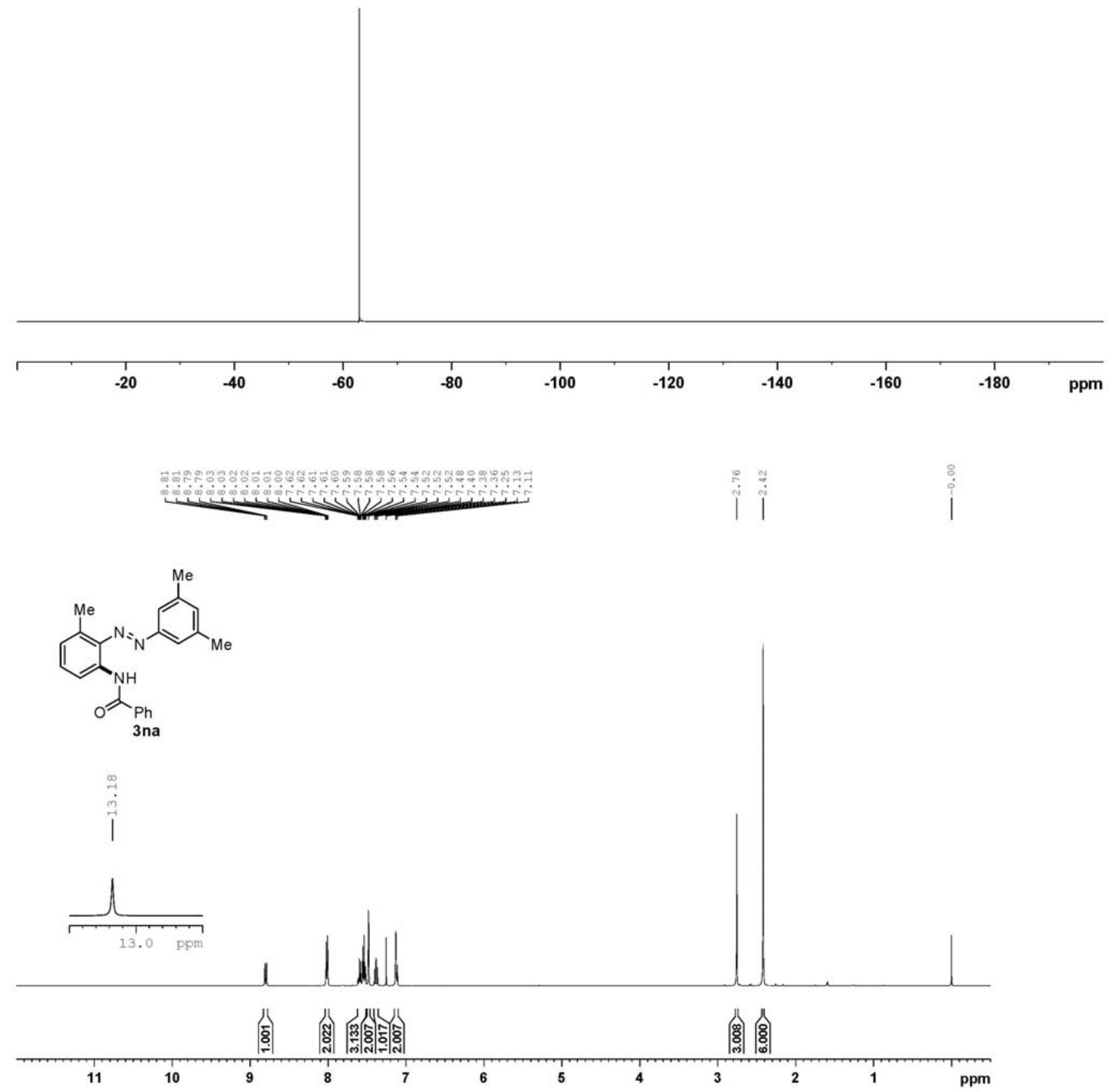


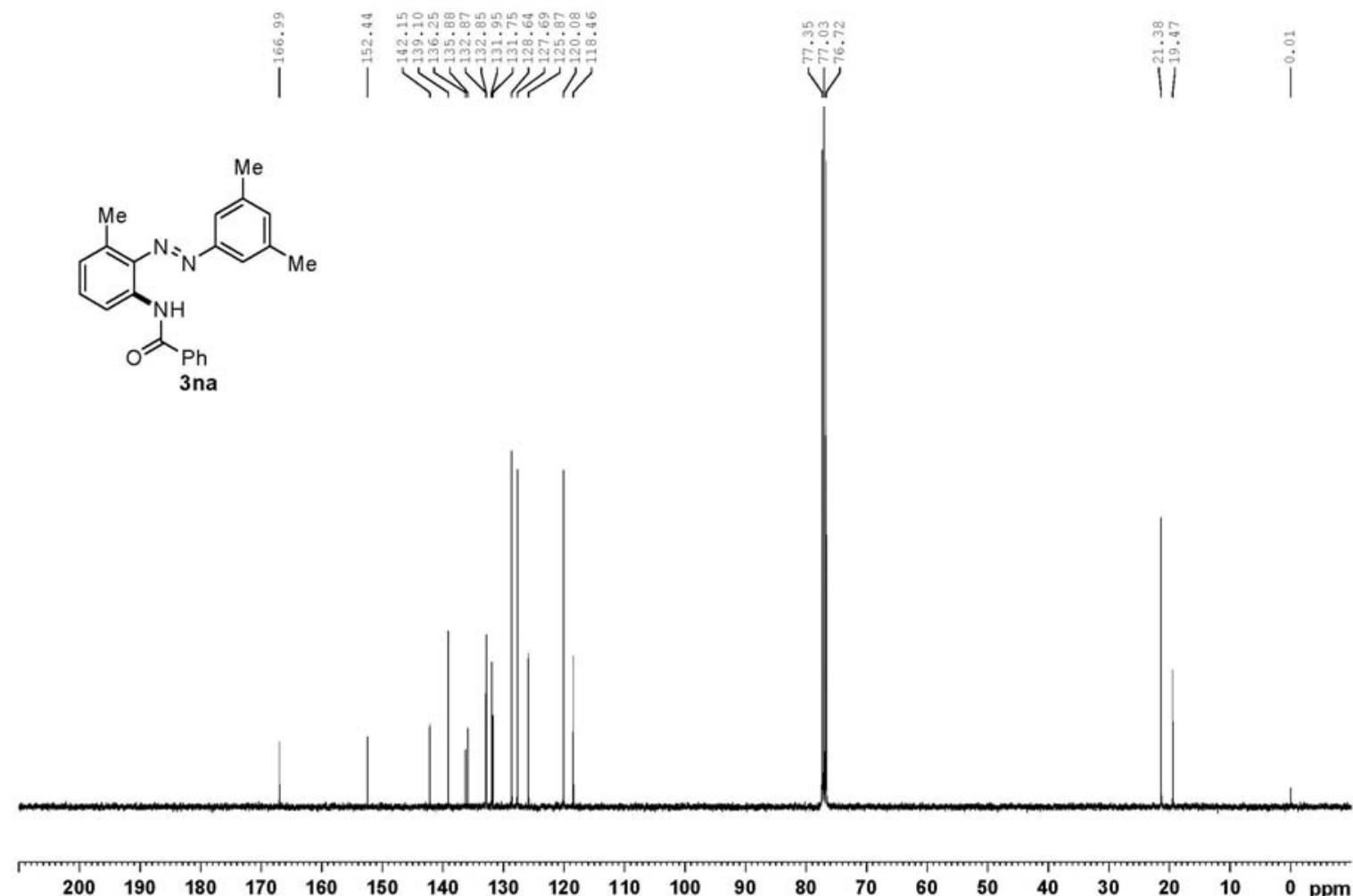

$\begin{array}{llllllllllllllllllllll}200 & 190 & 180 & 170 & 160 & 150 & 140 & 130 & 120 & 110 & 100 & 90 & 80 & 70 & 60 & 50 & 40 & 30 & 20 & 10 & 0 & \mathrm{ppm}\end{array}$
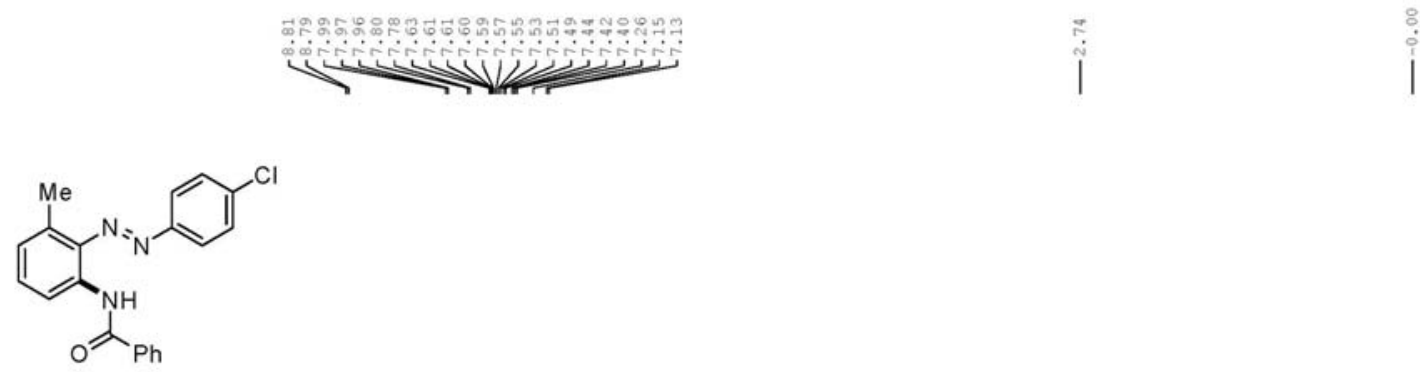

3oa

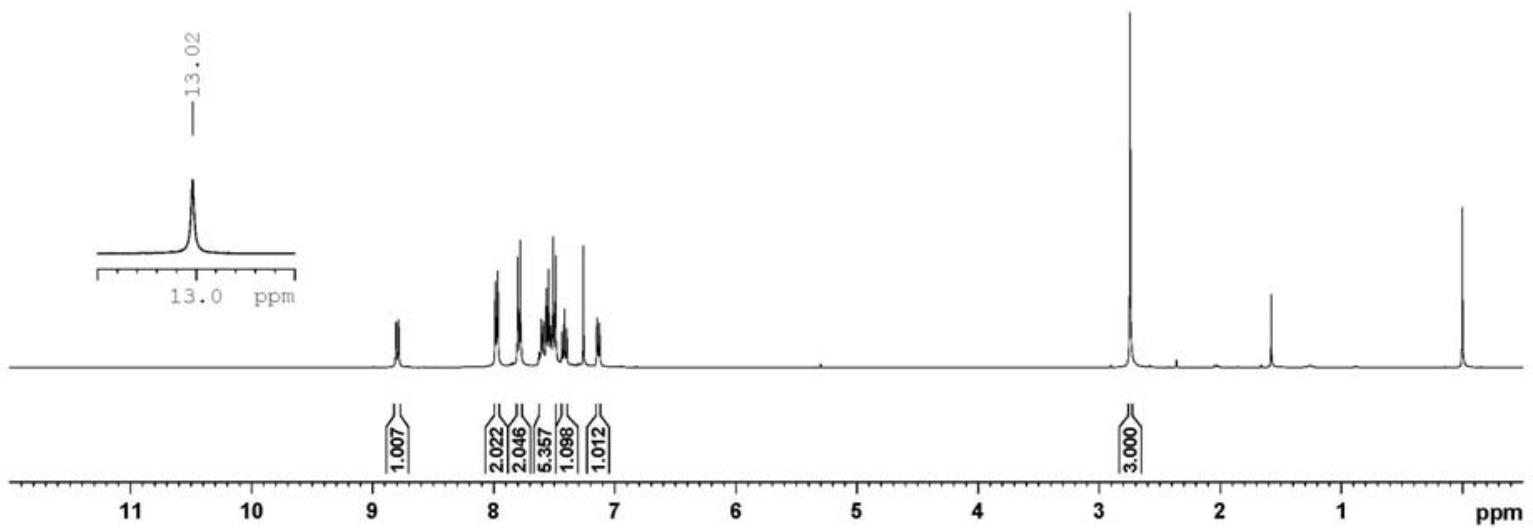




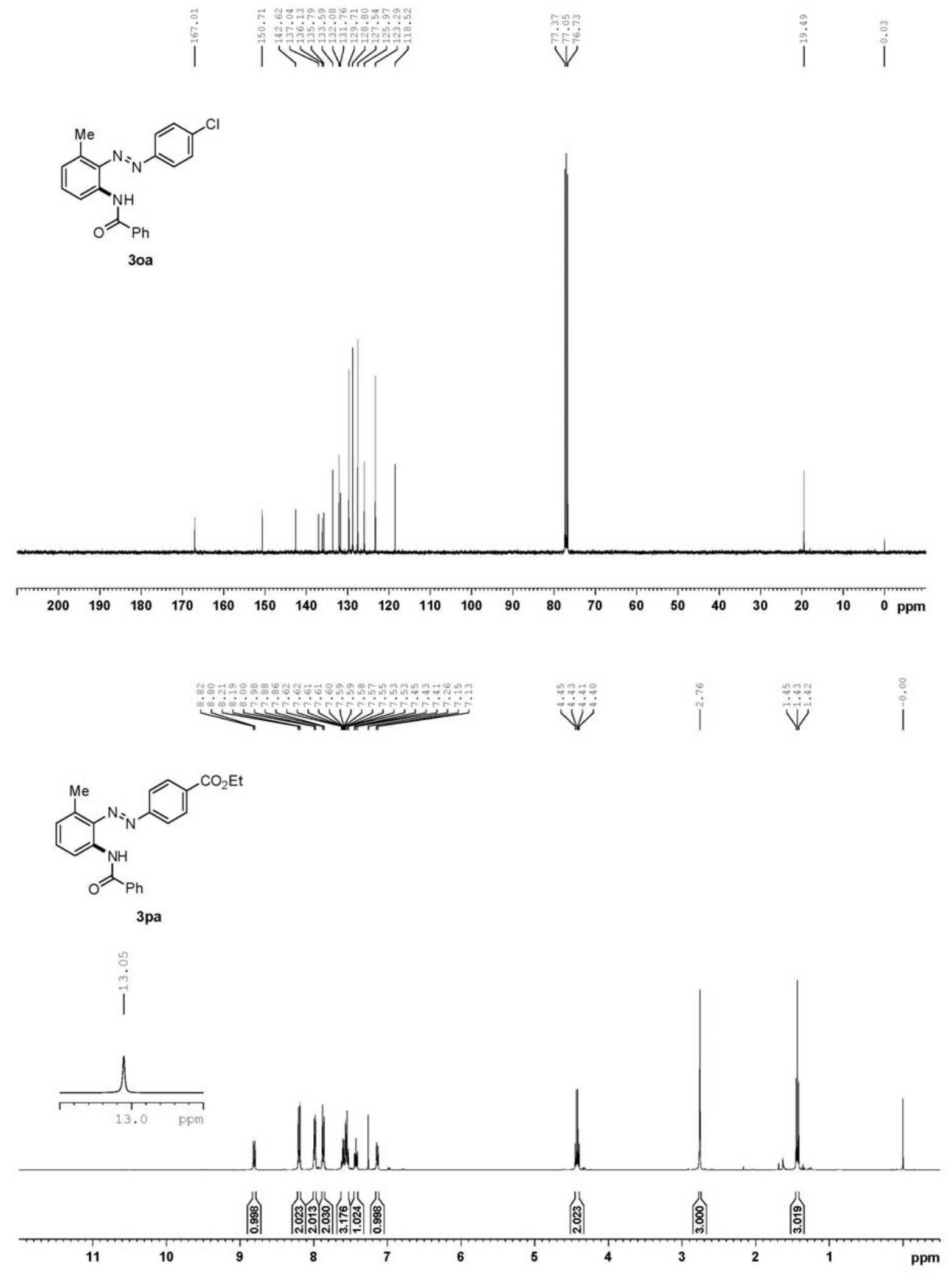




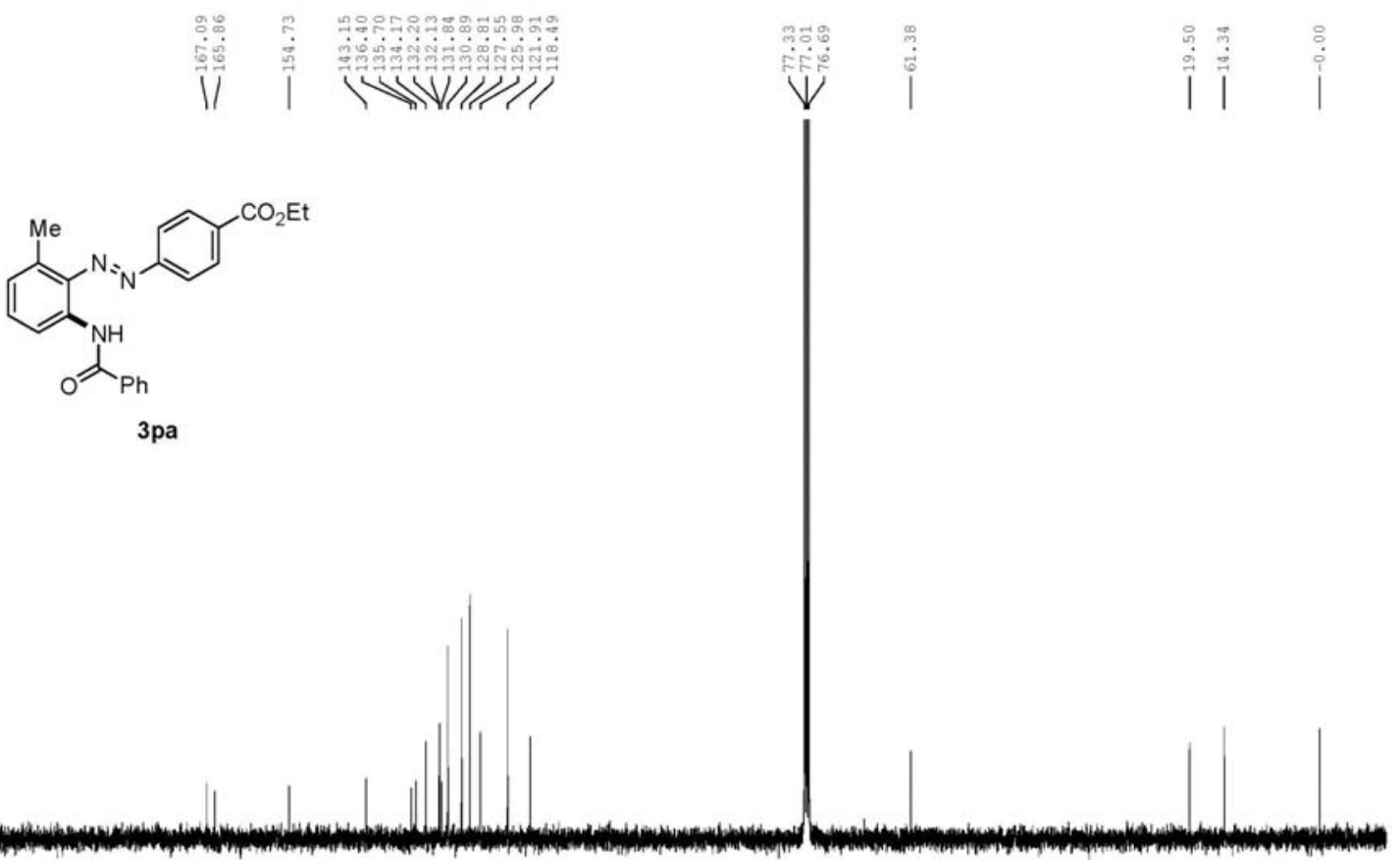

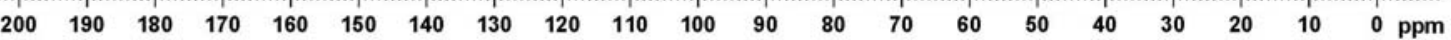
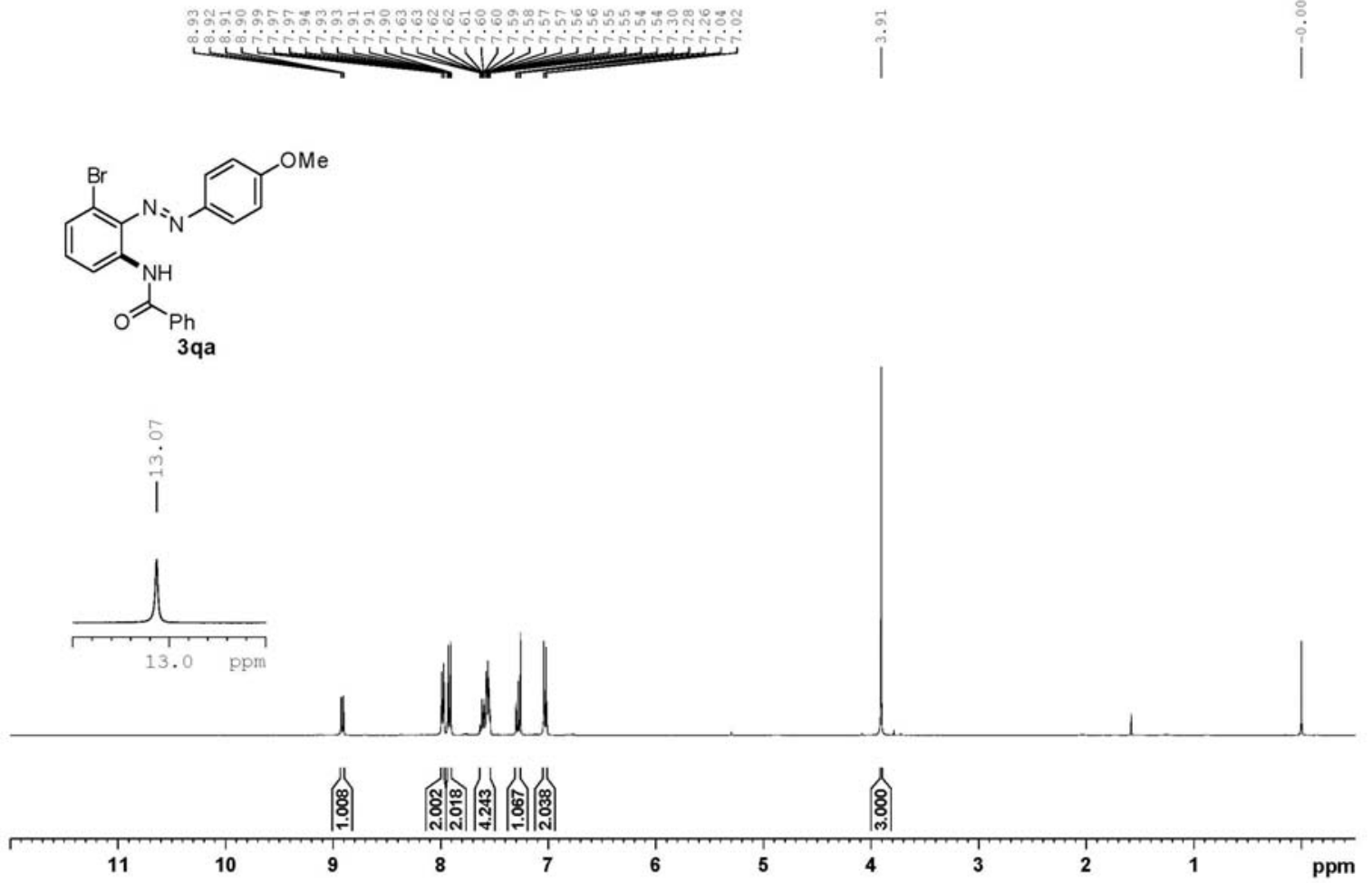


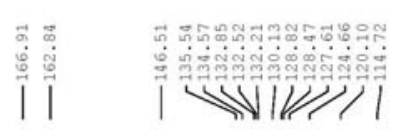

V

i
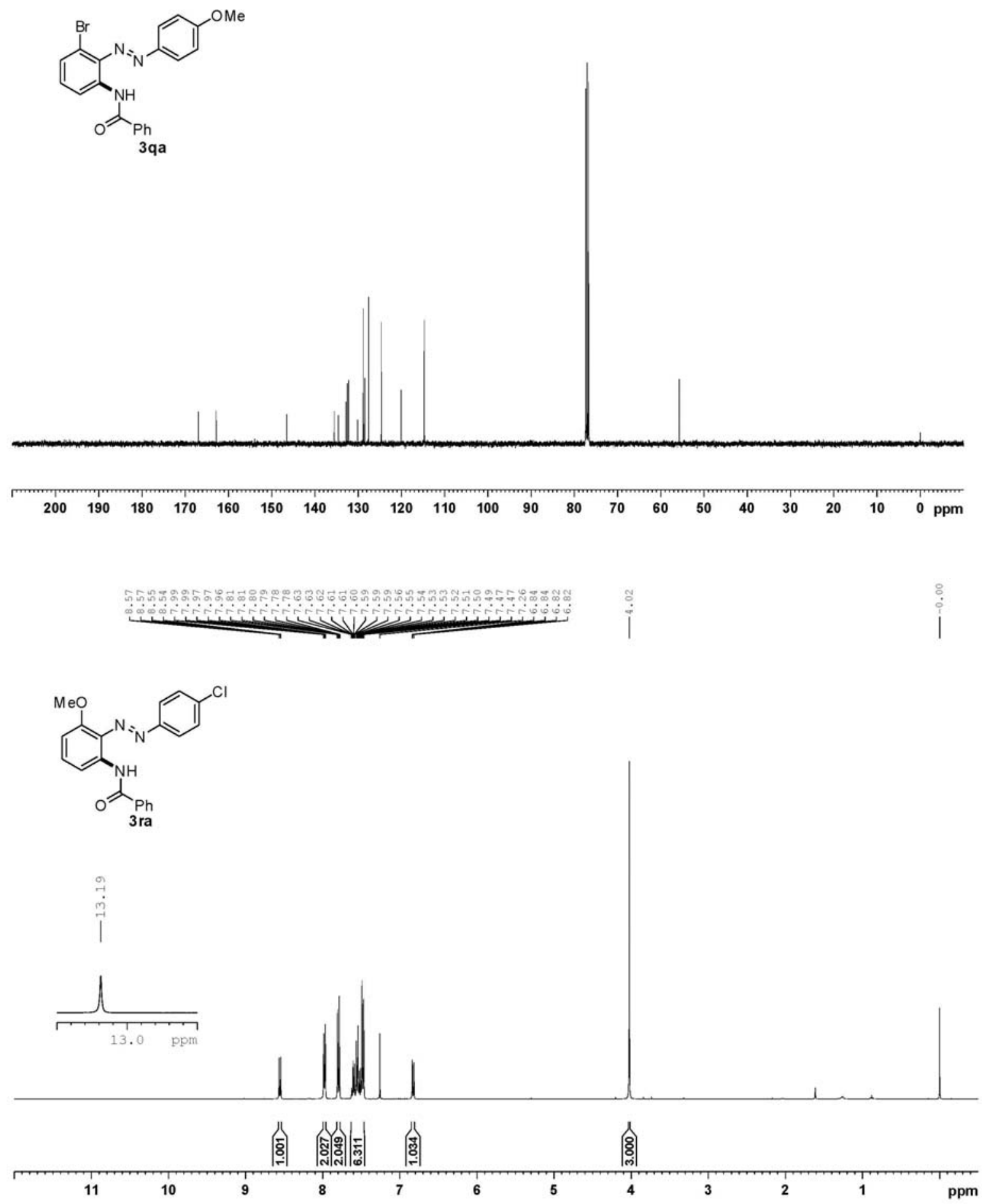

S43 


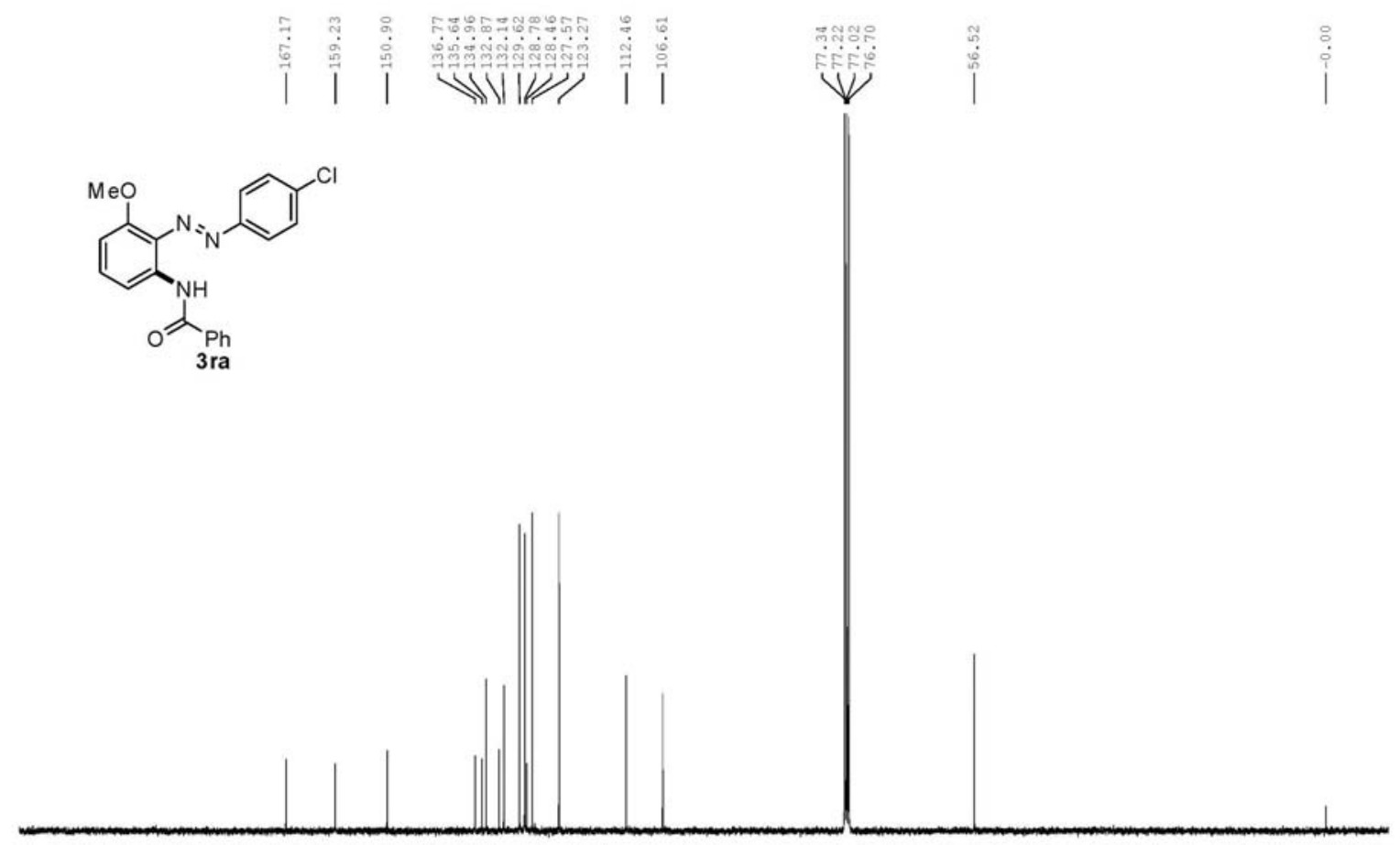

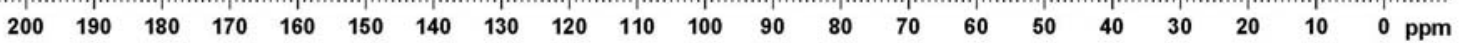
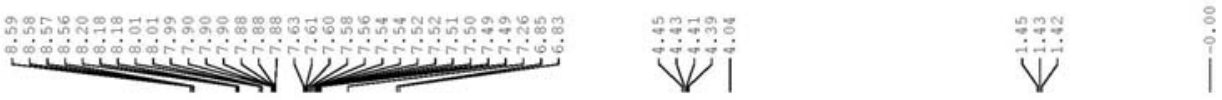

isa

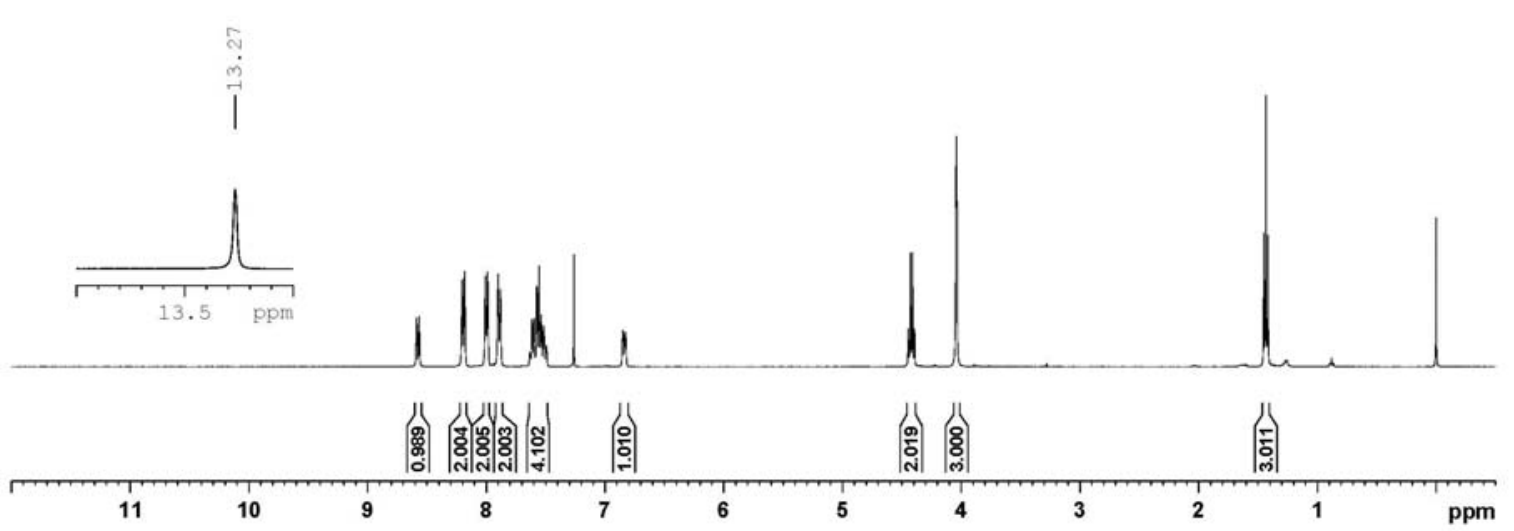




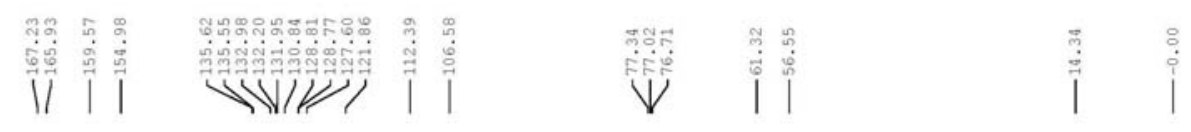
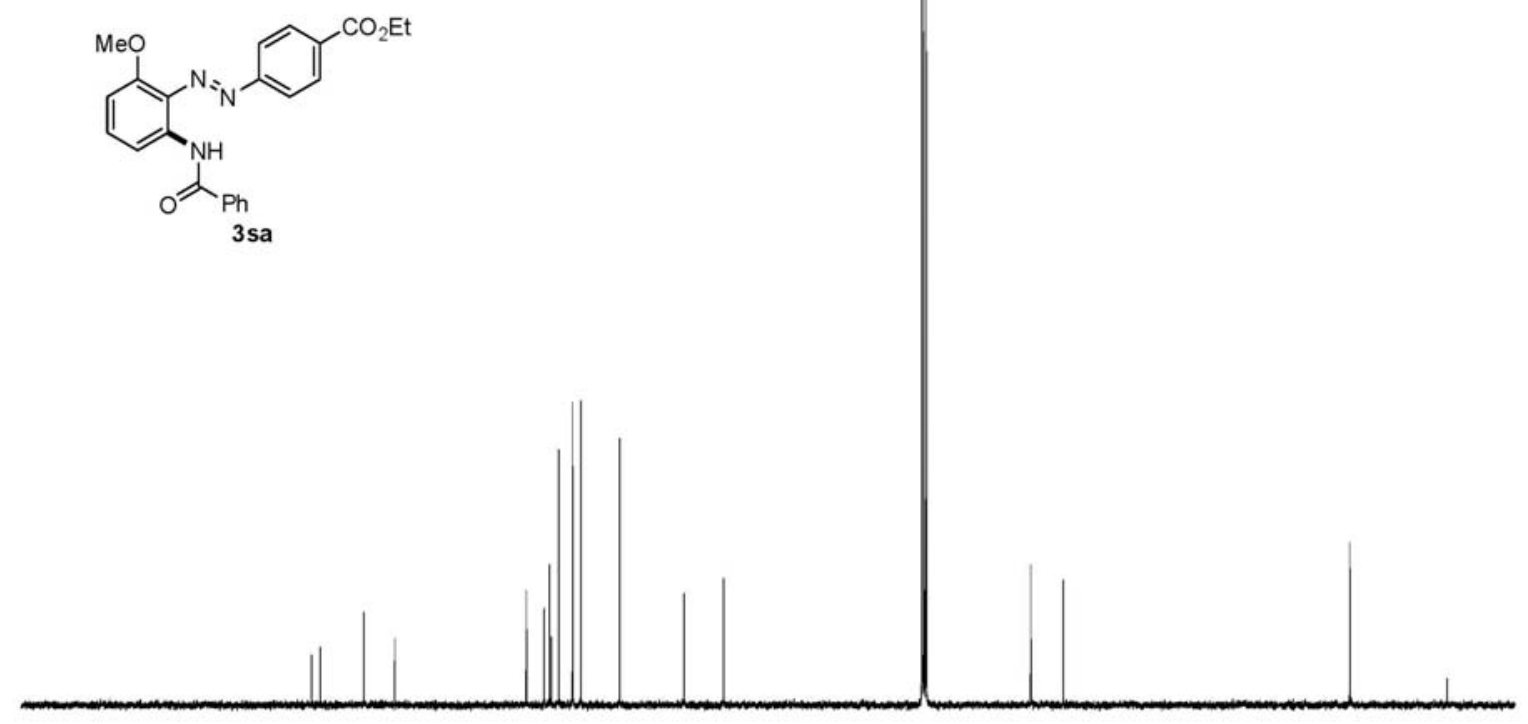

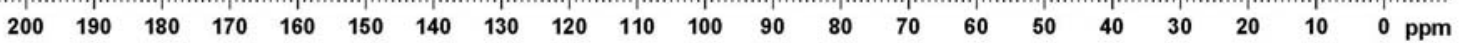

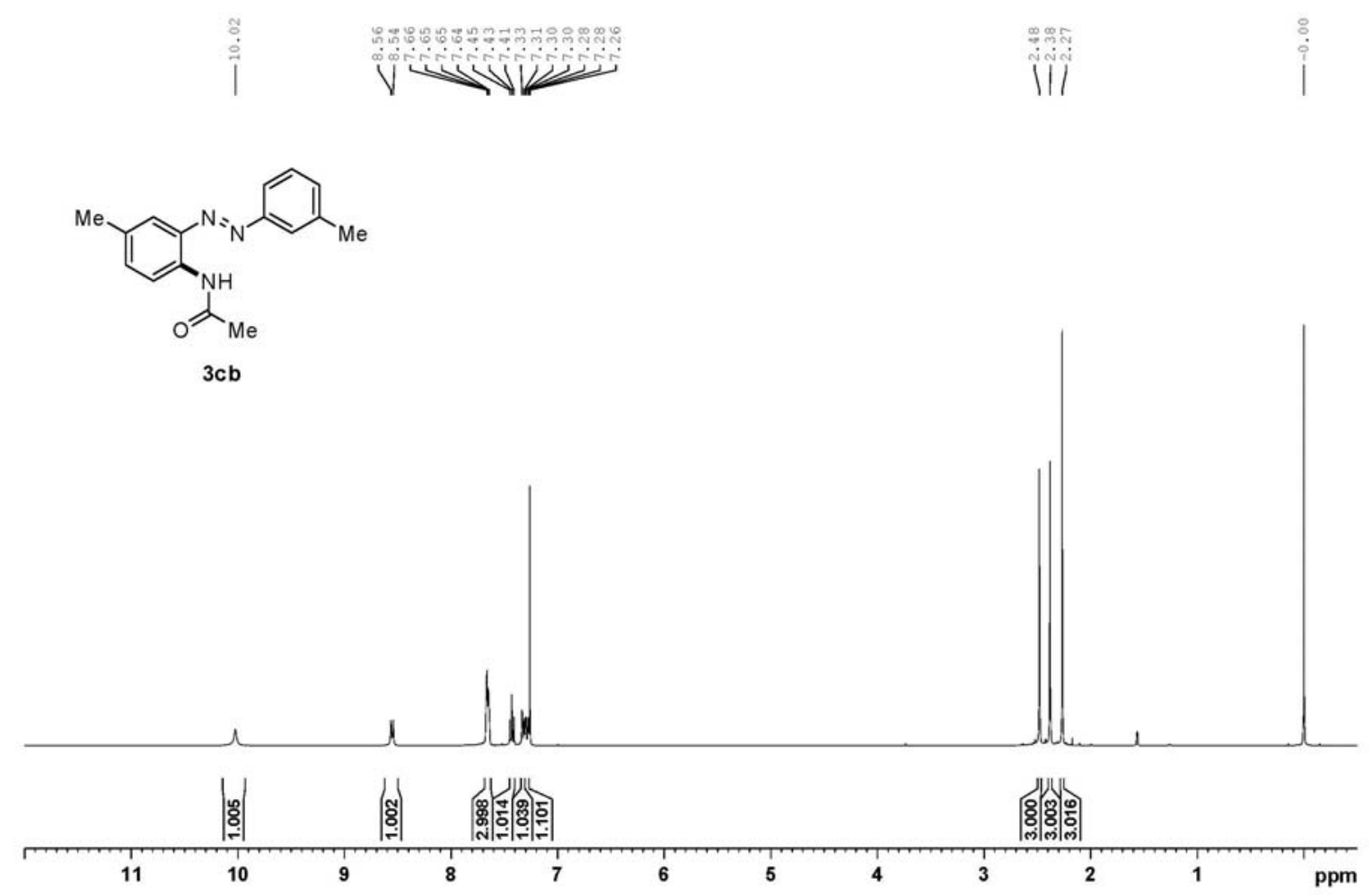




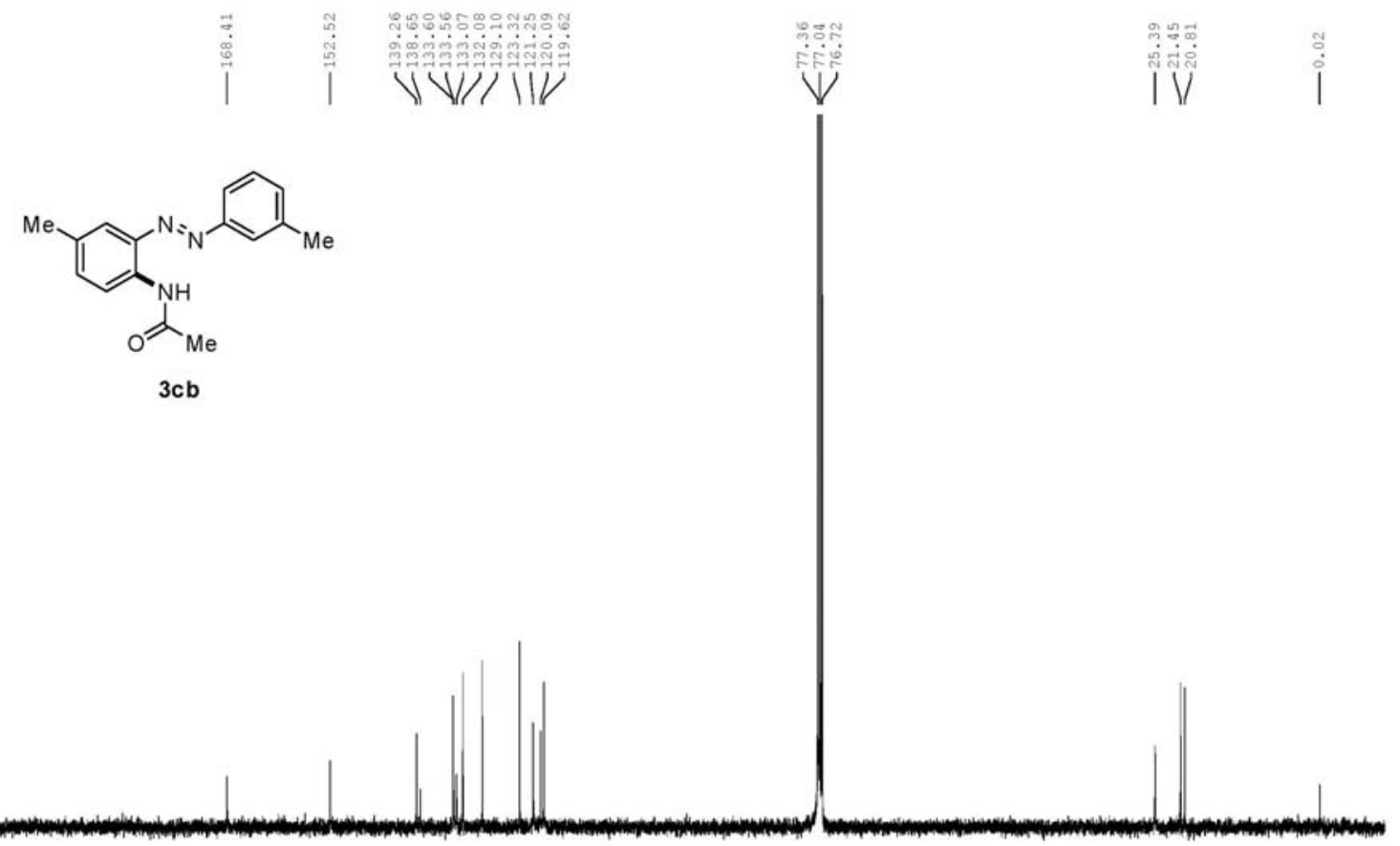

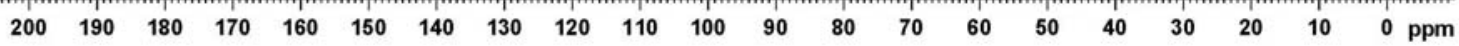
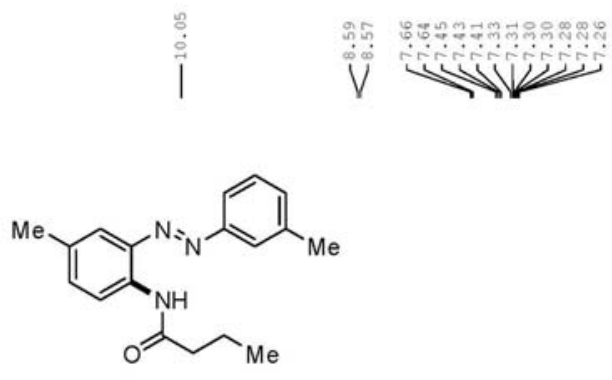

3cc

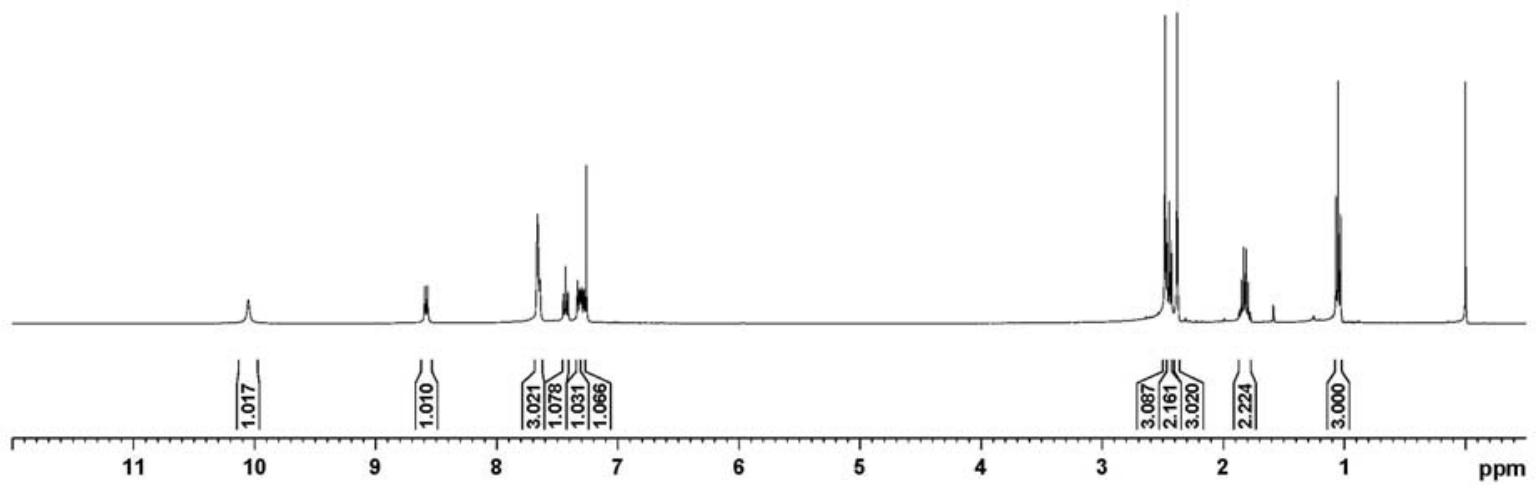




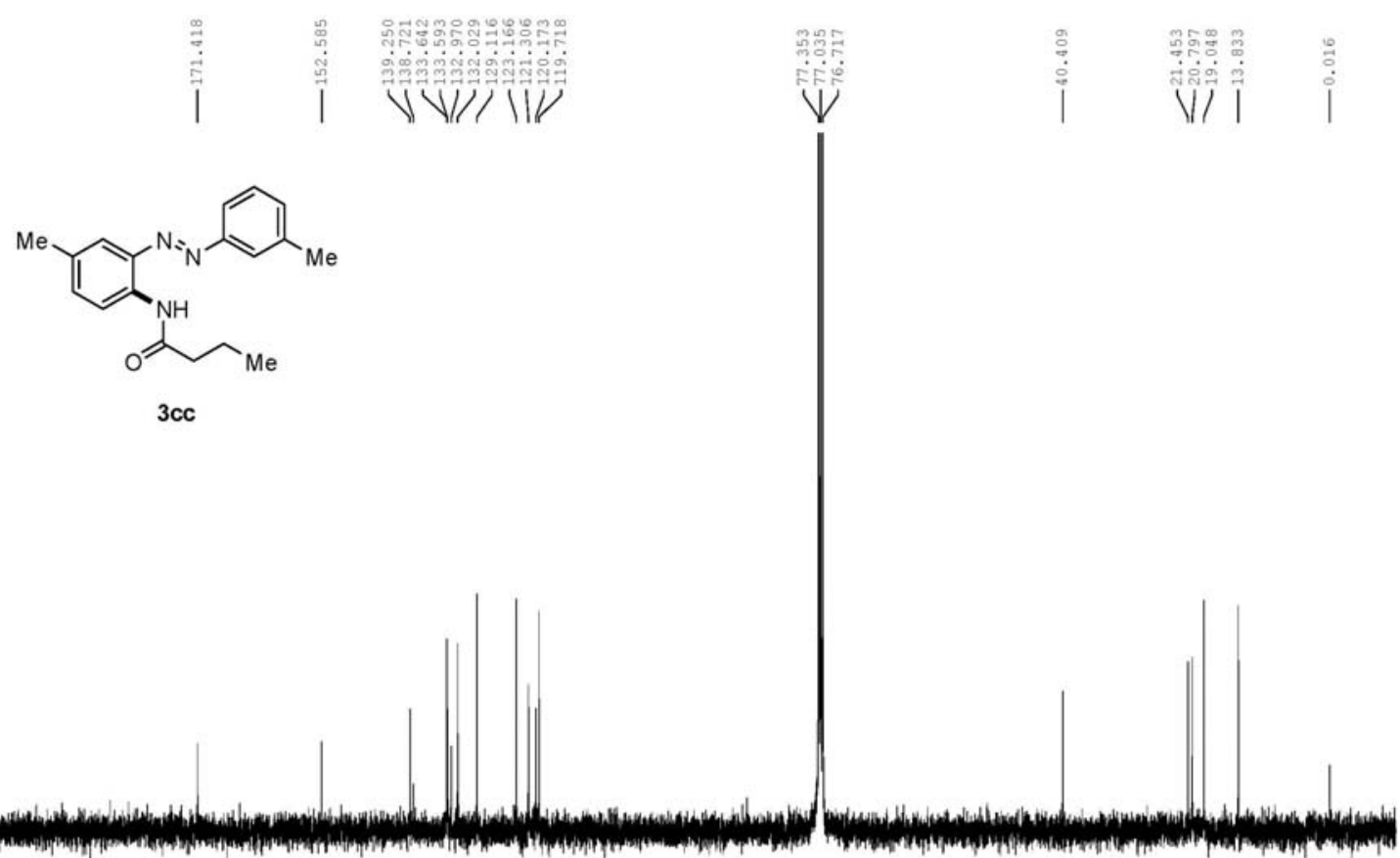

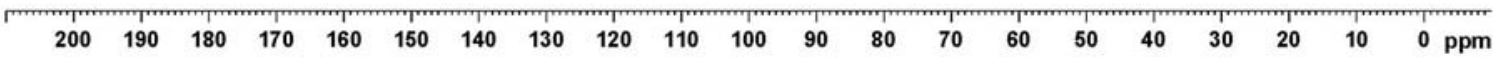
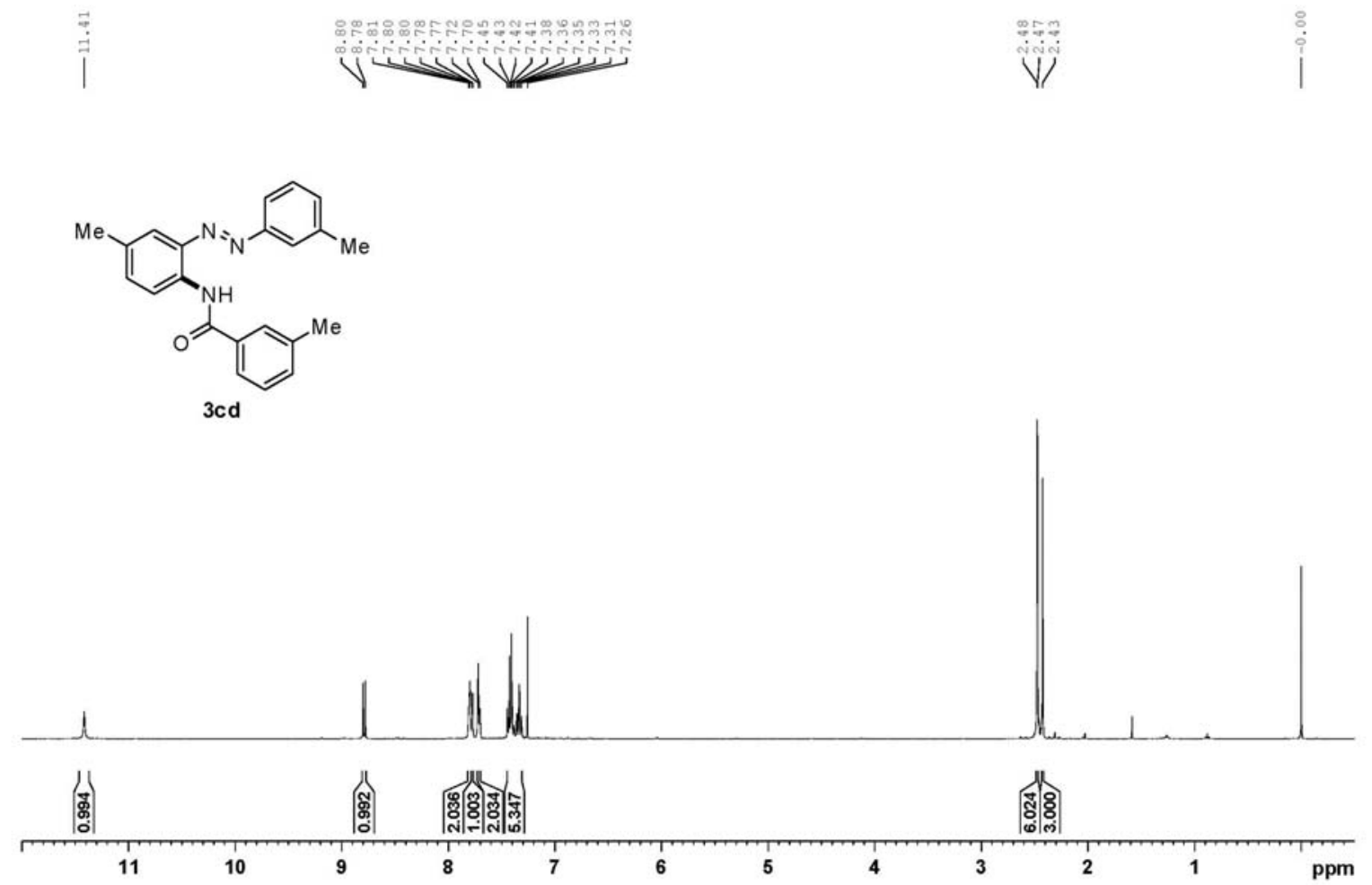

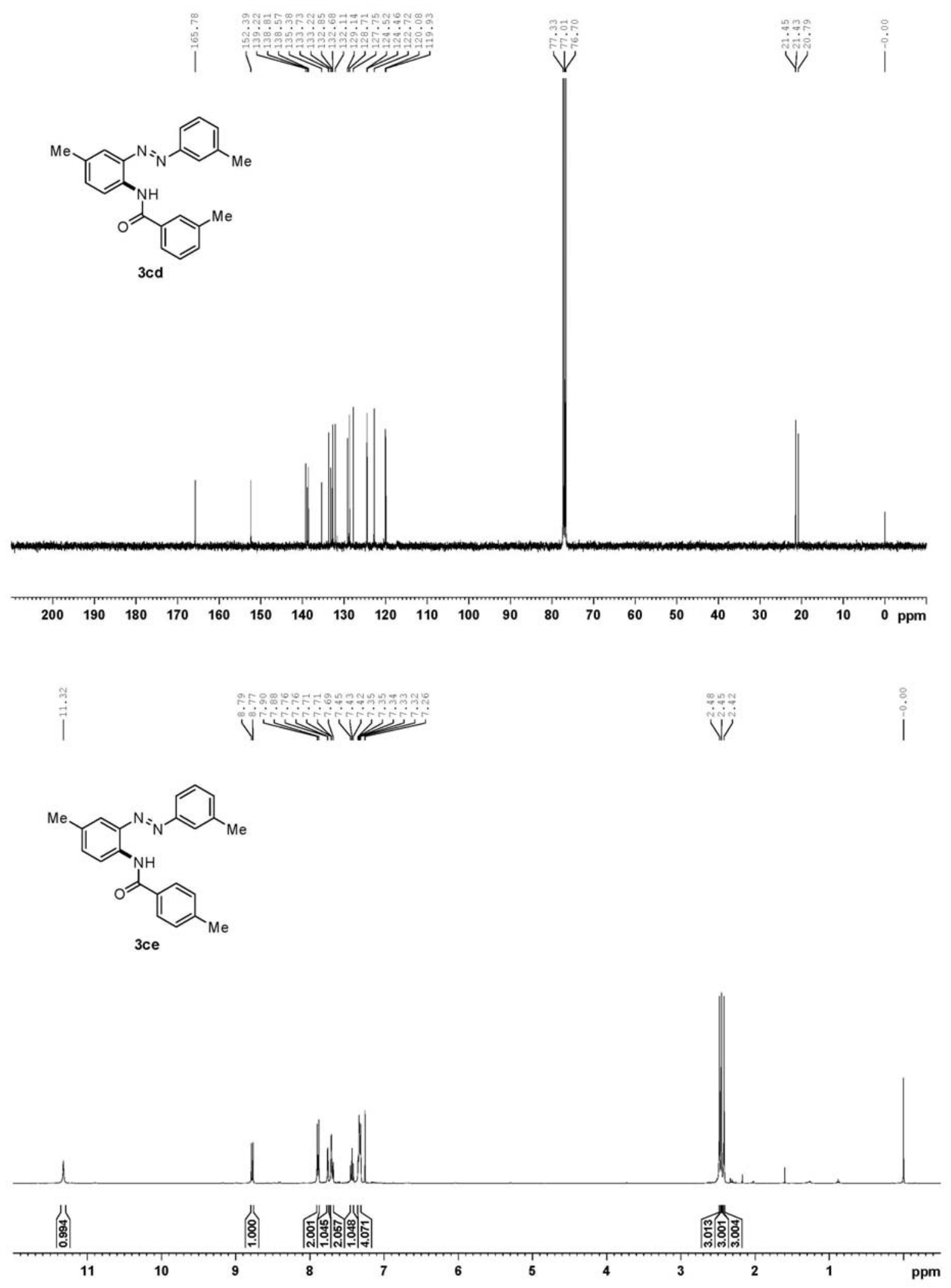

S48 


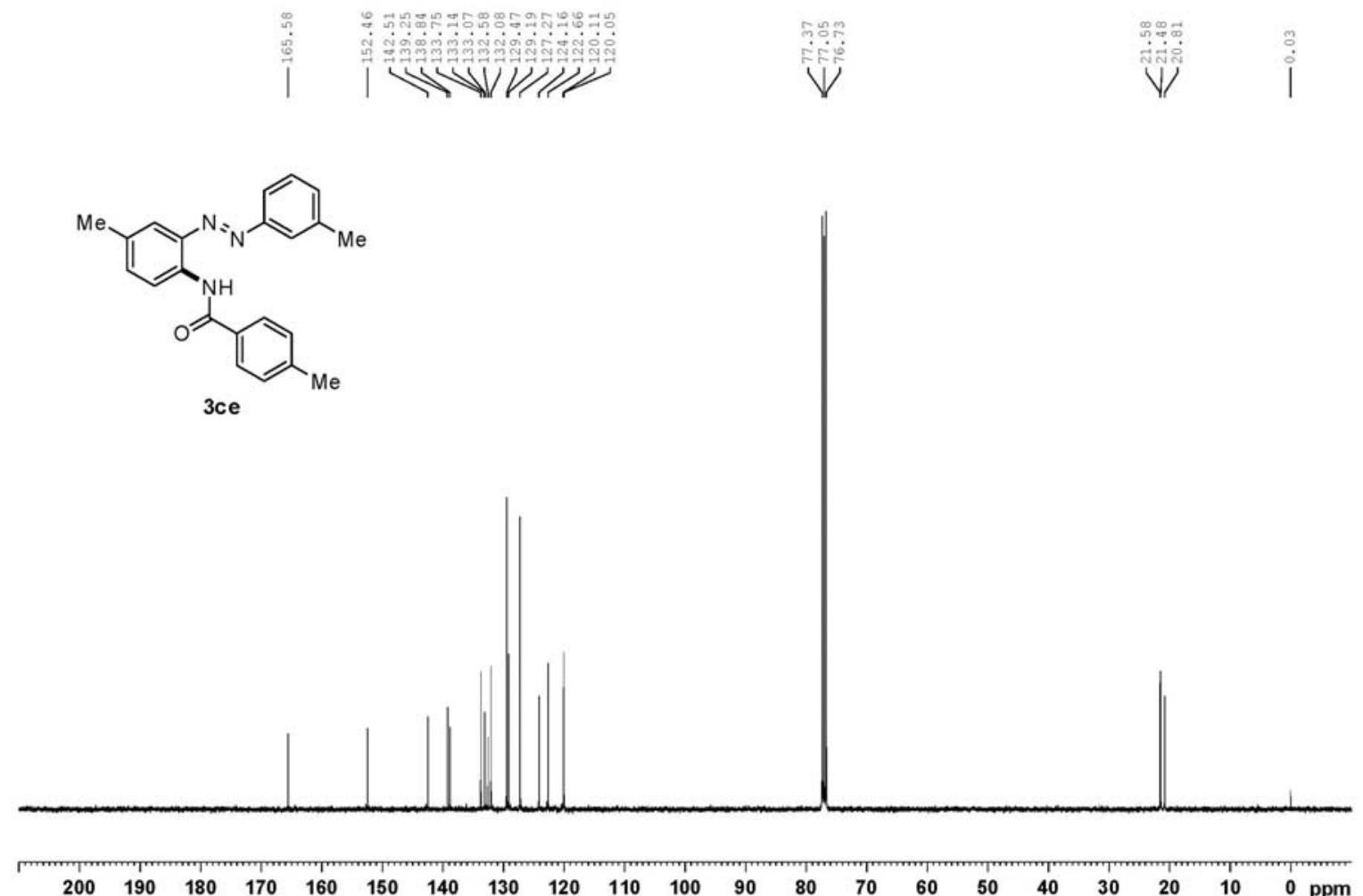

$\begin{array}{llllllllllllllllllllll}200 & 190 & 180 & 170 & 160 & 150 & 140 & 130 & 120 & 110 & 100 & 90 & 80 & 70 & 60 & 50 & 40 & 30 & 20 & 10 & 0 & \mathrm{ppm}\end{array}$

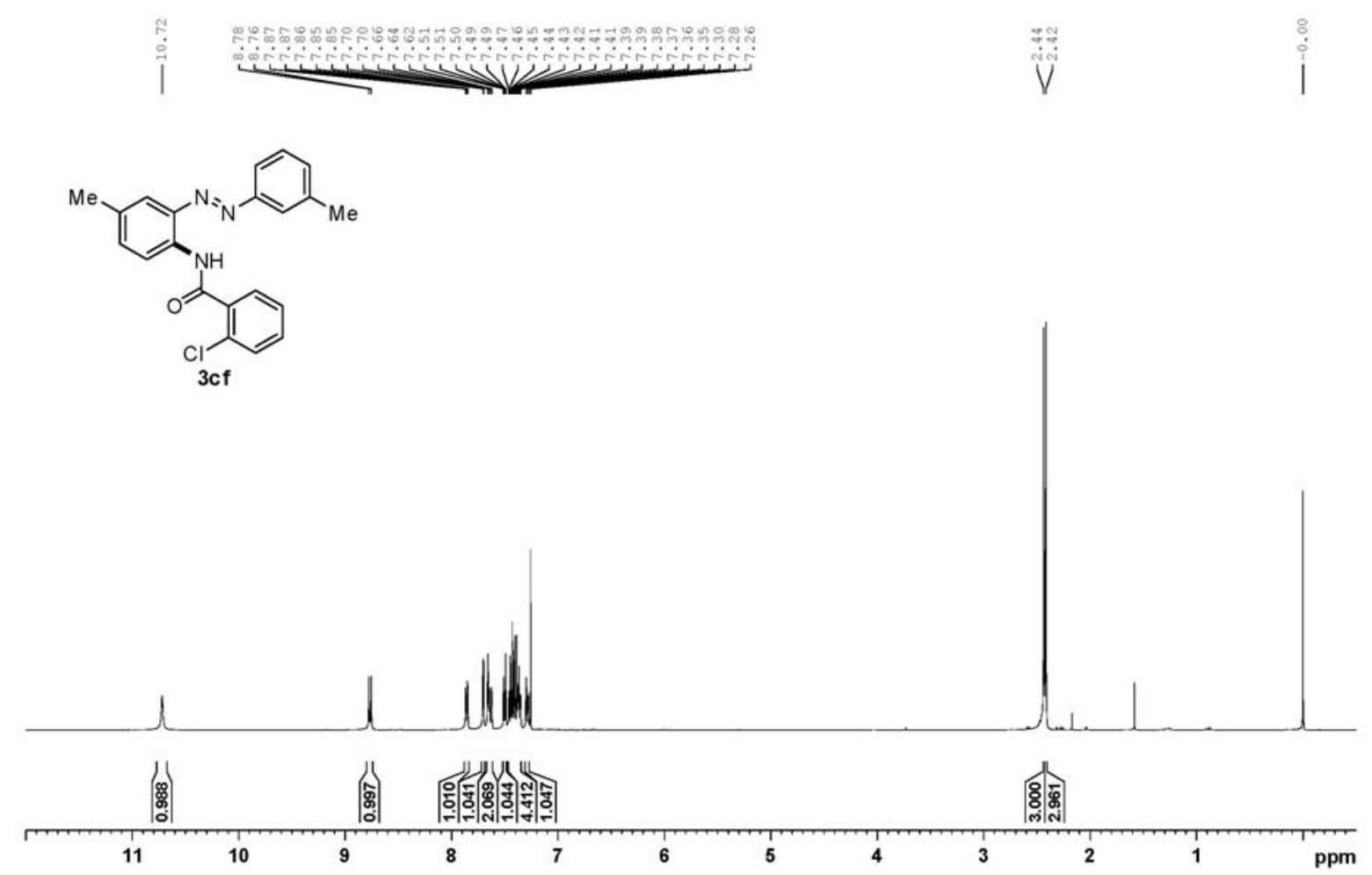




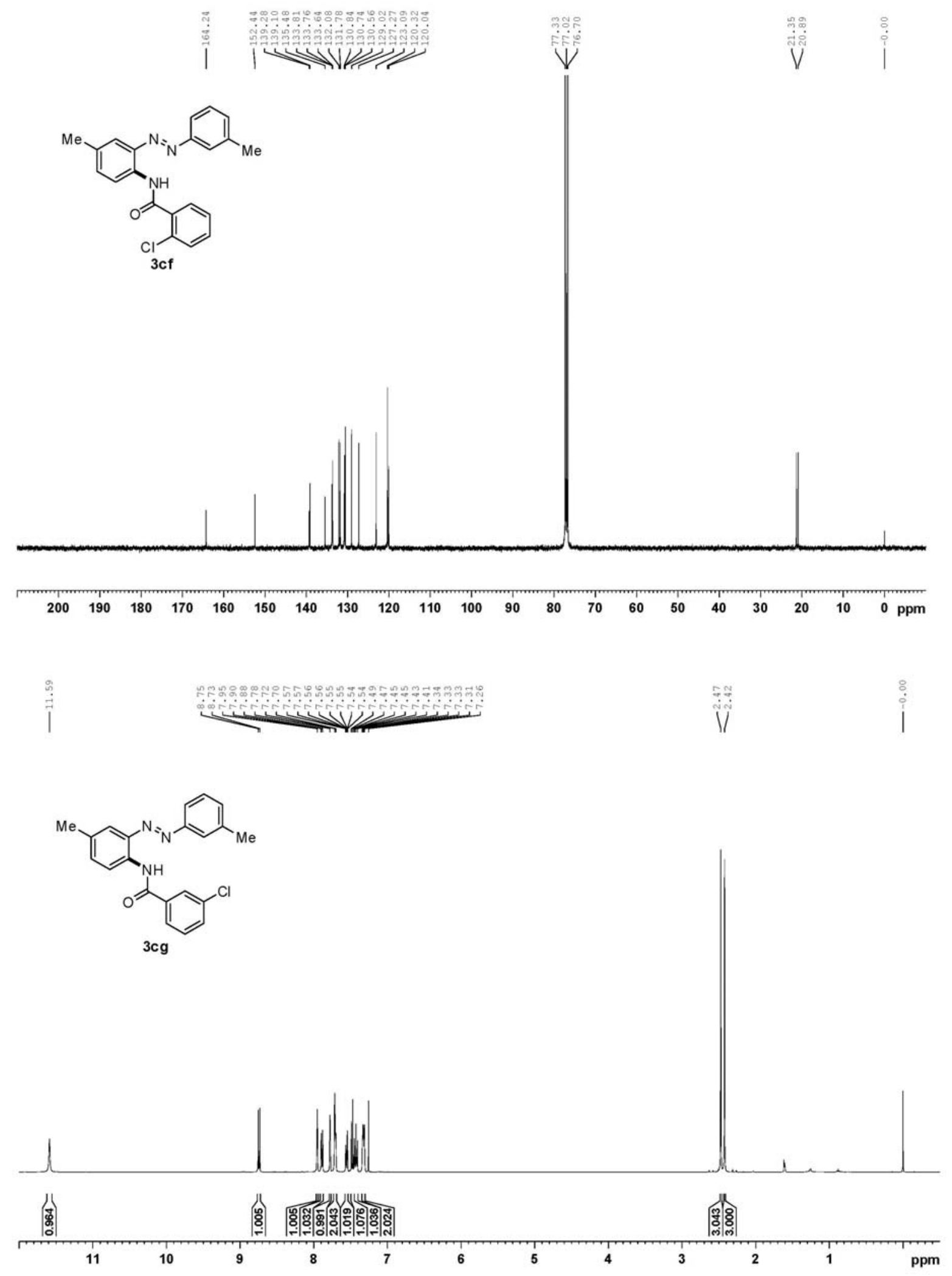




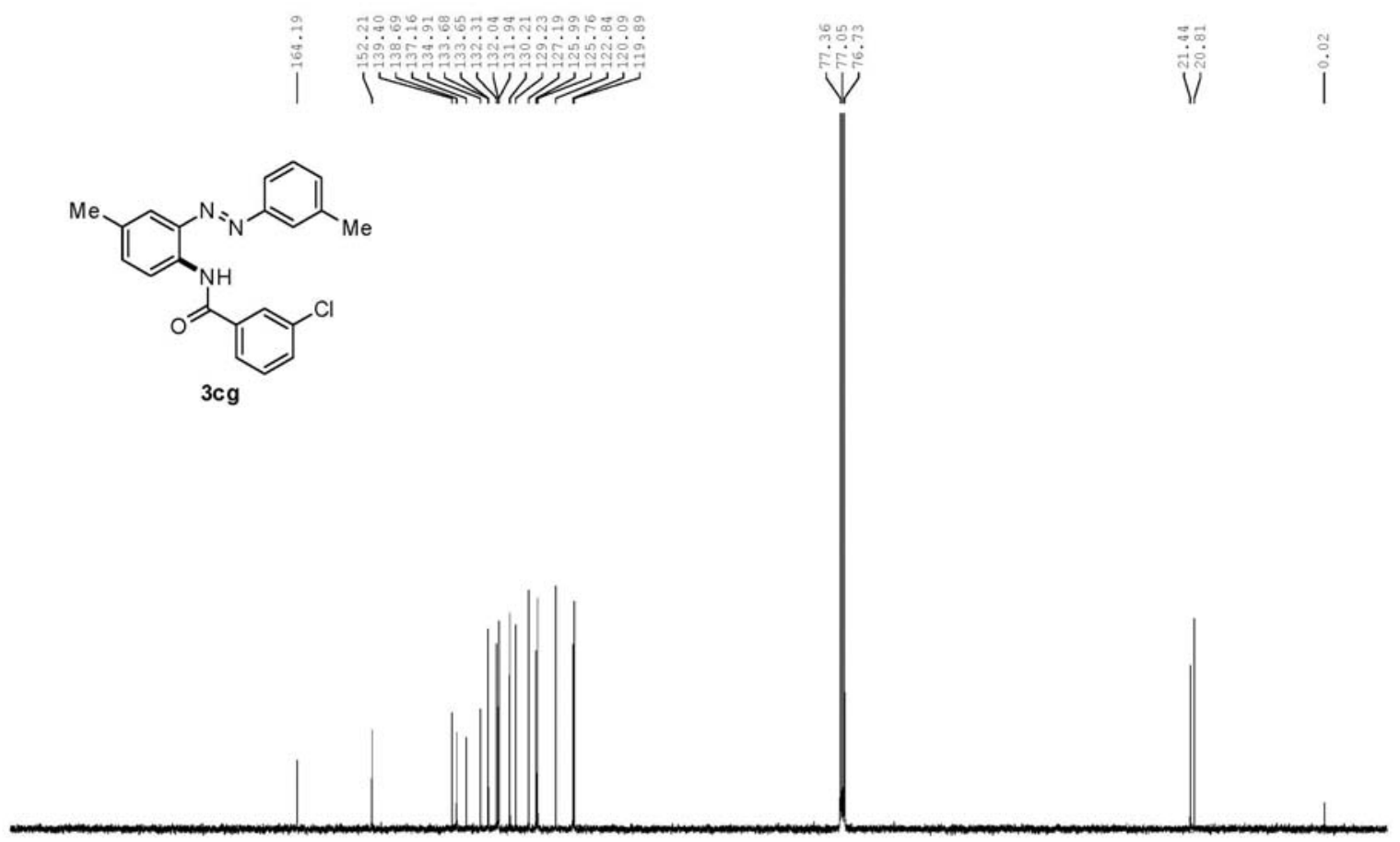

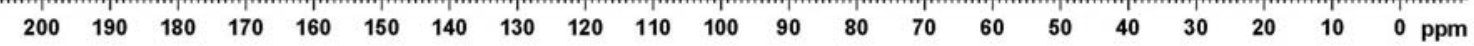
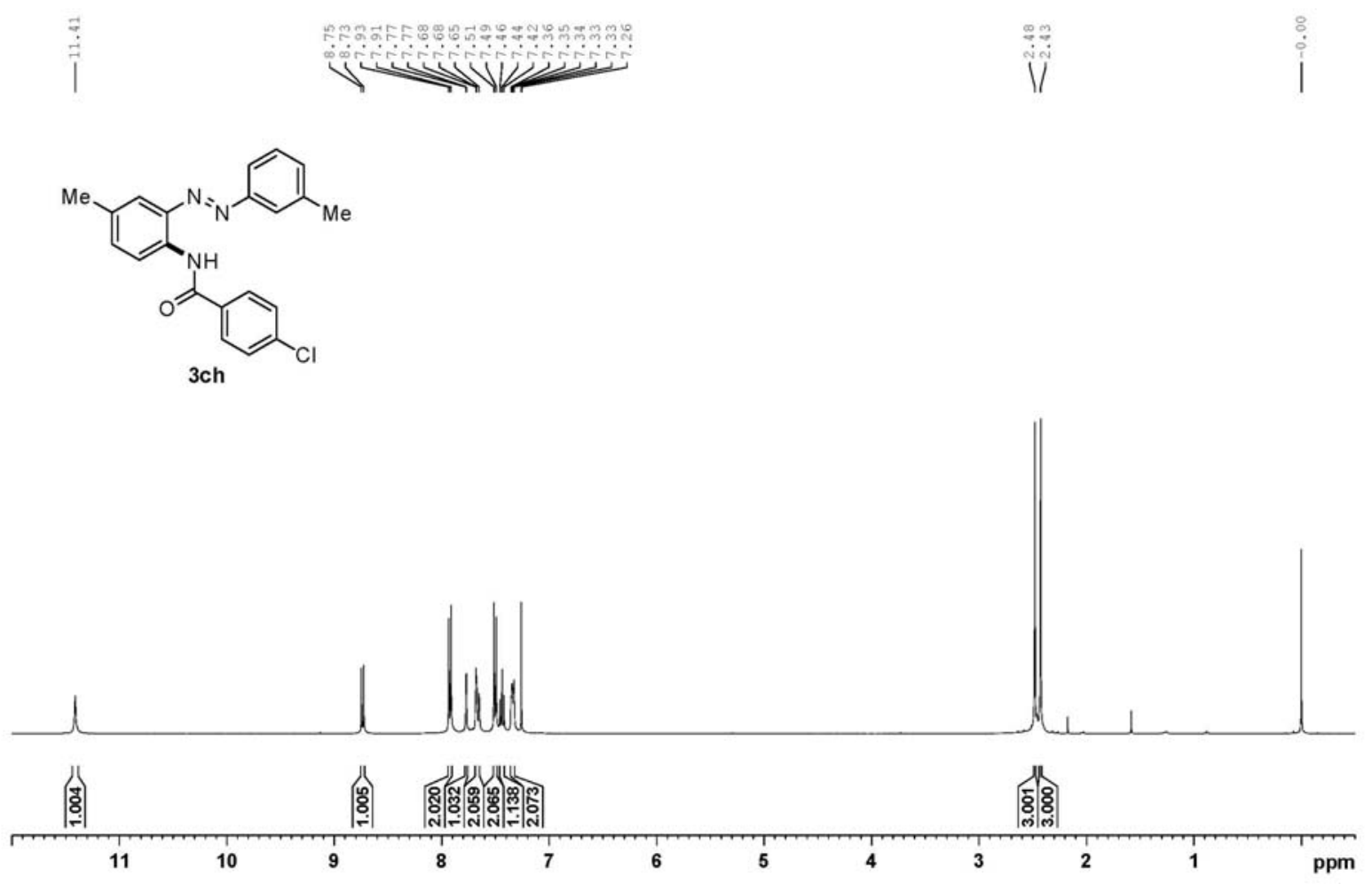

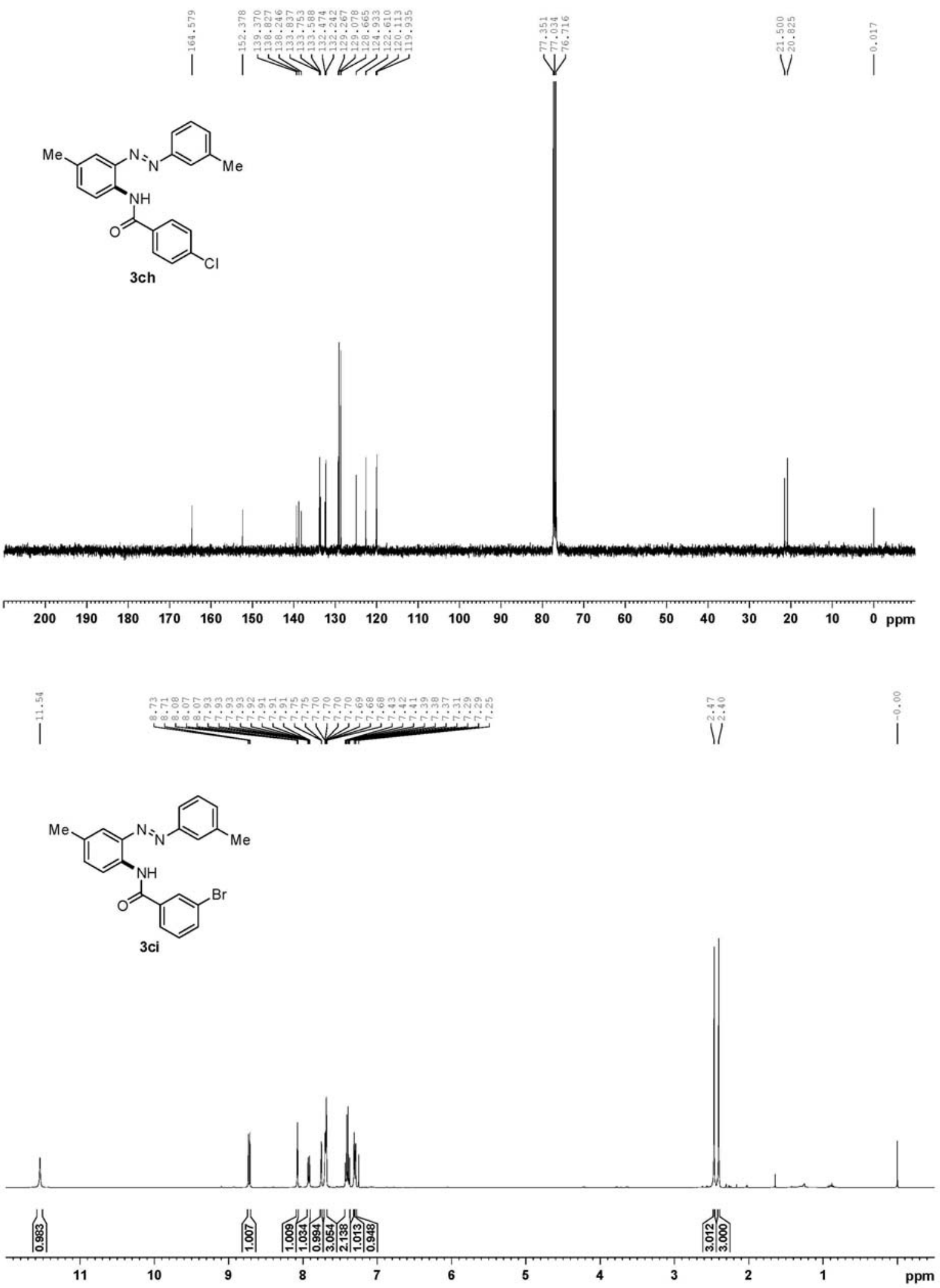


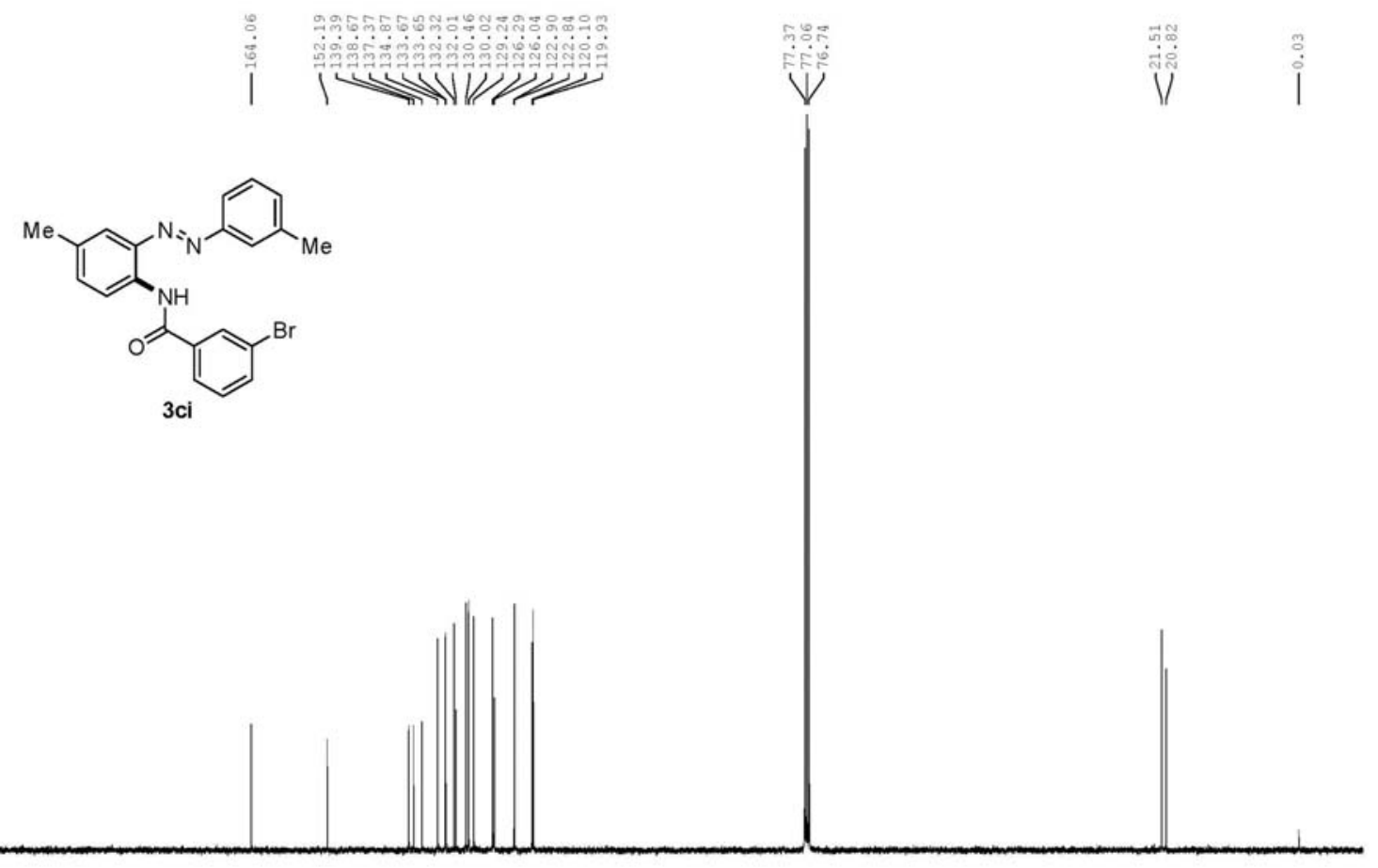

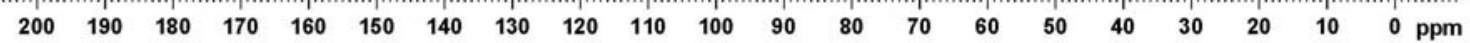
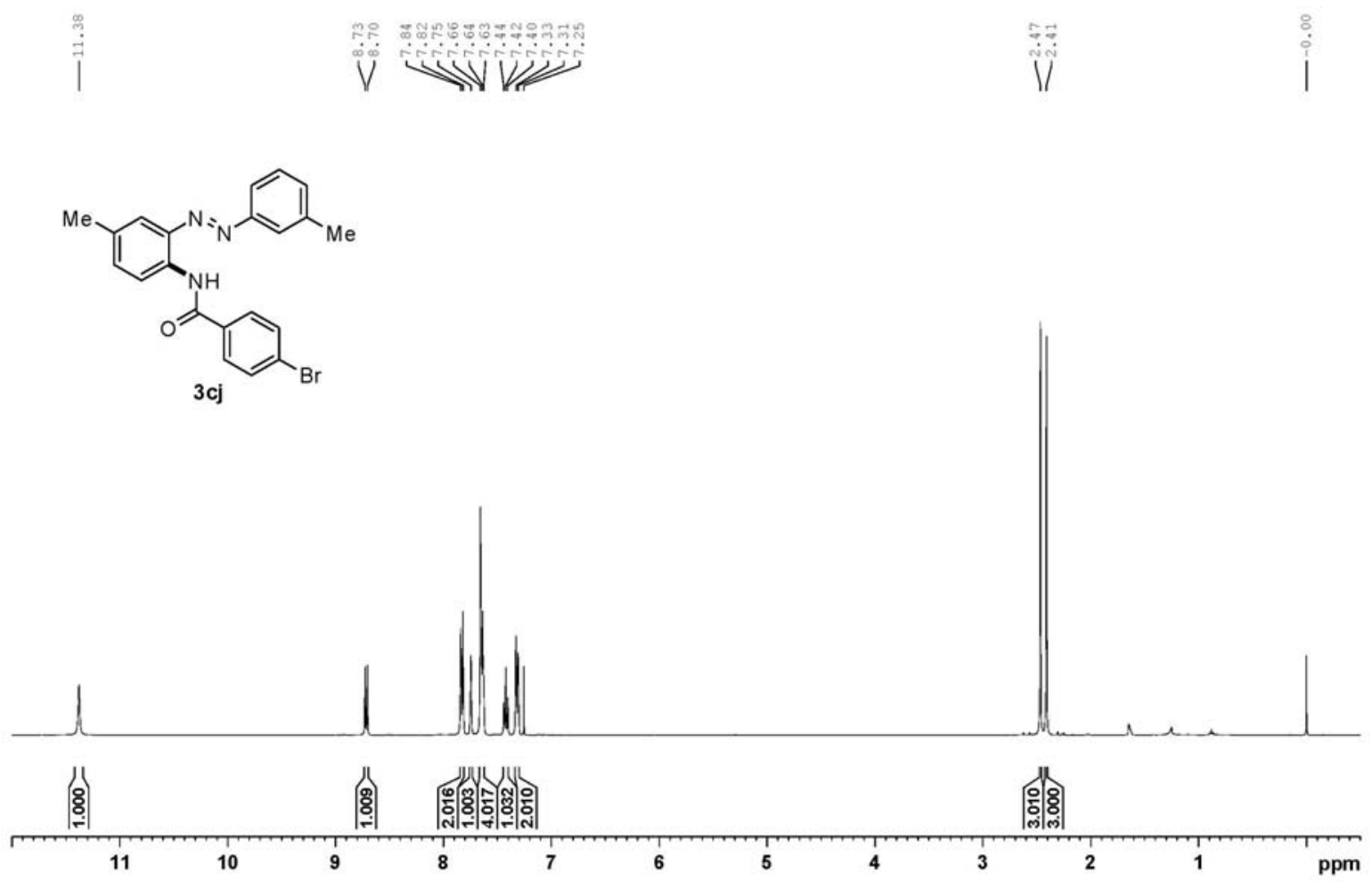

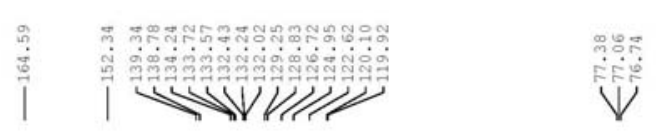

I
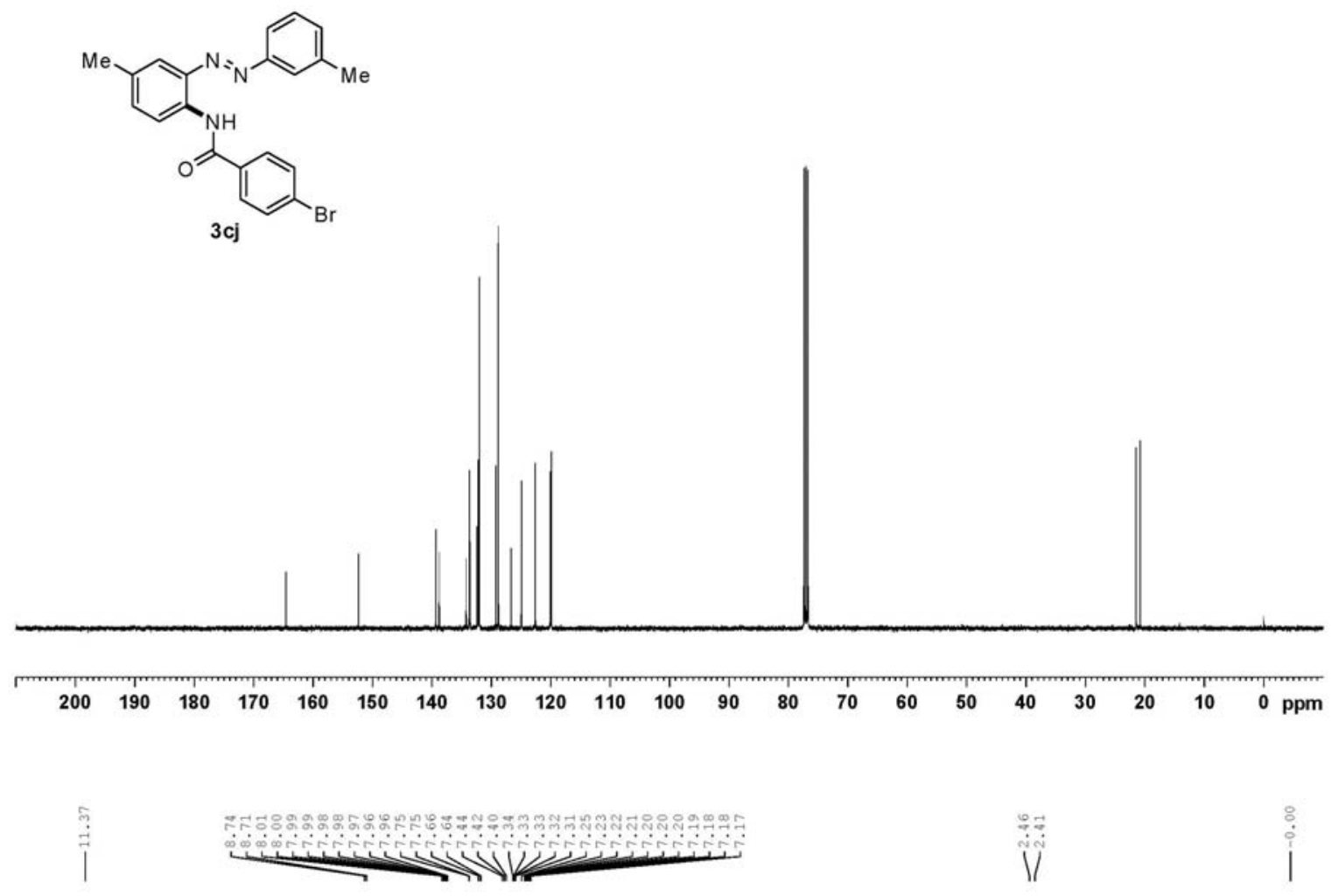

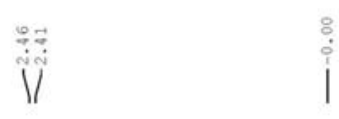
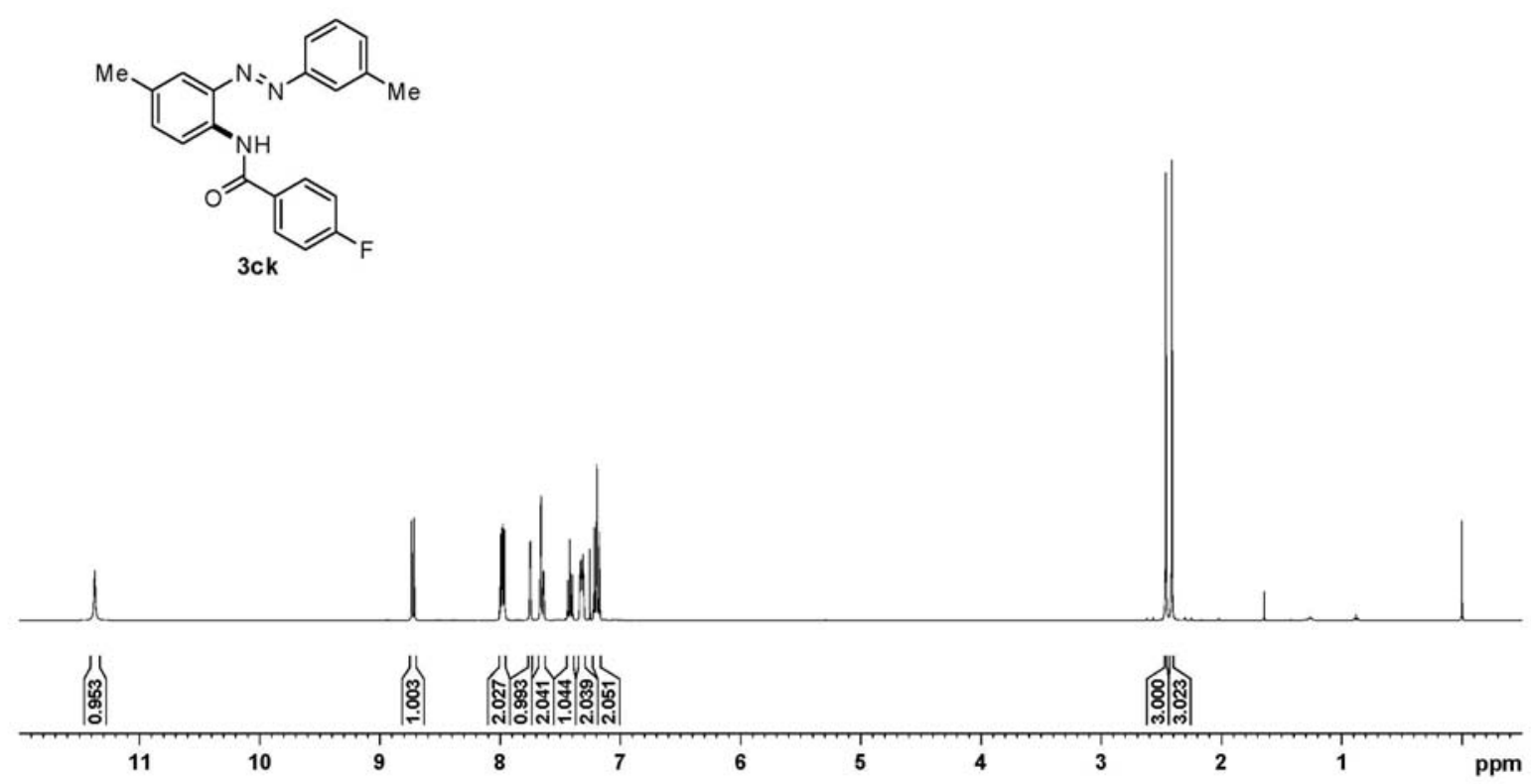

S54 


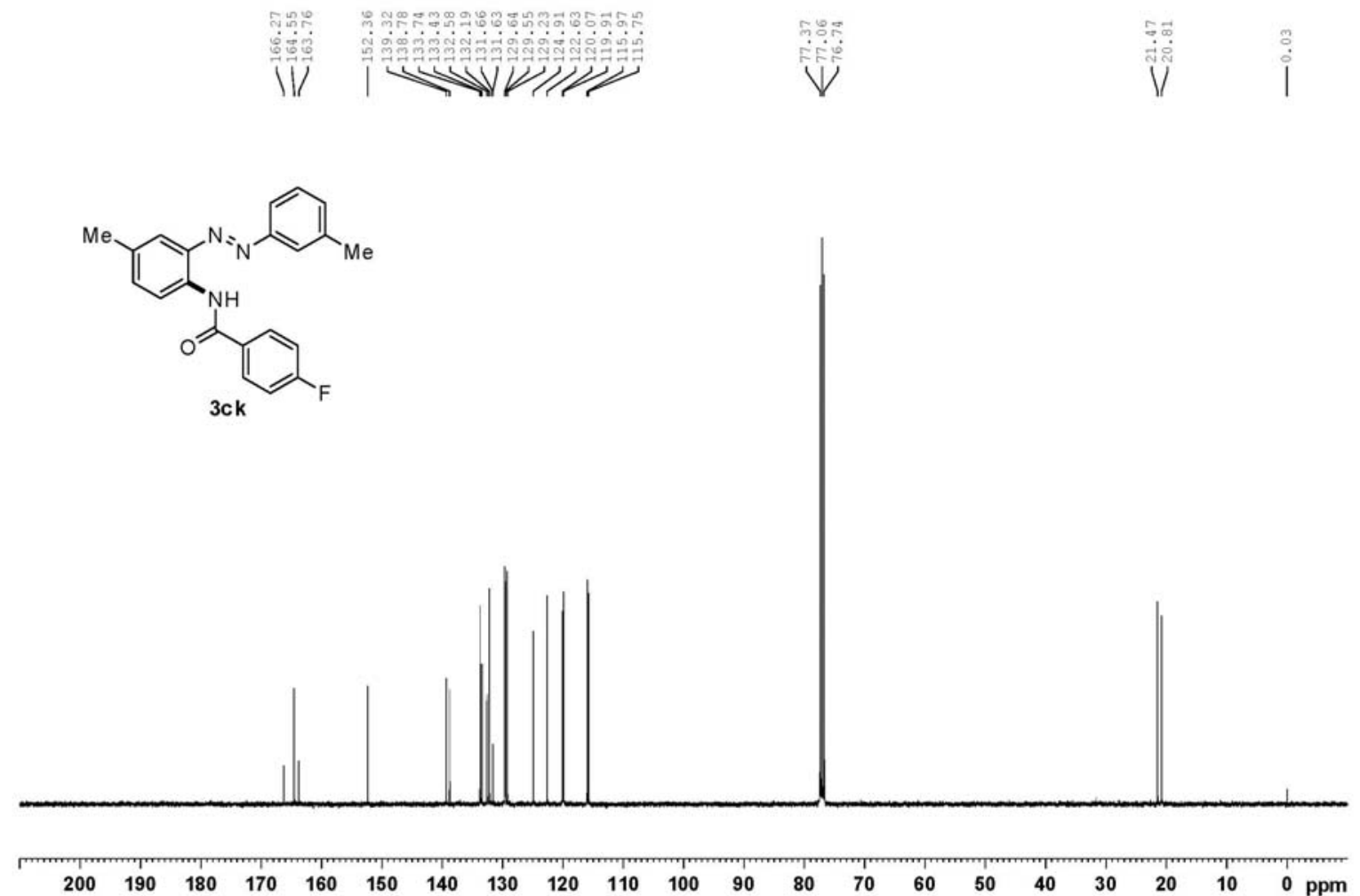

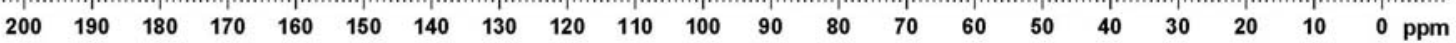<smiles>Cc1cccc(/N=N/c2cccc(C)c2)c1</smiles>

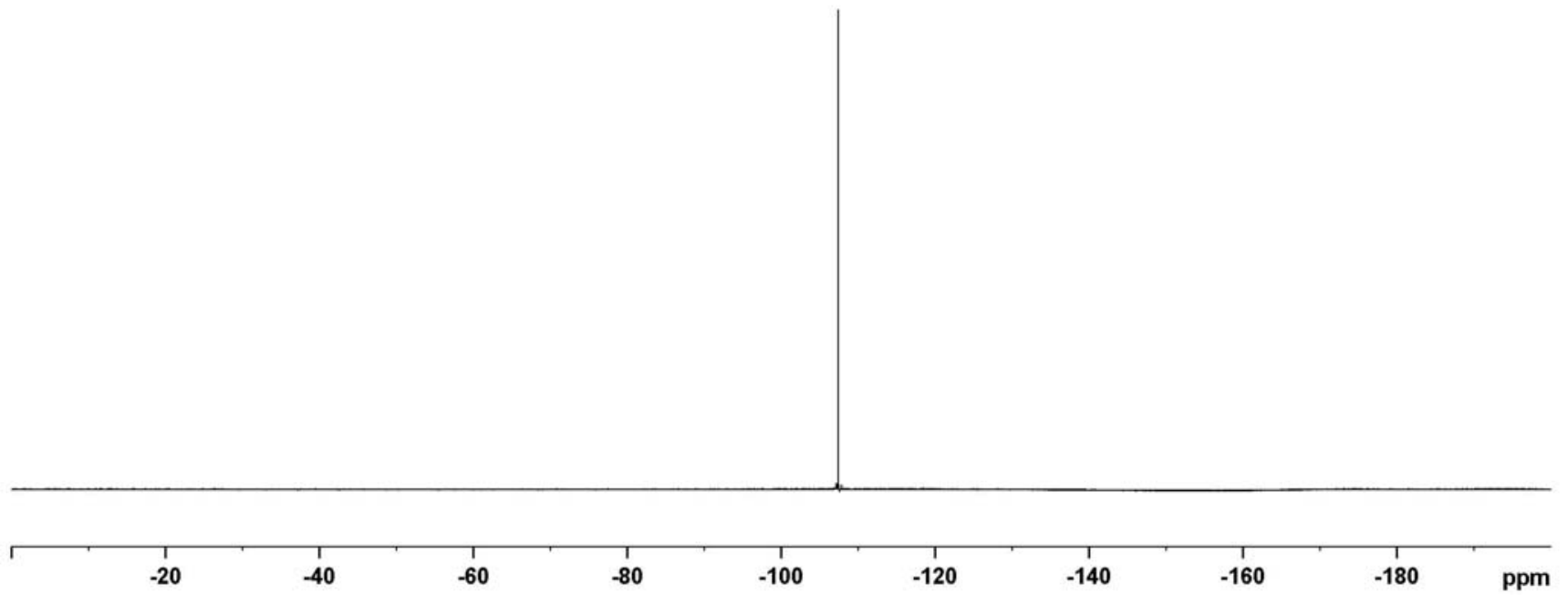


$\stackrel{\stackrel{\leftrightarrow}{\mid}}{\mid}$

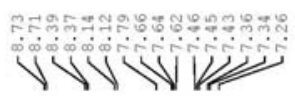

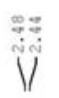

$\stackrel{\circ}{\grave{0}}$
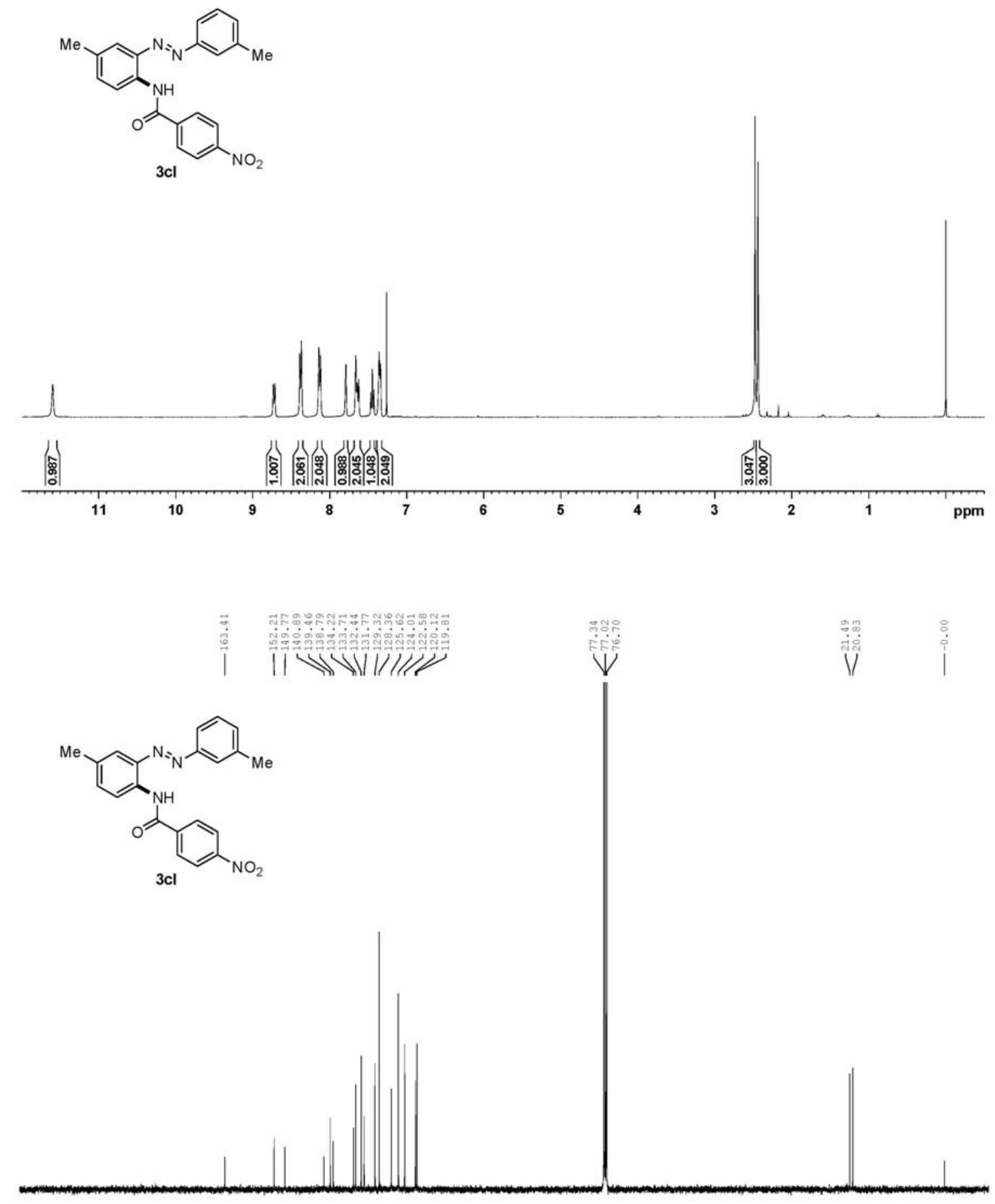

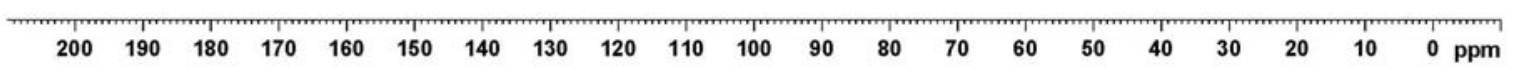

S56 
$\stackrel{\dddot{2}}{1}$

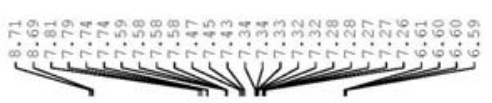

il

$\stackrel{\circ}{\dot{0}}$
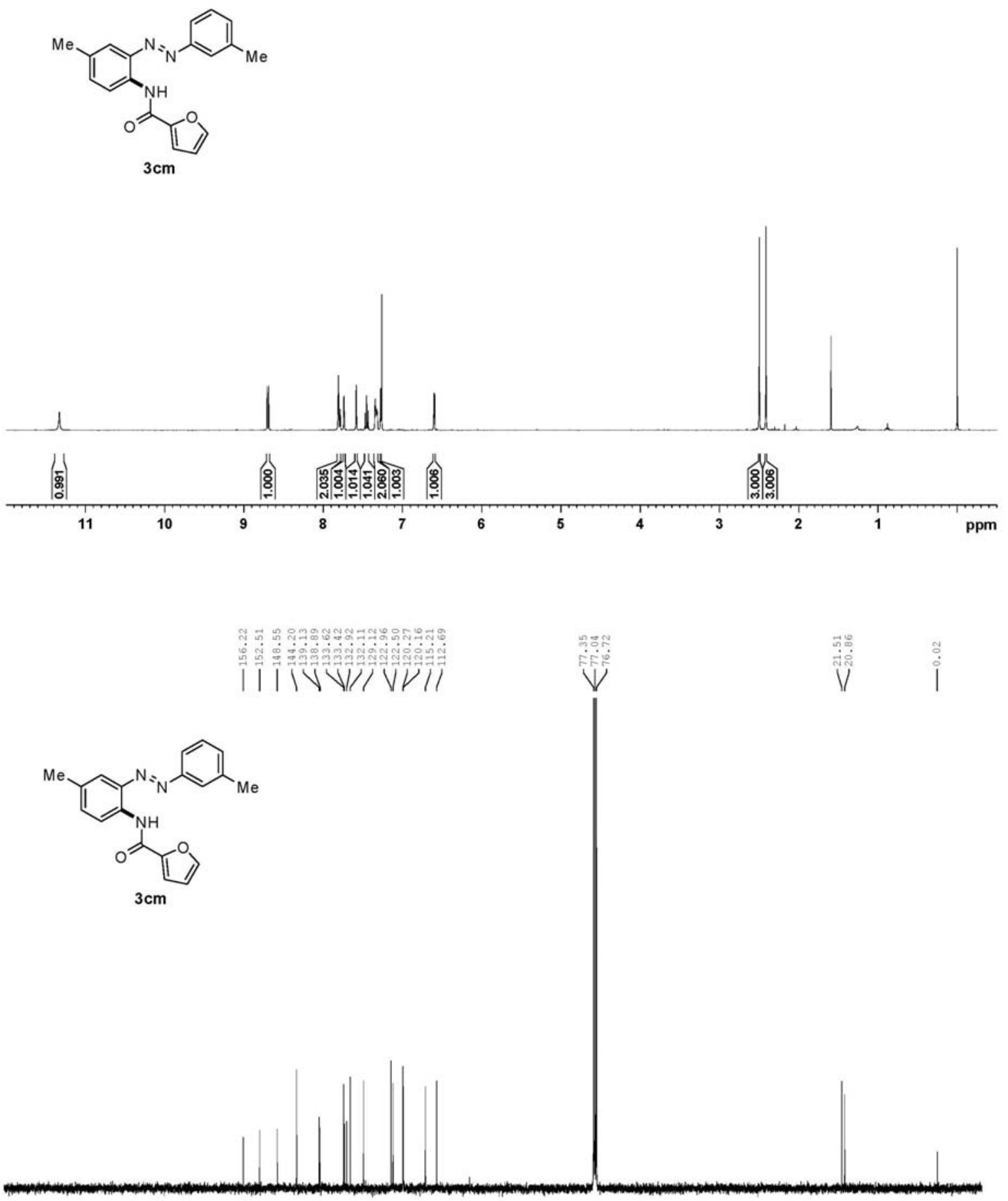

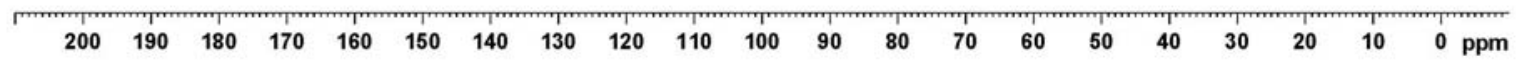

S57 


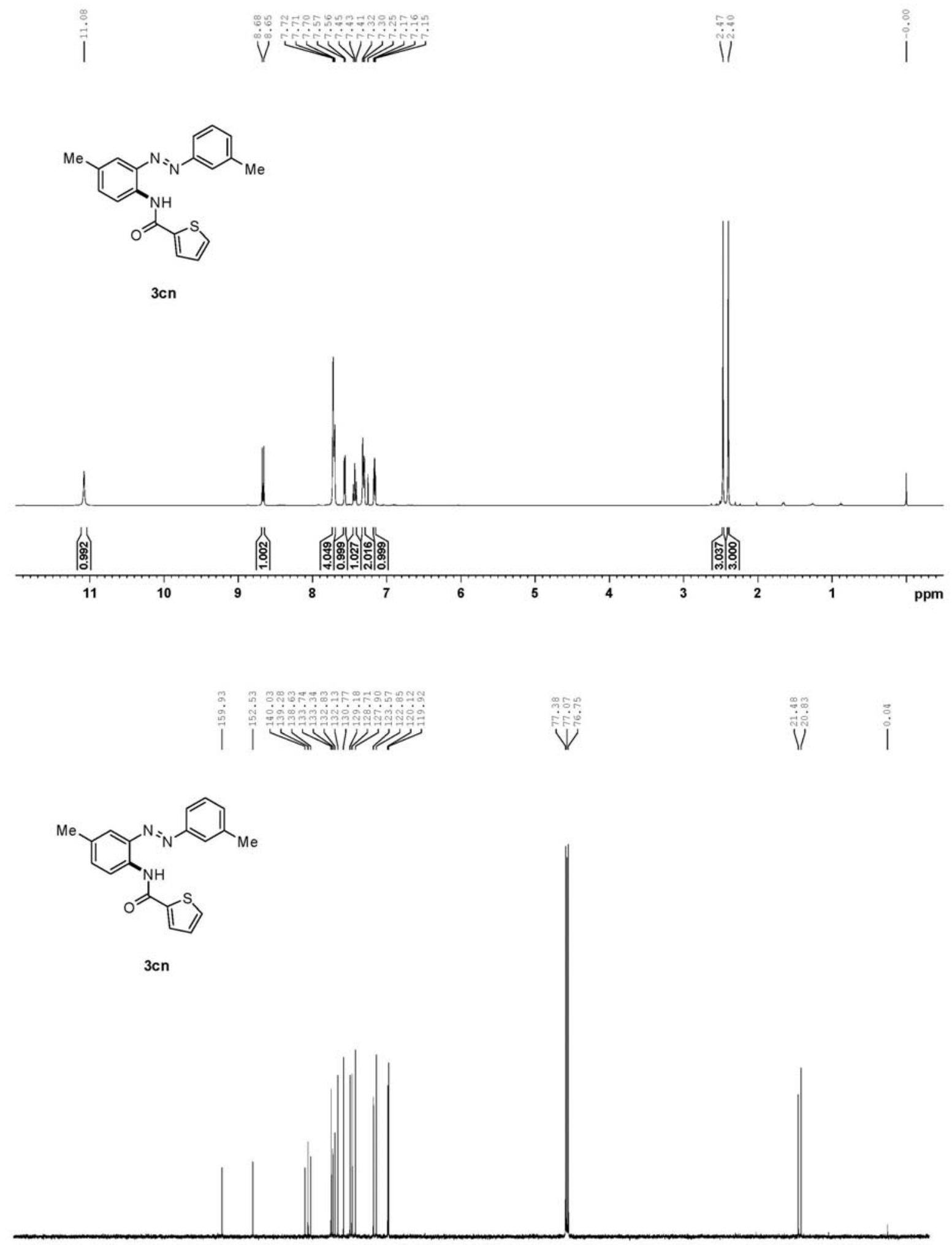

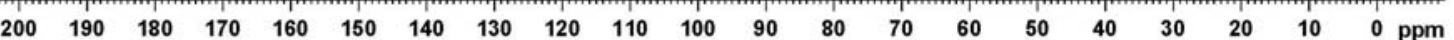




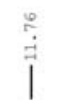

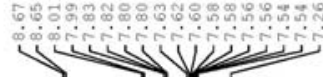

aaa
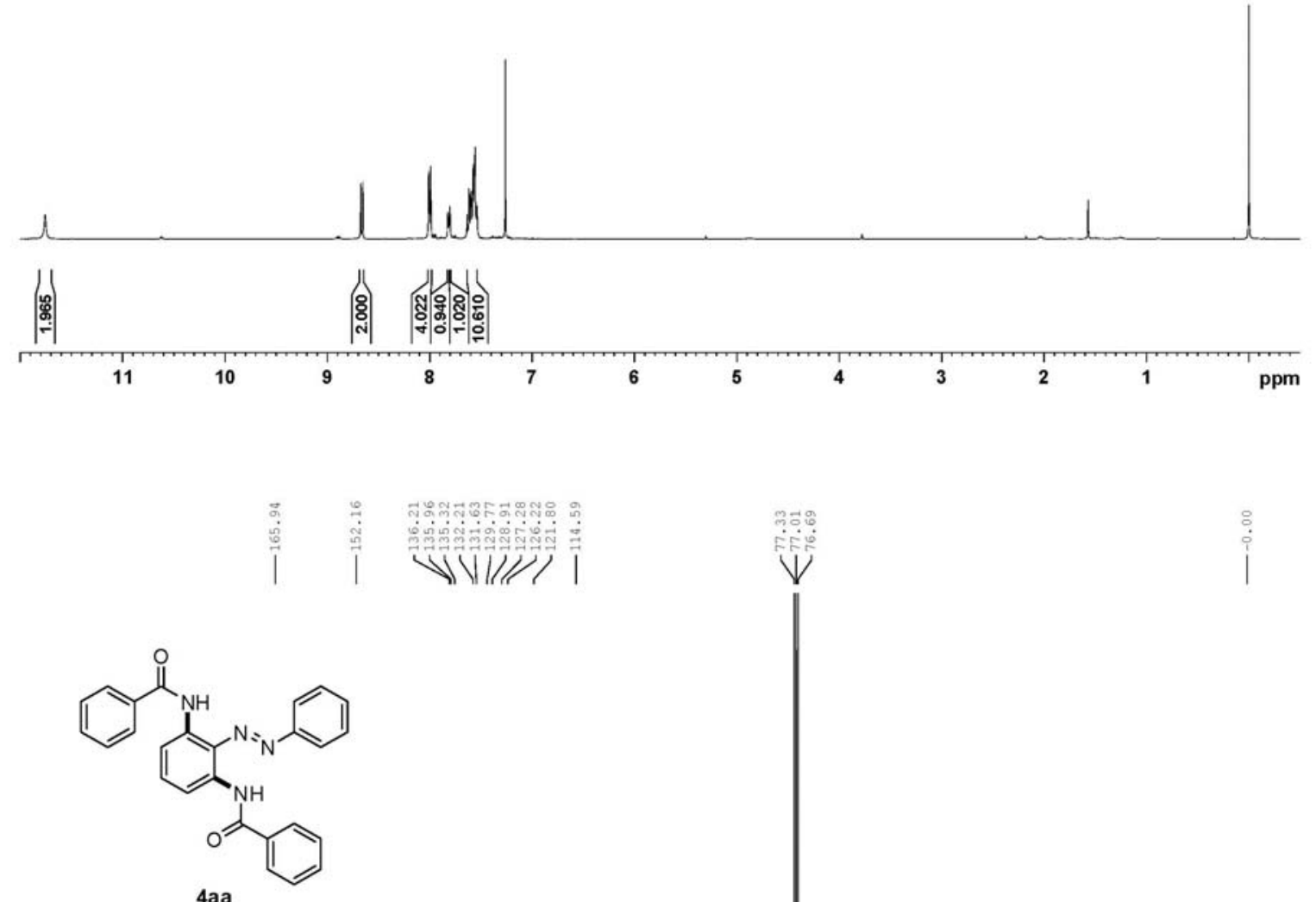

$4 a a$

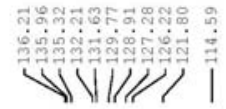

$\begin{array}{llllllllllllllllllllll}200 & 190 & 180 & 170 & 160 & 150 & 140 & 130 & 120 & 110 & 100 & 90 & 80 & 70 & 60 & 50 & 40 & 30 & 20 & 10 & 0 & \mathrm{ppm}\end{array}$ 

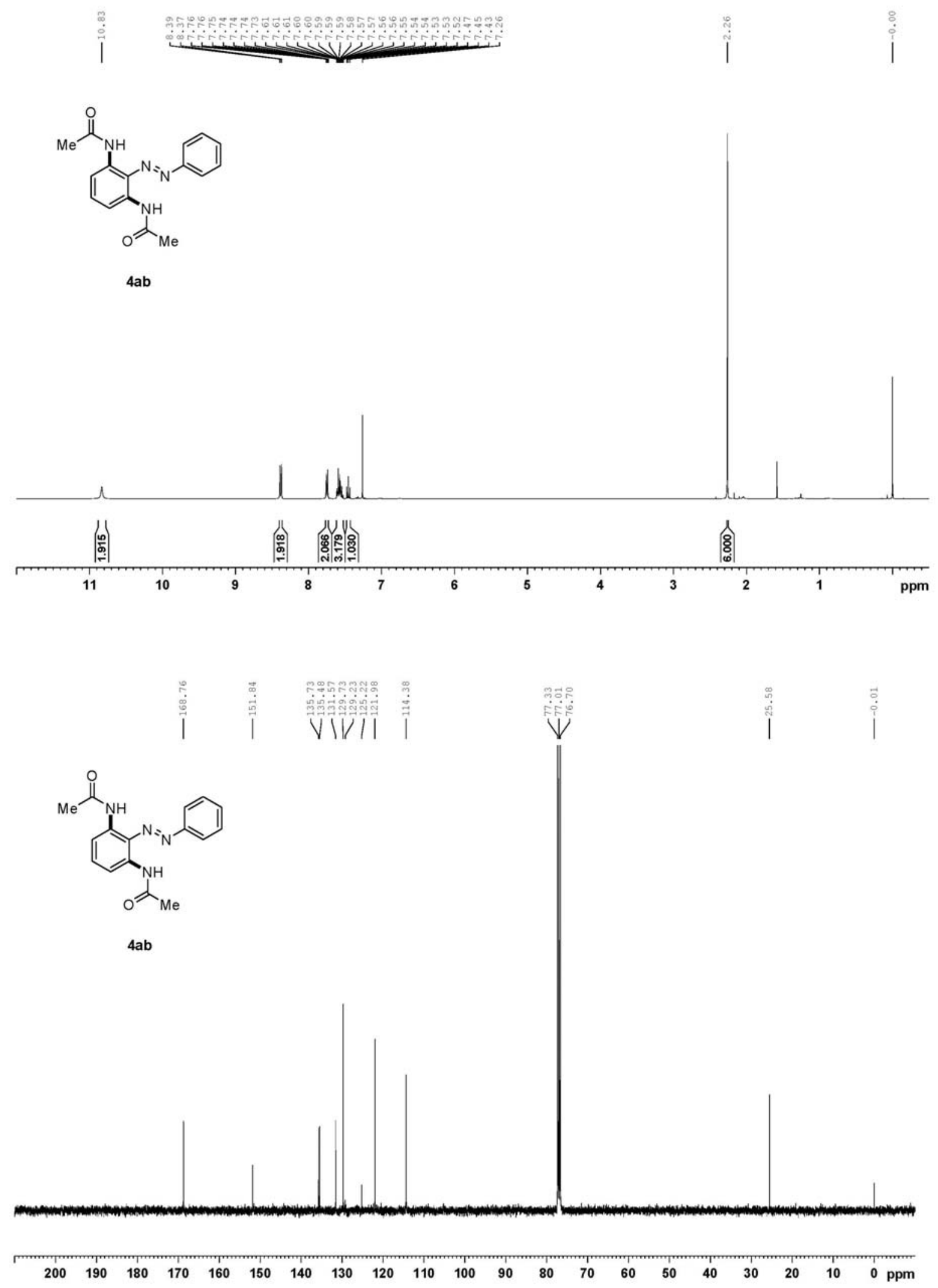

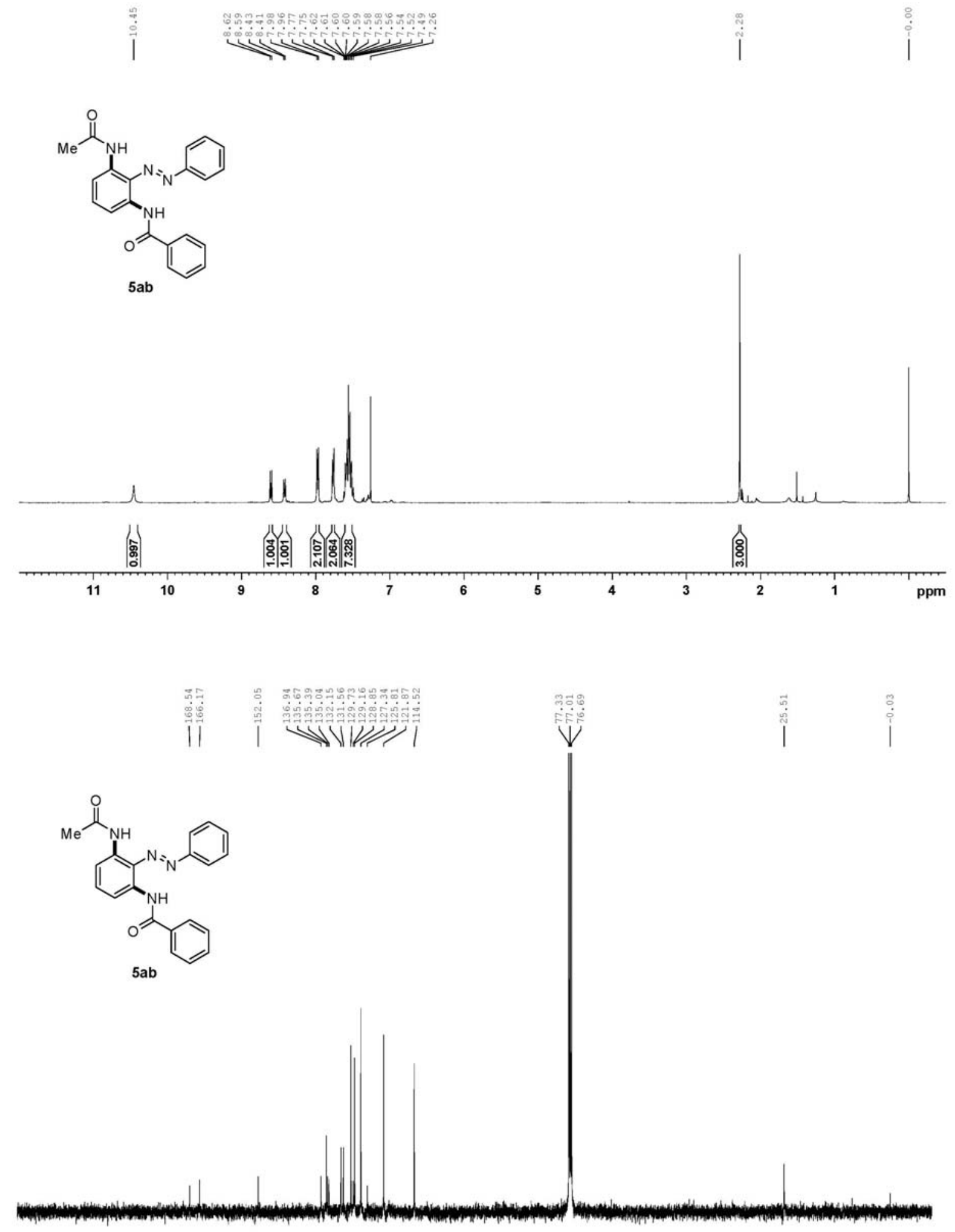

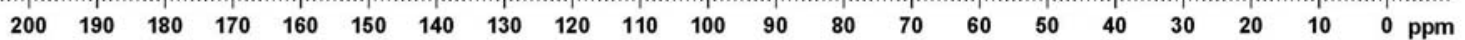



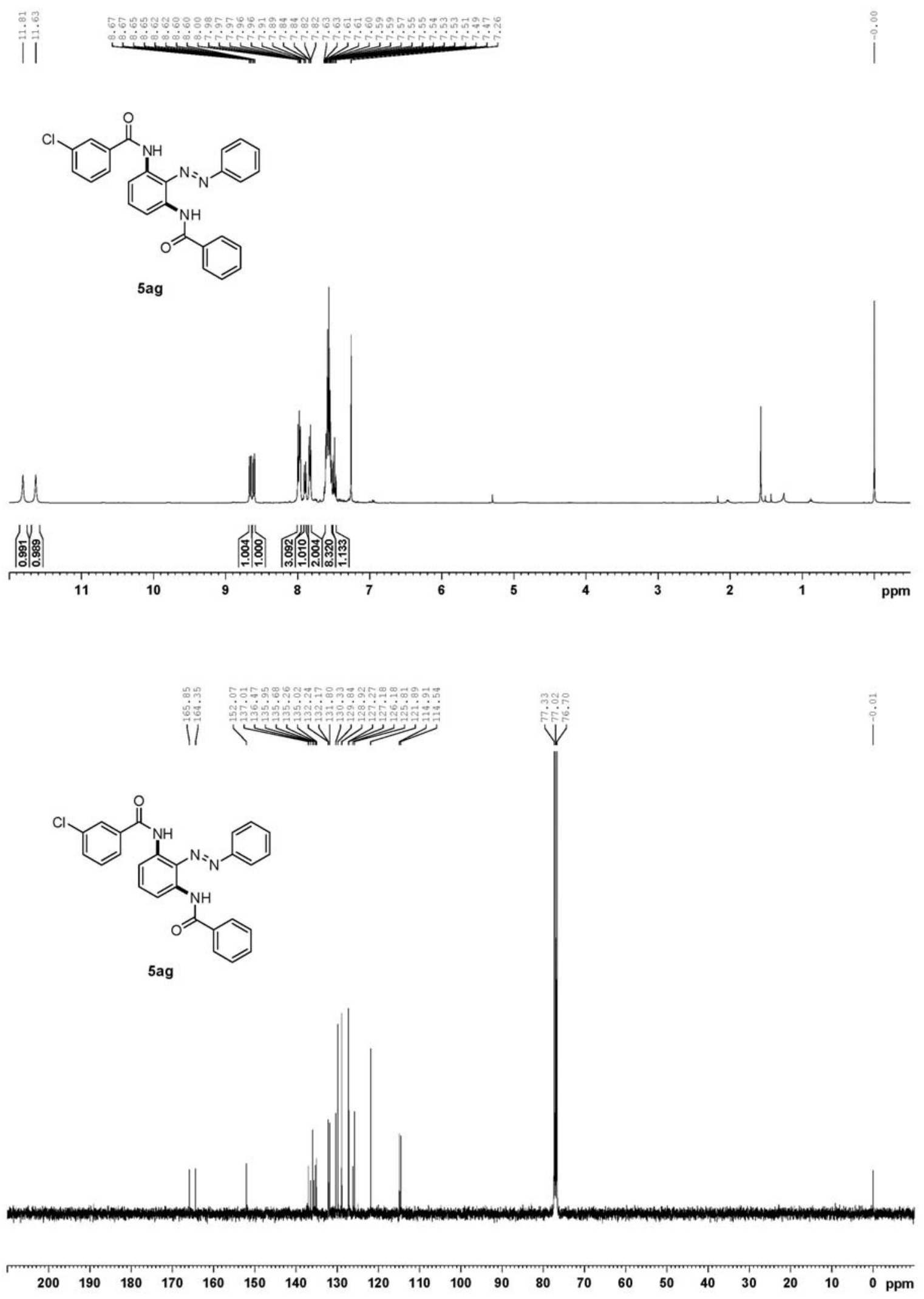
<smiles>O=C(Nc1cccc(NC(=O)c2ccc(Br)cc2)c1N=Nc1ccccc1)c1ccccc1</smiles>

5aj
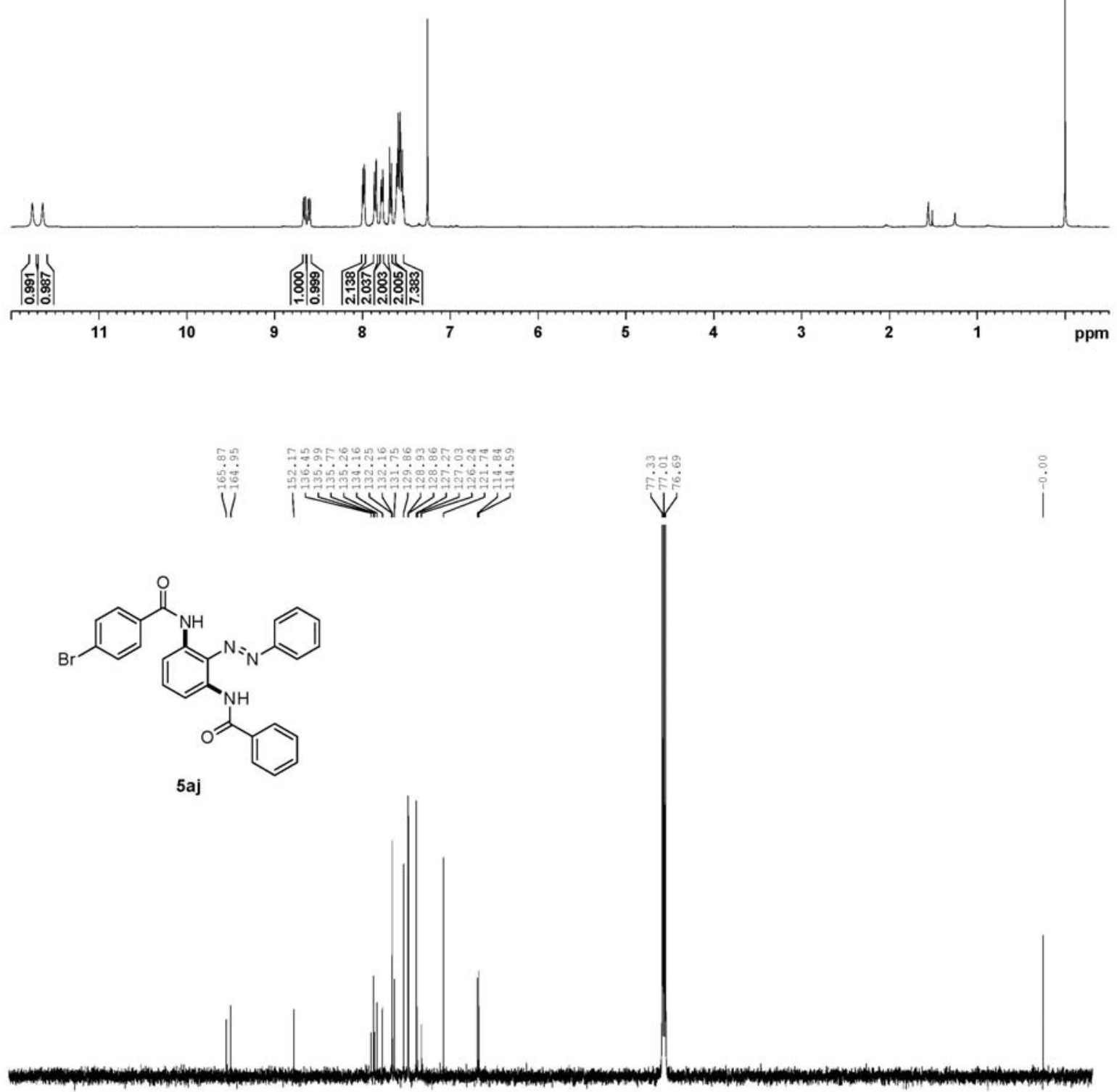

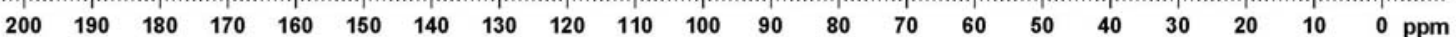


<smiles>O=C(Nc1cccc(NC(=O)c2cccs2)c1N=Nc1ccccc1)c1ccccc1</smiles>
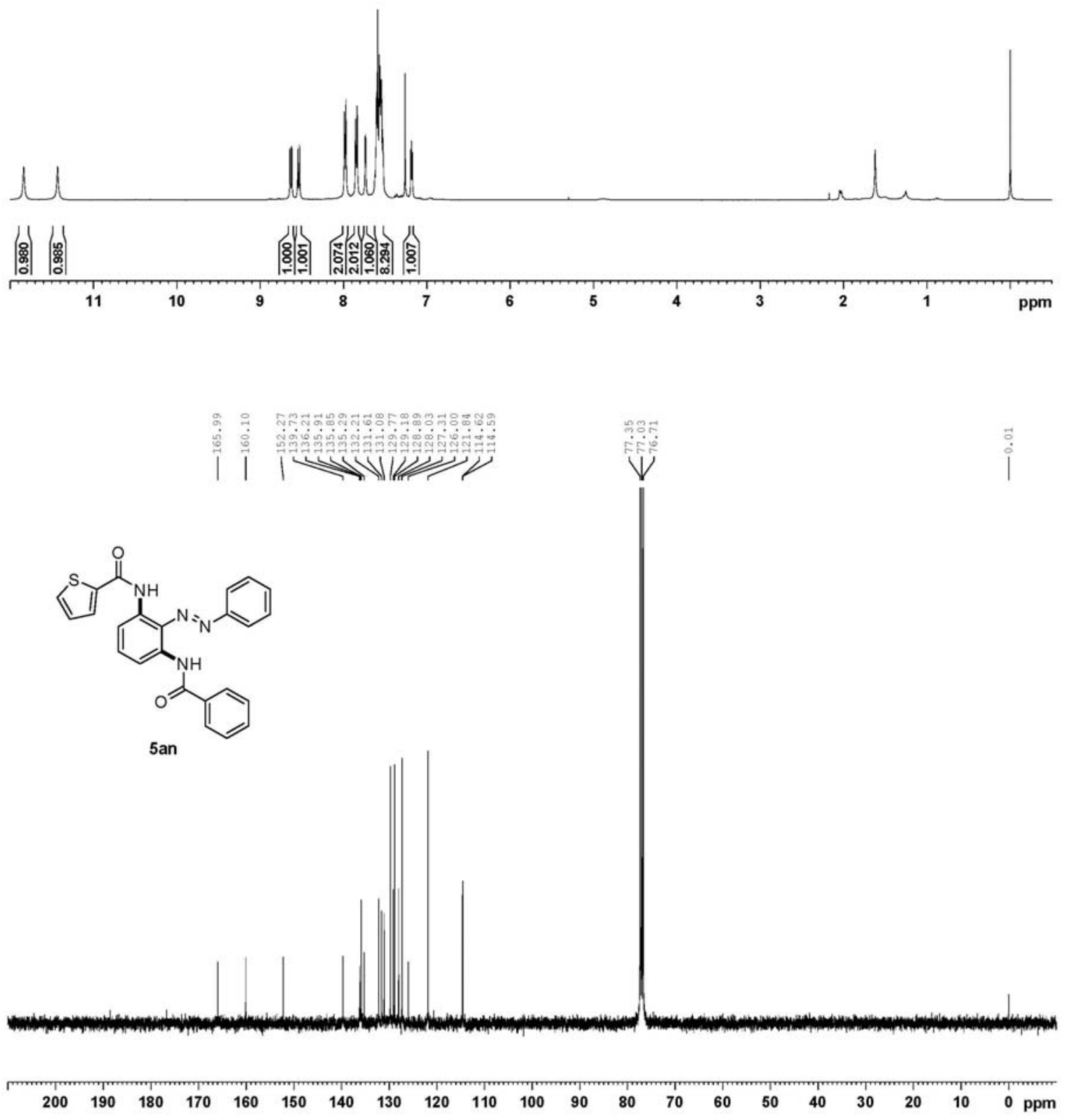


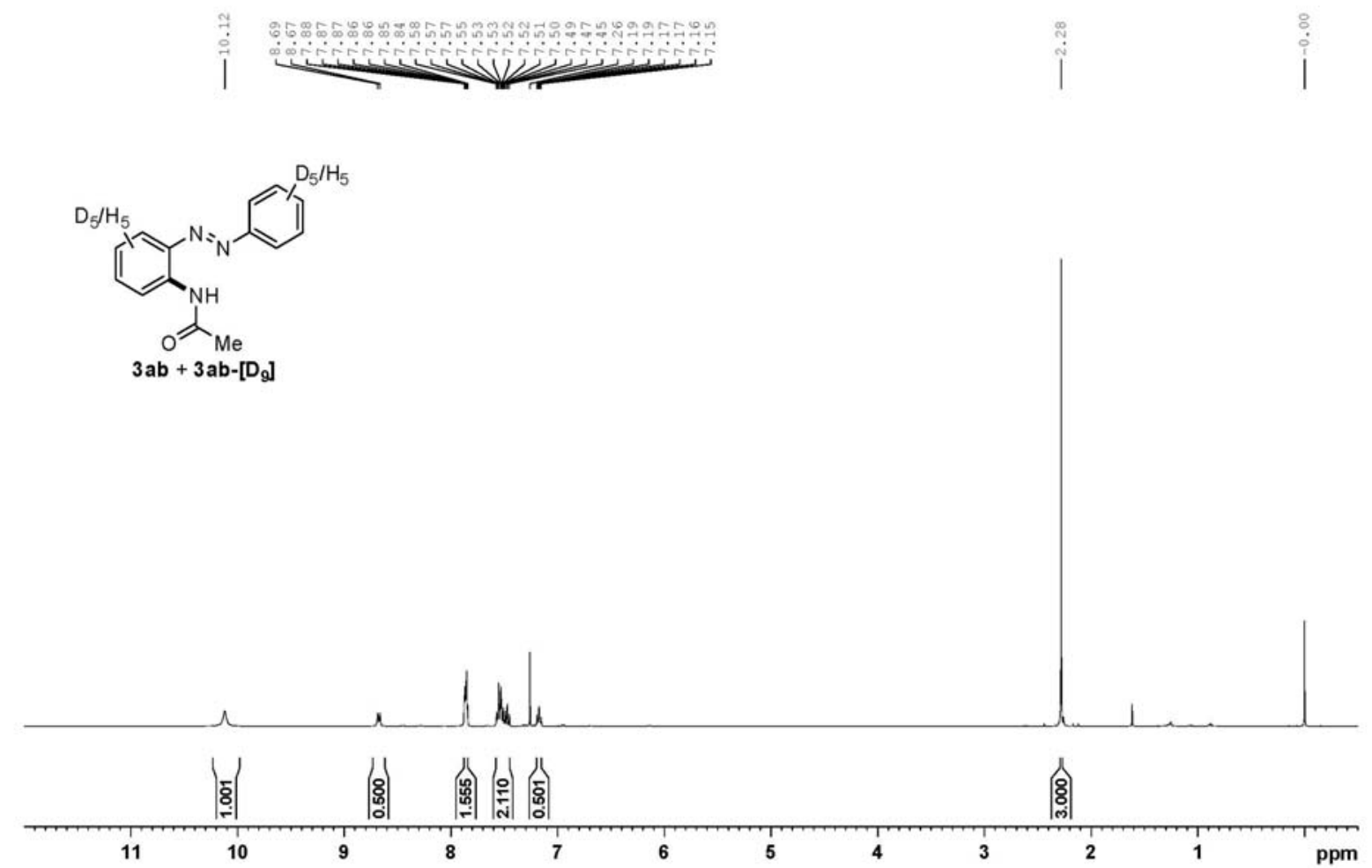

